\title{
Efficiency of Least Square and M-Estimators in Linear Regression with Dependent Errors
}

\author{
by \\ Zheng Yan
}

\begin{abstract}
A thesis submitted to the Faculty of Graduate Studies and Research in partial fulfillment of the requirements for the degree of Master of Statistics
\end{abstract}

Supervisor: Dr. M. Ould Haye

Ottawa-Carleton Institute of Mathematics and Statistics School of Mathematics and Statistics

Carleton University

Ottawa, ON 
Library and Archives Canada

Published Heritage Branch

395 Wellington Street Ottawa ON K1A ON4 Canada
Bibliotheqque et

Archives Canada

Direction du

Patrimoine de l'édition

395, rue Wellington

Ottawa ON K1A ON4

Canada
Your file Votre référence

ISBN: 978-0-494-68637-9

Our file Notre référence

ISBN: 978-0-494-68637-9

\section{NOTICE:}

The author has granted a nonexclusive license allowing Library and Archives Canada to reproduce, publish, archive, preserve, conserve, communicate to the public by telecommunication or on the Internet, loan, distribute and sell theses worldwide, for commercial or noncommercial purposes, in microform, paper, electronic and/or any other formats.

The author retains copyright ownership and moral rights in this thesis. Neither the thesis nor substantial extracts from it may be printed or otherwise reproduced without the author's permission.
AVIS:

L'auteur a accordé une licence non exclusive permettant à la Bibliothèque et Archives Canada de reproduire, publier, archiver, sauvegarder, conserver, transmettre au public par télécommunication ou par l'Internet, prêter, distribuer et vendre des thèses partout dans le monde, à des fins commerciales ou autres, sur support microforme, papier, électronique et/ou autres formats.

L'auteur conserve la propriété du droit d'auteur et des droits moraux qui protège cette thèse. $\mathrm{Ni}$ la thèse ni des extraits substantiels de celle-ci ne doivent être imprimés ou autrement reproduits sans son autorisation.
In compliance with the Canadian Privacy Act some supporting forms may have been removed from this thesis.

While these forms may be included in the document page count, their removal does not represent any loss of content from the thesis.
Conformément à la loi canadienne sur la protection de la vie privée, quelques formulaires secondaires ont été enlevés de cette thèse.

Bien que ces formulaires aient inclus dans la pagination, il n'y aura aucun contenu manquant.

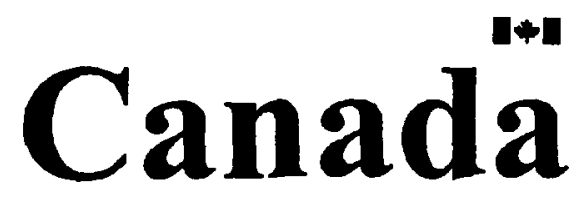




\section{Abstract}

We examine the asymptotic efficiency of the least square estimator (LSE), relative to the best linear unbiased estimator (BLUE) in linear regression with dependent errors. First, some definitions and notations are given. Next, we introduce LSE and BLUE in chapter 3. With dependent errors, we are more interested in LSE than BLUE, since the covariance matrix of the errors is seldom known. In chapter 4 , we find that the LSE $\hat{\beta}$ of the regression coefficients is asymptotically efficient relative to the BLUE if and only if the spectral density is constant on each of the elements of $S$ (regression spectrum). In addition, we study the asymptotic properties of M-estimators of regression parameters in linear models with dependent errors in chapter 5. We derive the Bahadur representations and establish a central limit theorem for LSE and BLUE. At last, we illustrate the conclusions in chapter 4 and 5 with simulated data. 


\section{Acknowledgements}

The efforts of my master's thesis supervisor Dr. Mohamedou Ould Haye were most helpful in carrying on the thesis and its completion. Dr. Ould Haye not only helped me to set up the topic of my thesis, but also helped me to solve a lot of problems which led to a better understanding of books and papers.

During the past one and a half years in which I have been working on this thesis, my husband was always there to support me. Many thanks to him, Han Zhuo. 


\section{Table of Contents}

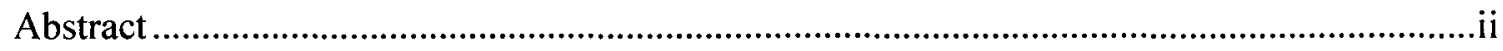

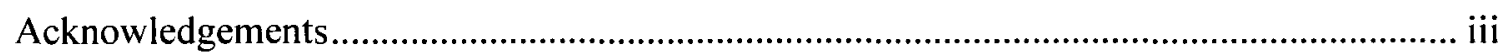

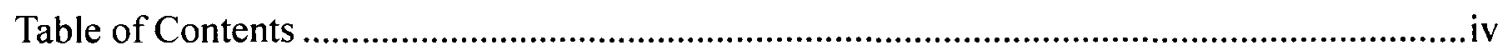

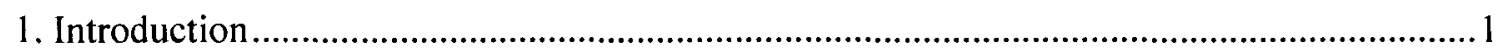

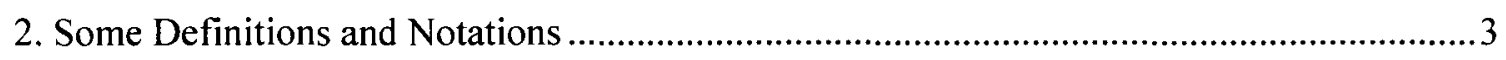

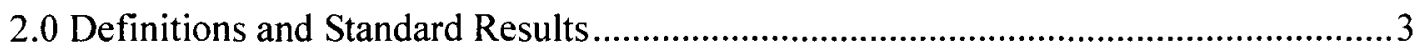

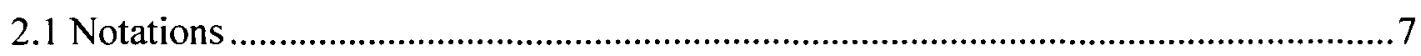

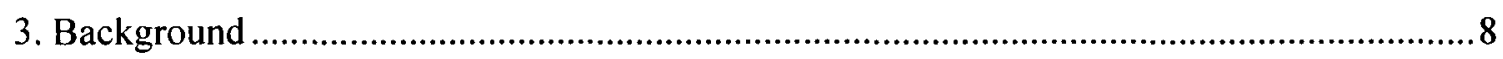

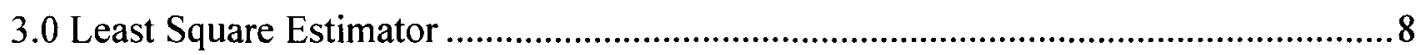

3.1 Best Linear Unbiased Estimator ......................................................................

4. Comparison of the Asymptotic Efficiency of the LSE with the BLUE in Linear Regression with Dependent Errors ........................................................................ 13

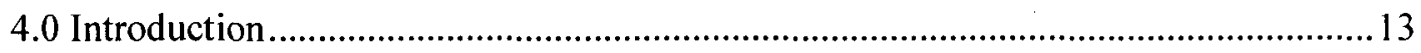

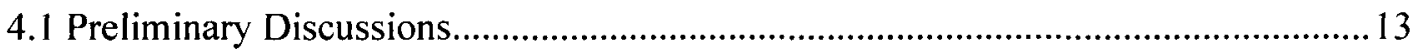

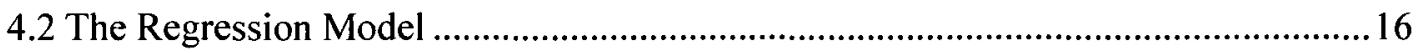

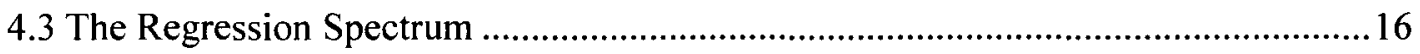

4.4 The Asymptotic Expressions for the Covariance Matrices .......................................22

4.5 Asymptotic Efficiency Conditions of LSE ........................................................... 31

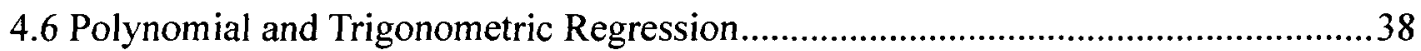

5. M-Estimation of Linear Regression with Dependent Errors ..........................................47

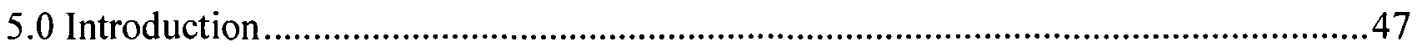

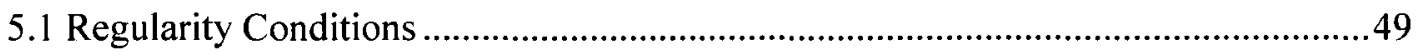

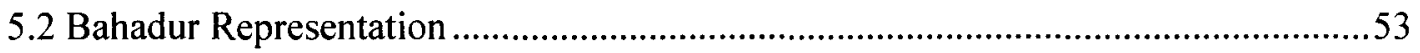

5.3 Application to Infinite Moving Average Errors ...................................................59

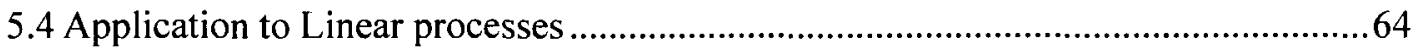

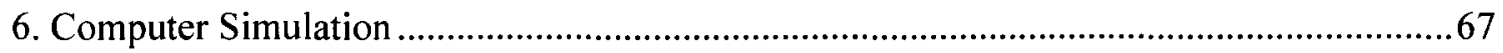

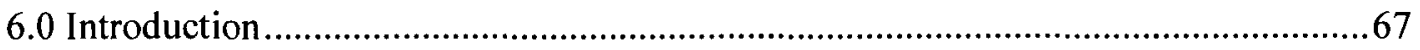

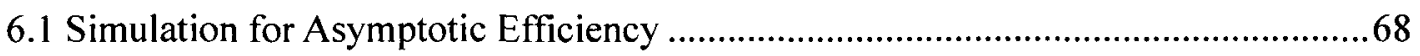

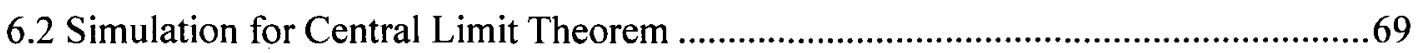

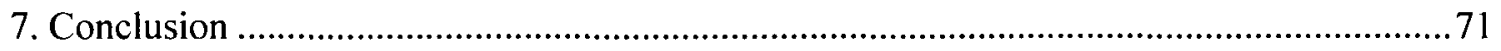

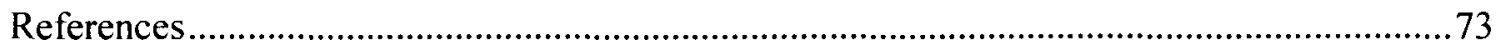

Appendix 1: Simulation Code and Result for Section 6.1 ..............................................75

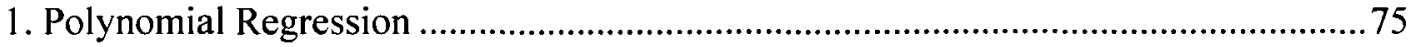

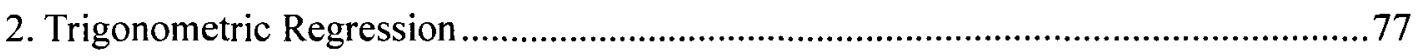

Appendix 2: Simulation Code and Result for Section 6.2 ........................................... 79 


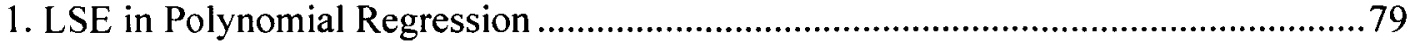

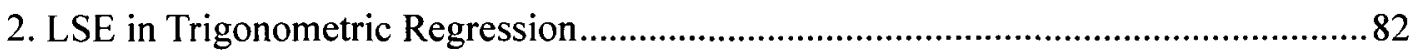

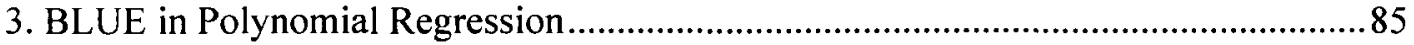

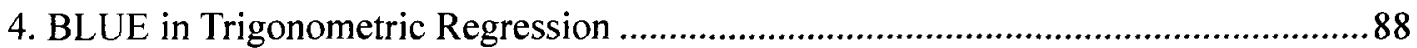




\section{Introduction}

A typical problem in regression analysis is estimating the regression coefficient $\beta$ when

$$
y_{1}=X_{1}^{\prime} \beta+\epsilon_{\imath},
$$

where $\epsilon_{t}$ is a stationary sequence and allowed to be an autocorrelated process. Usually, this problem is solved by applying the method of least squares (LS). It is well known that the least square estimator (LSE) is equal to the best linear unbiased estimator (BLUE) in the case of independent and identically distributed (i.i.d) errors.

The problem studied in this thesis arises when we are interested in a linear regression model with dependent errors. If the correlation matrix is known, then BLUE can be constructed. However, it is no longer true that the LSE coincides with BLUE for the models with dependent errors. In practice, we are interested in LSE, since the correlation matrix of the errors is seldom known. It is then natural to study whether LSE is asymptotically efficient with a large sample.

In this thesis, we will study if the LSE is asymptotically efficient under certain constraints. We assume that the errors are stationary and have absolutely continuous spectrum. Also, we study the asymptotic properties of M-estimators of regression parameters in linear models with dependent errors. LSE is a typical M-estimator. Then by applying LEMMA 2 in chapter 4, we can establish a central limit theorem (CLT) for both LSE and BLUE. Finally, to illustrate our conclusions, we generate a large set of data (in 
$\mathrm{R}$ language) and execute a computer simulation. The computer simulation results match the results from Chapter 3 and Chapter 4.

There are mainly three contributions that I made to this thesis:

- Chapter 3: Make details to Chapter 7 of Grenander and Rosenblatt (1984) by adding some minor proofs and calculations.

- Chapter 4: Derive CLT for LSE and BLUE.

- Chapter 5: All the computer simulation descriptions and codes. 


\section{Some Definitions and Notations}

\subsection{Definitions and Standard Results}

- Complex Conjugate: The conjugate of a complex number $z=a+i b$ where $a$ and $b$ are real numbers, is $\bar{z}=a-i b$.

- Nonnegative definite matrix: An $n \times n$ real symmetric matrix M is nonnegative definite if $z^{\prime} M z \geq 0$ for all vectors $z$ with real entries. Therefore, when we compare two matrices $\mathrm{A}$ and $\mathrm{B}$, we say $A \leq B$, if $B-A$ is nonnegative definite. The diagonal entries $m_{i j}$ of a nonnegative matrix are nonnegative and we have

$$
\left|m_{i j}\right| \leq \sqrt{m_{i i} m_{j j}} \leq \frac{m_{i i}+m_{j l}}{2},
$$

and thus

$$
\max \left|m_{i j}\right| \leq \max \left|m_{u}\right|
$$

- Nondecreasing Matrix: A matrix $T(\lambda)$ is said to be nondecreasing on an interval $I$ if $T\left(\lambda_{1}\right) \geq T\left(\lambda_{2}\right)$ for all $\lambda_{1}>\lambda_{2}$, where $\lambda_{1}, \lambda_{2} \in I$.

- Weakly Stationary Process: $\left\{z_{t}\right\}$ is (weakly) stationary if $E\left(z_{t}\right)$ is independent of $t$ and $\operatorname{Cov}\left(z_{t+h}, z_{1}\right)$ is independent of $t$ for each $h$ (Brockwell, P. J. \& Davis, R. A. (2002)).

- A function of bounded variation: The total variation of a real-valued function $f$ defined on an interval $[a, b] \in \mathbb{R}$ is the 
quantity $V_{b}^{a}(f)=\sup _{P \in \mathcal{P}} \sum_{i=0}^{n_{p}-1}\left|f\left(x_{i+1}\right)-f\left(x_{i}\right)\right|$, where $P=\left\{a=x_{0}<x_{1}<\cdots<x_{n_{p}}=b\right\}$ is a partition of $[a, b]$ and $\mathcal{P}$ is the class of all the partitions of $[a, b]$. If $f$ is differentiable and its derivative is integrable, its total variation is given by $V_{b}^{a}(f)=\int_{a}^{b}\left|f^{\prime}(x)\right| d x$. Hence $f$ is said to be of bounded variation on a chosen interval $[a, b]$ if its total variation is finite, i.e. $V_{b}^{a}(f)<\infty$. (Ambrosio, Fusco, \& Pallara (2000)).

- Hermitian matrix: Hermitian matrix is a square matrix $A$ such that $A=\bar{A}^{\prime}$.

- Herglotz's theorem: A complex-valued function $\gamma(\cdot)$ defined on the integers is nonnegative definite, if and only if

$$
\gamma(h)=\int_{-\pi, \pi]} e^{i h \lambda} d F(\lambda), \text { for all } h=0, \pm 1, \ldots,
$$

where $F(\cdot)$ is a right-continuous, nondecreasing, bounded function on $[-\pi, \pi]$ and $F(-\pi)=0$. (Brockwell, P. J. \& Davis, R. A. (1991)).

Since the covariance function $\gamma$ of any stationary process is nonnegative definite then it will have such a representation above. Here $\gamma(i-j)$ is nonnegative definite means for all vectors $a$ with real entries $a_{i}, i \in \mathbb{Z}$, we have $\sum_{i} \sum_{j} a_{i} \bar{a}_{j} \gamma(i-j) \geq 0$

- Projection Operator: A transformation $P$ on a vector space that satisfies $P=P^{2}$

- Generalized Parseval Equality: If $X$ is a separable Hilber space, $x, y \in X$, if 
$\left\{e_{n}\right\}$ is an orthonormal basis of $X$ and if $a_{n}=\left\langle x, e_{n}\right\rangle$ and $b_{n}=\left\langle y, e_{n}\right\rangle$ are the

Fourier coefficients of $x$ and $y$, then

$$
\langle x, y\rangle=\sum_{n=-\infty}^{\infty} a_{n} \bar{b}_{n}
$$

- Unitary Matrix (Orthogonal Matrix): A nonsingular complex matrix $Q$ is a unitary matrix if $Q^{\prime}=Q^{-1}$. In case of real matrix we say orthogonal instead.

- Schwarz Inequality in $\mathcal{L}^{2}$ :

$$
\left|\int g_{1}(x) g_{2}(x) d F(x)\right|^{2} \leq \int\left|g_{1}(x)\right|^{2} d F(x) \cdot \int\left|g_{2}(x)\right|^{2} d F(x)
$$

- Direct Sum of Matrices: The direct sum of $n$ square matrices $A_{1}, A_{2}, \ldots, A_{n}$ is the matrix $A$ given by

$$
\begin{aligned}
A & =A_{1} \oplus A_{2} \oplus \cdots \oplus A_{n} \\
& =\oplus_{i=1}^{n} A_{i} \\
& =\operatorname{diag}\left(A_{1}, A_{2}, \ldots, A_{n}\right) \\
& =\left[\begin{array}{llll}
A_{1} & & \\
& A_{2} & & \\
& & \ddots & \\
& & & A_{n}
\end{array}\right]
\end{aligned}
$$

We have 0 's on off diagonal blocks.

- Regression Equivariant: An estimator $T$ from a sample $\left(x_{i}, y_{1}\right), i=1, \ldots, n$, is called regression equivariant if

$$
T\left(\left\{\left(x_{i}, y_{i}+x_{i} v\right) ; i=1, \cdots, n\right\}\right)=T\left(\left\{\left(x_{i}, y_{i}\right) ; i=1, \cdots, n\right\}\right)+v
$$

where $v$ is any column vector (Rousseeuw and Leroy(2003), page 116).

- Convergence in probability: $X_{n} \stackrel{P}{\longrightarrow} X$ (i.e. $X_{n}$ converges to $X$ in 
probability) if $\forall \varepsilon>0, P\left[\left|X_{n}-X\right|>\varepsilon\right] \rightarrow 0$ as $n \rightarrow \infty$.

- Convergence in distribution: Let $X_{n}$ be a random variable with cumulative distribution function $F_{n}$. Let $X$ be a random variable with cumulative distribution function $F$. We say that $X_{n}$ converges in distribution to $X$ (and we write $\left.X_{n} \Rightarrow X\right)$ if $F_{n}(x) \rightarrow F(x)$ for all $x$ where $F$ is continuous.

- Remark on Convergence: Convergence in probability always implies convergence in distribution. However, if $X=a=$ constant, and $X_{n} \Rightarrow a$, then $X_{n} \stackrel{P}{\longrightarrow} a$

- Lebesgue dominated convergence theorem: Let $X_{n}$ and $X$ be random variables (R.V.), on a probability space $(\Omega, \mathcal{F}, P$ ). If there exists $Y \geq 0$ (a R.V.) such that $E[Y]<\infty,\left|X_{n}\right| \leq Y \quad$ and $\quad X_{n} \stackrel{P}{\longrightarrow} X$ then $E(|X|)<\infty$ and $E\left[X_{n}\right] \rightarrow E[X]$ (i.e. $\left.\int_{\Omega} X_{n} d P \rightarrow \int_{\Omega} X d P\right)$.

- Stochastically Lipschitz Process: A process $X(t)$ is stochastically Lispschitz if there exists $L \in \mathcal{L}^{1}$ (space of integrable random variables) such that $|X(t)-X(s)| \leq|t-s| L \quad \forall s, t$

- Jensen's Inequality: For any convex function $\varphi$ such that $\varphi(X)$ integrable and $\mathcal{A}=\sigma$-field, we have

$$
\varphi(E[X \mid \mathcal{A}]) \leq E[\varphi(X) \mid \mathcal{A}]
$$




\subsection{Notations}

- $p$-dimensional vector $v=\left(v_{1}, \cdots, v_{p}\right)^{\prime}:|v|=\left(\sum_{i=1}^{p} v_{i}^{2}\right)^{\frac{1}{2}}$;

- Random vector $V$ is said to be in $\mathcal{L}^{q}$, if $E\left(|v|^{q}\right)<\infty:\|V\|_{q}=\left[E\left(|v|^{q}\right)\right]^{\frac{1}{q}}$, $\|V\|=\|V\|_{2}, \quad \operatorname{var}(V)=E\left(V V^{\prime}\right)-E(V) E\left(V^{\prime}\right)$ which is the covariance matrix of $V$, the projection operators $\mathcal{P}_{k}, k \in \mathbb{Z}, \mathcal{P}_{k} V=E\left(V \mid \mathcal{F}_{k}\right)-E\left(V \mid \mathcal{F}_{k-1}\right), V \in \mathcal{L}^{1}$; where $\mathcal{F}_{k}$ is a nondecreasing sequence of $\sigma$-fields.

- For a sequence of random variables $\left(\eta_{n}\right)$ and a positive sequence $\left(d_{n}\right): \eta_{n}=o_{P}\left(d_{n}\right)$ if $\frac{\eta_{n}}{d_{n}} \stackrel{P}{\longrightarrow} 0$ and $\eta_{n}=O_{P}\left(d_{n}\right)$ if $\frac{\eta_{n}}{d_{n}}$ is bounded in probability (as $n \rightarrow \infty$ );

- A multivariate normal distribution with mean vector $\mu$ and covariance matrix $\sum$ is denoted as $N(\mu, \Sigma)$.

- Let $[X \mid \mathcal{F}]$ be the conditional distribution of $X$ given $\mathcal{F}$ : i.e. $\forall H \subset R$, $[X \mid \mathcal{F}](H)=P(X \in H \mid \mathcal{F})=E\left[1_{X \in H} \mid \mathcal{F}\right]$ 


\section{Background}

\subsection{Least Square Estimator}

An observed sample $y=\left\{y_{t}\right\}_{t=1}^{n}$ follows the regression model of the form

$$
y_{t}=X_{i}^{\prime} \beta+\epsilon_{1}
$$

for $t=1, \cdots, n$, where $X^{\prime}$ stands for transpose $X, X_{t}=\left(X_{t}^{(1)}, X_{t}^{(2)}, \ldots, X_{t}^{(p)}\right)^{\prime}$ is a $p \times 1$ vector of regression variables, and $\beta=\left(\beta_{1}, \beta_{2}, \ldots, \beta_{p}\right)^{\prime}$ is a $p \times 1$ vector of unknown regression coefficients, and $\epsilon=\left\{\epsilon_{t}\right\}_{t=1}^{n}$ is a vector of zero-mean stationary dependent errors with covariance matrix $R$.

We usually estimate the regression coefficients $\beta$ by applying the method of Least Squared errors. The least square estimator (LSE) $\hat{\beta}$ of $\beta$ is

$$
\hat{\beta}=\left(X^{\prime} X\right)^{-1} X^{\prime} y
$$

where $X=\left(X_{t}^{(j)}\right)_{t=1, \ldots, n ; j=1, \ldots, p}$.

The estimator $\hat{\beta}$ is unbiased since

$$
E[\hat{\beta}]=E\left[\left(X^{\prime} X\right)^{-1} X^{\prime} y\right]=\left(X^{\prime} X\right)^{-1} X^{\prime} X \beta=\beta
$$

Grenander and Rosenblatt (1984) solved $\hat{\beta}$ by minimizing the sum of squares $S(\beta)$ in the following method. Assuming that $X^{\prime} X$ is a nonsingular matrix, the sum of squared errors is given by 


$$
\begin{aligned}
S(\beta)= & (y-X \beta)^{\prime}(y-X \beta) \\
= & y^{\prime} y+\beta^{\prime} X^{\prime} X \beta-\beta^{\prime} X^{\prime} y-y^{\prime} X \beta \\
= & y^{\prime} y+\left(y^{\prime} X\left(X^{\prime} X\right)^{-1} X^{\prime} y-y^{\prime} X\left(X^{\prime} X\right)^{-1} X^{\prime} y\right)+\beta^{\prime}\left(X^{\prime} X\right)^{1 / 2}\left(X^{\prime} X\right)^{1 / 2} \beta \\
& -\beta^{\prime}\left(X^{\prime} X\right)^{1 / 2}\left(X^{\prime} X\right)^{-1 / 2} X^{\prime} y-y^{\prime} X\left(X^{\prime} X\right)^{1 / 2}\left(X^{\prime} X\right)^{-1 / 2} \beta \\
= & y^{\prime}\left(I-X\left(X^{\prime} X\right)^{-1} X^{\prime}\right) y-\beta^{\prime}\left(X^{\prime} X\right)^{1 / 2}\left(X^{\prime} X\right)^{-1 / 2} X^{\prime} y \\
& +\left(\left(X^{\prime} X\right)^{1 / 2} \beta-\left(X^{\prime} X\right)^{-1 / 2} X^{\prime} y\right)^{\prime}\left(\left(X^{\prime} X\right)^{1 / 2} \beta-\left(X^{\prime} X\right)^{-1 / 2} X^{\prime} y\right) \\
& =y^{\prime}\left(I-X\left(X^{\prime} X\right)^{-1} X^{\prime}\right) y+\left(\left(X^{\prime} X\right)^{1 / 2} \beta-\left(X^{\prime} X\right)^{-1 / 2} X^{\prime} y\right)^{\prime}\left(\left(X^{\prime} X\right)^{1 / 2} \beta-\left(X^{\prime} X\right)^{-1 / 2} X^{\prime} y\right) \\
\text { so } \hat{\beta}= & \left(X^{\prime} X\right)^{-1} X^{\prime} y \text { minimizes } S(\beta) .
\end{aligned}
$$

\subsection{Best Linear Unbiased Estimator}

Assume that $R^{-1}$ exists. The best linear unbiased estimator (BLUE) is another well known estimator of $\beta$. This unbiased estimator is a linear combination of the observed sample and has the minimum variance. The following idea of determining the expression of BLUE is from Montgomery, Peck and Vining (2001). As we know that $R$, the covariance matrix of $\epsilon$ is nonsingular, there exists an $n \times n$ nonsingular matrix $K$, such that $K K^{\prime}=R$. Setting $z=K^{-1} y, B=K^{-1} X$ and $g=K^{-1} \epsilon$, we have

$$
z_{1}=B_{\imath}^{\prime} \beta+g_{\imath}
$$

where for $t=1, \ldots, n, B_{t}=\left(B_{t}^{(1)}, B_{t}^{(2)}, \ldots, B_{t}^{(p)}\right)^{\prime}$ is a $p \times 1$ vector of regression variables, and $g=\left\{g_{t}\right\}_{t=1}^{n}$ is a vector of new errors that are still centered (i.e. with zero mean) but uncorrelated. We can prove this by

$$
E(g)=K^{-1} E(\epsilon)=0
$$


and

$$
\begin{aligned}
\operatorname{Cov}(g) & =E\left\{[g-E(g)][g-E(g)]^{\prime}\right\} \\
& =E\left(g g^{\prime}\right)-[E(g)][E(g)]^{\prime} \\
& =E\left(K^{-1} \epsilon \epsilon^{\prime} K^{\prime-1}\right)=K^{-1} E\left(\epsilon \epsilon^{\prime}\right) K^{\prime-1} \\
& =K^{-1} R\left(K^{\prime}\right)^{-1}=I .
\end{aligned}
$$

We can apply the same method of minimizing sum of squared errors as above to (3.1.1) to get the LSE of (3.1.1),

$$
\begin{aligned}
S(\beta)= & z^{\prime}\left(I-B\left(B^{\prime} B\right)^{-1} B^{\prime}\right) z \\
& +\left(\left(B^{\prime} B\right)^{\frac{1}{2}} \beta-\left(B^{\prime} B\right)^{-\frac{1}{2}} B^{\prime} z\right)^{\prime} \cdot\left(\left(B^{\prime} B\right)^{\frac{1}{2}} \beta-\left(B^{\prime} B\right)^{-\frac{1}{2}} B^{\prime} z\right),
\end{aligned}
$$

so $\left(B^{\prime} B\right)^{-1} B^{\prime} z=\left(X^{\prime} R^{-1} X\right)^{-1} X^{\prime} R^{-1} y$ minimizes $S(\beta)$.

We shall prove that this is the BLUE, i.e.

$$
\tilde{\beta}=\left(X^{\prime} R^{-1} X\right)^{-1} X^{\prime} R^{-1} y
$$

is a minimum variance linear unbiased estimator of $\beta$, where $\mathrm{R}$ is the $n \times n$ covariance matrix of $\epsilon$, assuming it to be nonsingular. First we prove that $\tilde{\beta}$ is unbiased,

$$
E \tilde{\beta}=\left(X^{\prime} R^{-1} X\right)^{-1} X^{\prime} R^{-1} X \beta=\beta
$$

We can also find the variance of $\tilde{\beta}$

$$
\begin{aligned}
\operatorname{Var}(\tilde{\beta}) & =\left(\left(X^{\prime} R^{-1} X\right)^{-1} X^{\prime} R^{-1}\right) R\left(\left(X^{\prime} R^{-1} X\right)^{-1} X^{\prime} R^{-1}\right)^{\prime} \\
& =\left(X^{\prime} R^{-1} X\right)^{-1}
\end{aligned}
$$

Let $\beta^{*}=L y$ be a linear combination of $y_{t}$ and an unbiased estimator of $\beta$, where $L=\left(l_{1}, l_{2}, \ldots, l_{n}\right)$. So we have for all $\beta$

$$
\beta=E\left(\beta^{*}\right)=E(L y)=E(L X \beta+L \epsilon)=E(L X \beta)=L X \beta
$$

and thus 


$$
L X=I
$$

We can also find the covariance matrix of $\beta^{*}$, which is

$$
\operatorname{Var}\left(\beta^{*}\right)=L R L^{\prime}
$$

We refer to Grenander and Rosenblatt's (1984) idea, where the proof is given in the following approach. The proof requires the following Schwarz inequality for matrices:

Let $Q, U$ be two $n \times s$ matrices such that $Q^{\prime} Q$ is nonsingular. Then we have

$$
U^{\prime} U \geq\left(Q^{\prime} U\right)^{\prime}\left(Q^{\prime} Q\right)^{-1}\left(Q^{\prime} U\right)
$$

There is equality if and only if there is a $s \times s$ matrix $V$ such that $U=Q V$. The proof of (3.1.3) is given in the following: Let $\lambda, \mu$ be any $s \times 1$ vectors, then

$$
\begin{aligned}
& \left(\lambda^{\prime} Q^{\prime}+\mu^{\prime} U^{\prime}\right)\left(\lambda^{\prime} Q^{\prime}+\mu^{\prime} U^{\prime}\right)^{\prime} \geq 0 \\
& \Rightarrow \lambda^{\prime} Q^{\prime} Q \lambda+\lambda^{\prime} Q^{\prime} U \mu+\mu^{\prime} U^{\prime} Q \lambda+\mu^{\prime} U^{\prime} U \mu \geq 0 .
\end{aligned}
$$

Let $A=Q^{\prime} Q, B=Q^{\prime} U, C=U^{\prime} U$.

$$
\begin{aligned}
& \Rightarrow \lambda^{\prime} A \lambda+\lambda^{\prime} B \mu+\mu^{\prime} B^{\prime} \lambda+\mu^{\prime} C \mu \geq 0 \\
& \Rightarrow\left(\lambda^{\prime} A^{\frac{1}{2}}+\mu^{\prime} B^{\prime} A^{-\frac{1}{2}}\right)\left(\lambda^{\prime} A^{\frac{1}{2}}+\mu^{\prime} B^{\prime} A^{-\frac{1}{2}}\right)^{\prime}+\mu^{\prime}\left[C-B^{\prime} A^{-1} B\right] \mu \geq 0 .
\end{aligned}
$$

Setting $\lambda^{\prime}=-\mu^{\prime} B^{\prime} A^{-1}$, we have

$$
\lambda^{\prime} A^{\frac{1}{2}}+\mu^{\prime} B^{\prime} A^{-\frac{1}{2}}=-\mu^{\prime} B^{\prime} A^{-1} A^{\frac{1}{2}}+\mu^{\prime} B^{\prime} A^{-\frac{1}{2}}=0 .
$$

Thus,

$$
C \geq B^{\prime} A^{-1} B
$$

which is equivalent to (3.1.3).

Recall the variance of $\beta^{*}$, let $U=R^{\frac{1}{2}} L^{\prime}, Q^{\prime}=X^{\prime} R^{-\frac{1}{2}}$. Apply the inequality (3.1.3) and the fact that $L X=I$, so we have 


$$
\begin{aligned}
\operatorname{Var}\left(\beta^{*}\right) & =E\left(\beta^{*}-\beta\right)\left(\beta^{*}-\beta\right)^{\prime} \\
& =L R L^{\prime}=\left(L R^{1 / 2}\right)\left(L R^{1 / 2}\right)^{\prime} \\
& \geq(L X)\left(X^{\prime} R^{-1} X\right)^{-1}(L X)^{\prime} \\
& =\left(X^{\prime} R^{-1} X\right)^{-1} \\
& =E(\tilde{\beta}-\beta)(\tilde{\beta}-\beta)^{\prime} .
\end{aligned}
$$

Therefore, $\tilde{\beta}$ has minimum variance. 


\section{Comparison of the Asymptotic Efficiency of the LSE with the BLUE in Linear Regression with Dependent Errors}

\subsection{Introduction}

In this section, we wish to demonstrate the asymptotic efficiency of the LSE relative to the BLUE, i.e. asymptotically the LSE will have same covariance matrix as the BLUE. The main idea follows Chapter 7 of Grenander and Rosenblatt (1984). We shall deal with stationary errors with piecewise continuous and positive spectral density.

\subsection{Preliminary Discussions}

For a simple linear regression model,

$$
y_{t}=\beta x_{t}+\epsilon_{t}, \quad t=1, \cdots, n
$$

we wish to estimate the regression coefficient $\beta, y_{t}, x_{t}$, and $\epsilon_{t}$ are complex valued. Here errors are allowed to be dependent. We say that $\beta_{n}$ is a sequence of linear consistent estimators of $\beta$,

$$
\beta_{n}=\sum_{i=1}^{n} \alpha_{t}^{(n)} y_{l}
$$

if $E\left|\beta_{n}-\beta\right|^{2} \rightarrow 0$ as $n \rightarrow \infty$, i.e. $\beta_{n}$ converges to $\beta$ in $L^{2}$ (in the squared mean). 
We assume that the spectral density $f$ of errors exists and is piecewise continuous and positive. The covariance of errors is given by

$$
\begin{aligned}
E\left(\epsilon_{s} \epsilon_{t}\right) & =r_{s-t} \\
& =\int_{\pi}^{\pi} e^{i(s-t) \lambda} f(\lambda) d \lambda .
\end{aligned}
$$

We can write the mean squared error in the form

$$
\begin{aligned}
E\left|\beta_{n}-\beta\right|^{2} & =E\left|\beta_{n}-E \beta_{n}+E \beta_{n}-\beta\right|^{2} \\
& =E\left|\beta_{n}-E \beta_{n}\right|^{2}+\left|E \beta_{n}-\beta\right|^{2}+2 E\left[\left(\beta_{n}-E \beta_{n}\right)\left(E \beta_{n}-\beta\right)\right] \\
& =E\left|\beta_{n}-E \beta_{n}\right|^{2}+\left|E \beta_{n}-\beta\right|^{2} \\
& =E\left|\beta_{n}-\sum_{t=1}^{n} \alpha_{t}^{(n)} x_{t} \beta\right|^{2}+\left|\sum_{t=1}^{n} \alpha_{t}^{(n)} x_{t} \beta-\beta\right|^{2} \\
& =E\left|\sum_{t=1}^{n} \alpha_{t}^{(n)} \epsilon_{t}\right|^{2}+|\beta|^{2}\left|\sum_{t=1}^{n} \alpha_{t}^{(n)} x_{t}-1\right|^{2} \\
& =I_{\pi}^{\pi}\left|\sum_{t=1}^{n} \alpha_{t}^{(n)} e^{i t \lambda}\right|^{2} f(\lambda) d \lambda+|\beta|^{2}\left|\sum_{t=1}^{n} \alpha_{t}^{(n)} x_{t}-1\right|^{2} .
\end{aligned}
$$

Thus in order to have a consistent estimate, we must have

$$
\lim _{n \rightarrow \infty} \sum_{i=1}^{n} \alpha_{t}^{n} x_{t}=1
$$

As

$$
\begin{aligned}
& \lim _{n \rightarrow \infty} \int_{-\pi}^{\pi}\left|\sum_{t=1}^{n} \alpha_{t}^{(n)} e^{i t \lambda}\right|^{2} f(\lambda) d \lambda \\
& \geq \lim _{n \rightarrow \infty} \int_{-\pi}^{\pi}\left|\sum_{t=1}^{n} \alpha_{t}^{(n)} e^{i t \lambda}\right|^{2} \min _{\lambda} f(\lambda) d \lambda=\lim _{n \rightarrow \infty} \min _{\lambda} f(\lambda) \int_{-\pi}^{\pi} \sum_{s, t=1}^{n} \alpha_{t}^{(n)} \bar{\alpha}_{s}^{(n)} e^{i(t-s) \lambda} d \lambda \\
& =\lim _{n \rightarrow \infty} \min f(\lambda) \sum_{\lambda}^{n} \alpha_{t}^{(n)} \bar{\alpha}_{s}^{(n)} \int_{-\pi}^{\pi} e^{i(t-s) \lambda} d \lambda=\lim _{n \rightarrow \infty} 2 \pi \min _{\lambda} f(\lambda) \sum_{t=1}^{n}\left|\alpha_{t}^{(n)}\right|^{2},
\end{aligned}
$$

since $\int_{\pi}^{\pi} e^{i(t-s) \hat{\lambda}} d \lambda=0$, if $t \neq s$. Therefore, assuming consistency of $\beta_{n}$

$$
\lim _{n \rightarrow \infty} \sum_{t=1}^{n}\left|\alpha_{t}^{(n)}\right|^{2}=0
$$


By Schwarz inequality,

$$
1=1^{2}=\left(\lim _{n \rightarrow \infty} \sum_{t=1}^{n} \alpha_{t}^{(n)} x_{t}\right)^{2} \leq \lim _{n \rightarrow \infty} \sum_{t=1}^{n}\left|\alpha_{t}^{(n)}\right|^{2} \cdot \sum_{t=1}^{n}\left|x_{t}\right|^{2}
$$

which implies therefore that

$$
\lim _{n \rightarrow \infty} \sum_{t=1}^{n}\left|x_{t}\right|^{2}=\infty
$$

is a necessary condition for the estimator $\hat{\beta}$ to be consistent. This condition is also sufficient in the presence of (4.1.3) for the LSE by the following argument. Here if we take

$$
\beta_{n}=\frac{\sum_{t=1}^{n} \bar{x}_{t} y_{t}}{\sum_{t=1}^{n}\left|\bar{x}_{t}\right|^{2}}
$$

so the asymptotic mean square is

$$
\begin{aligned}
& \lim _{n \rightarrow \infty} \int_{-\pi}^{\pi}\left|\sum_{i=1}^{n} \alpha_{i}^{(n)} e^{i t \lambda}\right|^{2} f(\lambda) d \lambda \\
& \leq \lim _{n \rightarrow \infty} 2 \pi \max _{\lambda} f(\lambda) \sum_{t=1}^{n}\left|\alpha_{t}^{(n)}\right|^{2} \\
& =\lim _{n \rightarrow \infty} 2 \pi \max _{\lambda} f(\lambda) \sum_{t=1}^{n}\left|\frac{\bar{x}_{t}}{\sum_{t=1}^{n}\left|x_{t}\right|^{2}}\right|^{2} \\
& =\lim _{n \rightarrow \infty} 2 \pi \max _{\lambda} f(\lambda) \frac{\sum_{t=1}^{n}\left|\bar{x}_{t}\right|^{2}}{\left(\sum_{i=1}^{n}\left|x_{t}\right|^{2}\right)^{2}} \\
& =\lim _{n \rightarrow \infty} 2 \pi \max _{\lambda} f(\lambda) \frac{1}{\sum_{i=1}^{n}\left|x_{t}\right|^{2}}=0 .
\end{aligned}
$$

This condition will be applied in the following discussion. 


\subsection{The Regression Model}

We shall work on a multiple regression model $y=X \beta+\epsilon$ for the rest of this section. Here, the n-observation vector is $y=\left(y_{1}, y_{2}, \cdots, y_{n}\right)^{\prime}$, the regression vectors $\operatorname{are} X^{(f)}=\left(X_{1}^{(j)}, X_{2}^{(j)}, \cdots, X_{n}^{(j)}\right)^{\prime}, j=1,2, \cdots p$, and $X=\left(X^{(1)}, X^{(2)}, \cdots, X^{(p)}\right)$.

We investigate the conditions under which the least square estimators of the regression coefficients are asymptotically efficient relative to BLUE, if the regression is a polynomial or trigonometric regression.

\subsection{The Regression Spectrum}

For a fixed $v, v=1,2, \cdots, p$, let

$$
\Phi_{n}^{(v)}=\sum_{t=1}^{n}\left|X_{t}^{(v)}\right|^{2}=\left|X^{(v)}\right|^{2}
$$

where $\Phi_{n}^{(1)}$ approaches infinity as $n \rightarrow \infty$, otherwise as we discussed in section 4.1 LSE is not a consistent estimate of $\beta$. In addition, we assume that $\Phi_{n}^{(v)}$ is slowly varying in $n$ as $n \rightarrow \infty$, i.e. for $v=1, \ldots, p$

$$
\lim _{n \rightarrow \infty} \frac{\Phi_{n+h}^{(1)}}{\Phi_{n}^{(v)}}=1
$$

for every h. For example, by letting $\Phi_{n}^{(v)}=\log n$, we have the slowly varying property 
for $\lim _{n \rightarrow \infty} \frac{\log n+h}{\log n}=1$. We will also assume that

$$
R_{h}^{(r, s)}=\lim _{n \rightarrow \infty} \frac{\sum_{t=1}^{n} X_{t+h}^{(r)} \bar{X}_{t}^{(s)}}{\sqrt{\Phi_{n}^{(r)} \Phi_{n}^{(s)}}}
$$

exists for $1 \leq r, s \leq p$ and all integers $h$ and that $X_{t}^{(r)}=0$ for $t \leq 0$. Then, we have

$$
\begin{aligned}
R_{-h}^{(r, s)} & =\lim _{n \rightarrow \infty} \frac{\sum_{t=1}^{n} X_{t-h}^{(r)} \bar{X}_{t}^{(s)}}{\sqrt{\Phi_{n}^{(r)} \Phi_{n}^{(s)}}} \\
& =\lim _{n \rightarrow \infty} \frac{\sum_{u=1}^{n-h} X_{u}^{(r)} \bar{X}_{u+h}^{(s)}}{\sqrt{\Phi_{n-h}^{(r)} \Phi_{n-h}^{(s)}}} \sqrt{\frac{\Phi_{n-h}^{(r)} \Phi_{n-h}^{(s)}}{\Phi_{n}^{(r)} \Phi_{n}^{(s)}}} \\
= & \lim _{n \rightarrow \infty} \frac{\sum_{u=1}^{n-h} \bar{X}_{u+h}^{(s)} X_{u}^{(r)}}{\sqrt{\Phi_{n-h}^{(r)} \Phi_{n-h}^{(s)}}} .
\end{aligned}
$$

By applying the slowly increasing property of $\Phi_{n}^{(v)}$ (see (4.3.2)), the last limit can be written as

$$
\lim _{n \rightarrow \infty} \frac{\sum_{n=1}^{n-h} \bar{X}_{u+h}^{(s)} X_{u}^{(r)}}{\sqrt{\Phi_{n-h}^{(r)} \Phi_{n-h}^{(s)}}}=\lim _{n \rightarrow \infty} \frac{\sum_{u=1}^{n} \bar{X}_{u+h}^{(s)} X_{u}^{(r)}}{\sqrt{\Phi_{n}^{(r)} \Phi_{n}^{(s)}}}=\bar{R}_{h}^{(s, r)},
$$

and hence we get

$$
R_{-h}^{(r, s)}=\bar{R}_{h}^{(s, r)} \text {, i.e., } R_{-h}=\bar{R}_{h}^{\prime}
$$

where $R_{-h}$ and $\vec{R}_{h}^{\prime}$ are matrices. Let us consider for any $p \times 1$ vector $\alpha$, the quadratic form: 


$$
\begin{aligned}
\sigma_{v-\mu} & =\alpha^{\prime} R_{v-\mu} \alpha \\
& =\lim _{n \rightarrow \infty} \sum_{r, s=1}^{p} \bar{\alpha}_{r} \sum_{t=1}^{n} \frac{X_{t+v}^{(r)} \bar{X}_{t+\mu}^{(s)}}{\sqrt{\Phi_{n}^{(r)} \Phi_{n}^{(s)}}} \alpha_{s} \\
& =\lim _{n \rightarrow \infty} \sum_{r, s=1}^{p} \frac{\bar{\alpha}_{r}}{\sqrt{\Phi_{n}^{(r)}}} \sum_{t=1}^{n} X_{t+v}^{(r)} \bar{X}_{t+\mu}^{(s)} \frac{\alpha_{s}}{\sqrt{\Phi_{n}^{(s)}}} .
\end{aligned}
$$

Let $\gamma$ be $k \times 1$ vector and $k$ is arbitrary. Since the sequence (in $\mathrm{n}$ ) $\alpha^{\prime} R_{u-\mu} \alpha$ is nonnegative-definite,

$$
\begin{aligned}
\sum_{\nu, \mu=1}^{k} \bar{\gamma}_{\nu} \sigma_{v-\mu} \gamma_{\mu} & =\lim _{n \rightarrow \infty} \sum_{\nu, \mu=1}^{k} \bar{\gamma}_{\nu} \sum_{r, s=1}^{p} \frac{\bar{\alpha}_{r}}{\sqrt{\Phi_{n}^{(r)}}} \sum_{t=1}^{n} X_{t+\nu}^{(r)} \bar{X}_{t+\mu}^{(s)} \frac{\alpha_{s}}{\sqrt{\Phi_{n}^{(s)}}} \gamma_{\mu} \\
& =\lim _{n \rightarrow \infty} \sum_{t=1}^{n} \sum_{v, \mu=1}^{k} \sum_{r, s=1}^{p} \bar{\gamma}_{v} \frac{\bar{\alpha}_{r}}{\sqrt{\Phi_{n}^{(r)}}} X_{t+\nu}^{(r)} \bar{X}_{t+\mu}^{(s)} \frac{\alpha_{s}}{\sqrt{\Phi_{n}^{(s)}}} \gamma_{\mu} \\
& =\lim _{n \rightarrow \infty} \sum_{t=1}^{n}\left|\sum_{v, r} X_{t+v}^{(r)} \frac{\bar{\alpha}_{r} \bar{\gamma}_{v}}{\sqrt{\Phi_{n}^{(r)}}}\right|^{2} \geq 0,
\end{aligned}
$$

the spectral representation of $R_{h}$ is given below

$$
R_{h}=\int_{-\pi}^{\pi} e^{i h \lambda} d M(\lambda)
$$

where $\Delta M(\lambda)=M\left(\lambda_{2}\right)-M\left(\lambda_{1}\right)$ is a nonnegative-definite matrix for every interval $\left(\lambda_{1}, \lambda_{2}\right)$. We will prove this below.

Assume that $T=\{1,2, \ldots, n\}$ and $z_{t}=\left(z_{t}^{(1)}, z_{t}^{(2)}, \ldots, z_{t}^{(m)}\right)$ is a weakly stationary process taking values in an m-dimension space with zero mean. We introduce its covariance matrix

$$
\begin{aligned}
R(t) & =\left\{r_{j, k}(t)\right\} \\
& =\left\{E\left(z_{s+t}^{(j)} \bar{z}_{s}^{(k)}\right) ; j, k=1,2, \ldots, m\right\}, \quad s, t \in T
\end{aligned}
$$

where $R(-t)=\bar{R}^{\prime}(t)$. Let $\alpha=\left(\alpha_{1}, \alpha_{2}, \ldots, \alpha_{m}\right)^{\prime}$ be an arbitrary $m \times 1$ vector. Then $\alpha^{\prime} \cdot z_{t}$ is complex-valued and weakly stationary process. Then by applying the Herglotz's 
theorem we have

$$
E\left(\alpha^{\prime} z_{s}\right) \overline{\left(\alpha^{\prime} z_{t}\right)}=\int_{-\pi}^{\pi} e^{i(s-t) \lambda} d M_{\alpha}(\lambda)
$$

where the spectral distribution function $M_{\alpha}(\lambda)$ depends on $\alpha$. Also, we have

$$
\begin{aligned}
E\left(\alpha^{\prime} z_{s}\right) \overline{\left(\alpha^{\prime} z_{t}\right)} & =\sum_{\nu, \mu=1}^{m} \alpha_{\nu} \bar{\alpha}_{\mu} r_{\nu, \mu}(s-t) \\
& =\alpha^{\prime} R(s-t) \alpha .
\end{aligned}
$$

Then $M_{\alpha}(\lambda)=\alpha^{\prime} M(\lambda) \alpha$, where $M(\lambda)$ is a $m \times m$ Hermitian matrix. The increments over intervals are

$$
\alpha^{\prime} \Delta M(\lambda) \alpha=\Delta M_{\alpha}(\lambda) \geq 0
$$

since $M_{\alpha}(\lambda)$ is non-decreasing. So $\Delta M(\lambda)$ is a nonnegative definite matrix. Taking the equations in (4.3.5) and (4.3.6) to be equal for all $\alpha$, we get

$$
\begin{aligned}
& \sum_{j, k=1}^{m} \alpha_{j} \bar{\alpha}_{k} r_{j, k}(s-t)=\int_{-\pi}^{\pi} e^{i(s-t) \lambda} d M_{\alpha}(\lambda) \\
& \Rightarrow \sum_{j, k=1}^{m} \alpha_{j} \bar{\alpha}_{k} r_{j, k}(s-t)=\sum_{j, k=1}^{m} \alpha_{j} \bar{\alpha}_{k} \int_{-\pi}^{\pi} e^{i(s-t) \lambda} d M_{j, k}(\lambda) \\
& \Rightarrow r_{j, k}(s-t)=\int_{-\pi}^{\pi} e^{i(s-t) \lambda} d M_{j, k}(\lambda)
\end{aligned}
$$

where $M_{j, k}(\lambda)$ is the element in the matrix $M(\lambda)$ corresponding to entry $(j, k)$.

Taking $\alpha_{j}=1, \alpha_{\nu}=0$ for $v \neq j$, we get from (4.3.7)

$$
\Delta M_{j j}(\lambda) \geq 0
$$

so that the diagonal elements of $M(\lambda)$ are non-decreasing functions. As $M_{j j}(\pi)-M_{j j}(-\pi)=r_{j j}(0)<\infty$ (by assumption (4.3.3) that $R_{h}$ exists), we see that the diagonal elements are also bounded. 
Again, taking $\alpha_{j}=a, \alpha_{k}=b, \alpha_{v}=0$ for $v \neq j, k$, we get from (4.3.7)

$$
\begin{aligned}
& a^{2} \Delta M_{j j}(\lambda)+2 a b \Delta M_{j k}(\lambda)+b^{2} \Delta M_{k k}(\lambda) \geq 0 \\
& \Rightarrow\left(a \sqrt{\Delta M_{j j}(\lambda)}+b \sqrt{\Delta M_{k k}(\lambda)}\right)^{2}+2 a b \Delta M_{j k}(\lambda)-2 a b \sqrt{\Delta M_{j j}(\lambda) \Delta M_{k k}(\lambda)} \geq 0
\end{aligned}
$$

For any $a$ and $b$, in setting $\left(a \sqrt{\Delta M_{j j}(\lambda)}+b \sqrt{\Delta M_{k k}(\lambda)}\right)^{2}=0, a$ and $b$ must have different signs. Hence, we have

$$
\begin{aligned}
& 2 a b \Delta M_{j k}(\lambda) \geq 2 a b \sqrt{\Delta M_{j j}(\lambda) \Delta M_{k k}(\lambda)} \\
& \Rightarrow\left|\Delta M_{j k}(\lambda)\right| \leq \sqrt{\Delta M_{j j}(\lambda) \Delta M_{k k}(\lambda)} .
\end{aligned}
$$

Since $M_{j j}(\lambda)$ and $M_{k k}(\lambda)$ are bounded non-decreasing functions, $\Delta M_{j k}(\lambda)$ is bounded, and hence all the elements of $M(\lambda)$ are of bounded variation. For $j \neq k, M_{j k}(\lambda)$ is not necessarily non-decreasing. Therefore, we have proved the spectral representation

$$
R_{s-t}=\int_{-\pi}^{\pi} e^{i(s-t) \lambda} d M(\lambda)
$$

where $M_{j j}(\lambda)$ is the spectral distribution function of the $j$ th component of $z_{i}$ and $M_{j k}(\lambda)$ is called the cross spectral distribution function of the $j$ th and $k$ th components. Reciprocally, similarly to 1-dimention Herglotz's theorem, any matrix $R_{s-1}$ having (4.3.8) representation can be considered as the covariance function of a stationary vector process.

Therefore, according to the spectral representation in (4.3.4), $M(\lambda)$ is the spectral distribution function of the limiting matrix $R_{h}$ generated from the regression vectors $X_{l}$, and we assume that

$$
R_{0}=M(\pi)-M(-\pi)=M
$$

is nonsingular. 
There should be no confusion between the $p \times p$ limit matrix $R_{h}$ built from the design $X_{t}$, and the $n \times n$ covariance matrix $\mathrm{R}$ of errors $\epsilon_{t}$.

As we proved in the previous section,

$$
\hat{\beta}=\left(X^{\prime} X\right)^{-1} X^{\prime} y,
$$

and

$$
\tilde{\beta}=\left(X^{\prime} R^{-1} X\right)^{-1} X^{\prime} R^{-1} y
$$

are LSE and BLUE, respectively. Then,

$$
\begin{aligned}
& E(\hat{\beta}-\beta)(\hat{\beta}-\beta)^{\prime}=\left(X^{\prime} X\right)^{-1} X^{\prime} R X\left(X^{\prime} X\right)^{-1}, \\
& E(\tilde{\beta}-\beta)(\tilde{\beta}-\beta)^{\prime}=\left(X^{\prime} R^{-1} X\right)^{-1} .
\end{aligned}
$$

We know that

$$
E(\hat{\beta}-\beta)(\hat{\beta}-\beta)^{\prime} \geq E(\tilde{\beta}-\beta)(\tilde{\beta}-\beta)^{\prime}
$$

Hence, we say that $\hat{\beta}$ is asymptotically efficient if

$$
\lim _{n \rightarrow \infty} D_{n} E(\hat{\beta}-\beta)(\hat{\beta}-\beta)^{\prime} D_{n}=\lim _{n \rightarrow \infty} D_{n} E(\tilde{\beta}-\beta)(\tilde{\beta}-\beta)^{\prime} D_{n},
$$

where $D_{n}$ is a diagonal matrix

$$
D_{n}=\operatorname{diag}\left(\sqrt{\Phi_{n}^{(1)}}, \sqrt{\Phi_{n}^{(2)}}, \ldots, \sqrt{\Phi_{n}^{(p)}}\right)
$$

To assure that the limiting matrices remain finite and positive (do not vanish) as $n \rightarrow \infty$, we right and left multiplied the covariance matrices by $D_{n}$. Let $\lambda$ be the points for which there exist $\lambda_{1}, \lambda_{2}: \lambda_{1}<\lambda<\lambda_{2}$ and $M\left(\lambda_{2}\right)-M\left(\lambda_{1}\right)=\Delta M(I)>0 \quad$ (where $\left.I=\left(\lambda_{1}, \lambda_{2}\right)\right)$. Such points $\lambda$ are called the regression spectrum and are denoted by $S$. 


\subsection{The Asymptotic Expressions for the Covariance} Matrices

We are interested in comparing the least square estimators $\hat{\beta}$ and best linear unbiased estimators $\tilde{\beta}$ of $\beta$ as $n \rightarrow \infty$. In theory, $\tilde{\beta}$ has the minimum variance among all unbiased linear estimators, but it is not very practical. First, usually $R$ is unknown, although it can be estimated if we have sufficiently large sample. Secondly, $R$ is practically quite difficult to invert. However, in some important types of regression, the least square estimator $\hat{\beta}=\left(X^{\prime} X\right)^{-1} X^{\prime} y$ is found asymptotically efficient for all positive and piecewise continuous spectral densities $f(\lambda)$. The $p \times p$ matrix $X^{\prime} X$ is much easier to invert than the $n \times n$ matrix $R$, and we know that from (3.0.3) $\hat{\beta}$ is unbiased.

\section{The Asymptotic Expressions for the Covariance Matrices of LSE}

We first consider the asymptotic behavior of the matrix involved in the asymptotic LSE,

$$
D_{n} E(\hat{\beta}-\beta)(\hat{\beta}-\beta)^{\prime} D_{n}=D_{n}\left(X^{\prime} X\right)^{-1} X^{\prime} R X\left(X^{\prime} X\right)^{-1} D_{n}
$$

Let us consider a moving average example in this part. Assume that the errors, have an $M A(q)$, moving average form,

$$
\epsilon_{t}=\sum_{j=0}^{q} a_{j} Z_{t-j}=\theta(B) Z_{t}
$$

where $\left\{Z_{1}\right\}$ is white noise with variance one and $\theta(B)=\sum_{j=0}^{q} a_{j} B^{\prime}$, where $B$ is the shift 
operator $\left(B^{\prime} Z_{t}=Z_{t-j}\right)$. Then the spectral density of the errors is

$$
f(\lambda)=\frac{\sigma^{2}}{2 \pi}\left|\theta\left(e^{-i \lambda}\right)\right|^{2}=\sum_{h=-q}^{q} f_{h} e^{i h \lambda},
$$

which is a trigonometric polynomial. Now, we know that

$$
f(\lambda)=\frac{1}{2 \pi} \sum_{h=-\infty}^{\infty} e^{i h \lambda} r_{h}=\frac{1}{2 \pi} \sum_{h=-q}^{q} e^{i h \lambda} r_{h},
$$

so the covariance is

$$
r_{h}=\left\{\begin{array}{l}
2 \pi f_{h},|h| \leq q \\
0,|h|>q .
\end{array}\right.
$$

We can write (4.4.1) in the following way,

$$
\begin{aligned}
& D_{n}\left(X^{\prime} X\right)^{-1} X^{\prime} R X\left(X^{\prime} X\right)^{-1} D_{n} \\
& =\left(D_{n}\left(X^{\prime} X\right)^{-1} D_{n}\right)\left(D_{n}^{-1} X^{\prime} R X D_{n}^{-1}\right)\left(D_{n}\left(X^{\prime} X\right)^{-1} D_{n}\right) .
\end{aligned}
$$

Then we can get

$$
\begin{aligned}
\left\{D_{n}^{-1} X^{\prime} R X D_{n}^{-1}\right\}_{\nu \mu} & =\frac{\sum_{j, k=1}^{n} \bar{X}_{j}^{(\cdot)} r_{j-k} X_{k}^{(\mu)}}{\sqrt{\Phi_{n}^{(\nu)} \Phi_{n}^{(\mu)}}}, \text { let } j-k=h \in(-q, q) \\
& =2 \pi\left(\sum_{h=0}^{q} f_{h} \frac{\sum_{k=1}^{n-h} X_{k}^{(\mu)} \bar{X}_{h+k}^{(\nu)}}{\sqrt{\Phi_{n}^{(\nu)} \Phi_{n}^{(\mu)}}}+\sum_{h=-q}^{-1} f_{h} \frac{\sum_{j=1}^{n+h} X_{j-h}^{(\mu)} \bar{X}_{j}^{(\nu)}}{\sqrt{\Phi_{n}^{(\nu)} \Phi_{n}^{(\mu)}}}\right)
\end{aligned}
$$

Taking the limit of the above equation as $n \rightarrow \infty$ and using the slowly increasing property of $\Phi_{n}^{(v)}$, we get 


$$
\begin{aligned}
\lim _{n \rightarrow \infty}\left\{D_{n}^{-1} X^{\prime} R X D_{n}^{-1}\right\}_{\nu \mu} & =\lim _{n \rightarrow \infty} 2 \pi\left(\sum_{h=0}^{q} f_{h} \frac{\sum_{k=1}^{n-h} X_{k}^{(\mu)} \bar{X}_{h+k}^{(\nu)}}{\sqrt{\Phi_{n}^{(\nu)} \Phi_{n}^{(\mu)}}}+\sum_{h=-q}^{-1} f_{h} \frac{\sum_{j=1}^{n+h} X_{j-h}^{(\mu)} \bar{X}_{j}^{(\nu)}}{\sqrt{\Phi_{n}^{(\nu)} \Phi_{n}^{(\mu)}}}\right) \\
& =2 \pi\left(\sum_{h=0}^{q} f_{h} \lim _{n \rightarrow \infty} \frac{\sum_{k=1}^{n-h} X_{k}^{(\mu)} \bar{X}_{h+k}^{(\nu)}}{\sqrt{\Phi_{n}^{(\nu)} \Phi_{n}^{(\mu)}}}+\sum_{h=-q}^{-1} f_{h} \lim _{n \rightarrow \infty} \frac{\sum_{j=1}^{n+h} X_{j-h}^{(\mu)} \bar{X}_{j}^{(\nu)}}{\sqrt{\Phi_{n}^{(\nu)} \Phi_{n}^{(\mu)}}}\right) \\
& =2 \pi\left(\sum_{h=0}^{q} f_{h} R_{h}^{(\nu \mu)}+\sum_{h=-q}^{-1} f_{h} R_{-h}^{(\mu \nu)}\right) . \\
& =2 \pi \sum_{h=-q}^{q} f_{h} R_{-h}^{(\mu \nu)}
\end{aligned}
$$

Hence, we can use the spectral representation of $R_{-h}^{(\mu \nu)}$ as in (4.3.4) in the above limit to get

$$
\begin{aligned}
\lim _{n \rightarrow \infty} D_{n}^{-1} X^{\prime} R X D_{n}^{-1} & =2 \pi \sum_{h=-q}^{q} f_{h} \int_{-\pi}^{\pi} e^{-i h \lambda} d M(\lambda) \\
& =2 \pi \int_{-\pi}^{\pi} \sum_{h=-q}^{q} f_{h} e^{-i h \lambda} d M(\lambda) \\
& =2 \pi \int_{-\pi}^{\pi} f(-\lambda) d M(\lambda)
\end{aligned}
$$

Also we know that

$$
\lim _{n \rightarrow \infty}\left(D_{n}\left(X^{\prime} X\right)^{-1} D_{n}\right)^{-1}=\lim _{n \rightarrow \infty} D_{n}^{-1} X^{\prime} X D_{n}^{-1}=R_{0}=M
$$

We assumed that $M$ is nonsingular, so that

$$
\begin{aligned}
& \lim _{n \rightarrow \infty} D_{n} E(\hat{\beta}-\beta)(\hat{\beta}-\beta)^{\prime} D_{n} \\
= & \lim _{n \rightarrow \infty} D_{n}\left(X^{\prime} X\right)^{-1} X^{\prime} R X\left(X^{\prime} X\right)^{-1} D_{n} \\
= & \lim _{n \rightarrow \infty}\left(D_{n}\left(X^{\prime} X\right)^{-1} D_{n}\right)\left(D_{n}^{-1} X^{\prime} R X D_{n}^{-1}\right)\left(D_{n}\left(X^{\prime} X\right)^{-1} D_{n}\right) \\
= & 2 \pi M^{-1} \int_{-\pi}^{\pi} f(-\lambda) d M(\lambda) M^{-1} .
\end{aligned}
$$

We can extend from $M A(q)$ to a general model of errors, based on the fact that any 
piecewise continuous function can be approximated by trigonometric polynomial functions, i.e. for all $f>0$ (continuous or piecewise continuous) and for all $\delta>0$, there exist $f_{1}$ and $f_{2}$ which are spectral densities of some $M A(q) \mathrm{s}$, such that

$$
f_{1} \leq f \leq f_{2},\left|f_{1}-f_{2}\right|<\delta
$$

(Grenander, U. (1954)). Hence (4.4.2) remain valid.

\section{The Asymptotic Expressions for the Covariance Matrices of BLUE}

Let us consider errors of the autoregressive type, $A R(\alpha)$ of

$$
b_{0} \epsilon_{t}+b_{1} \epsilon_{t+1}+\cdots+b_{\alpha} \epsilon_{t+\alpha}=\eta_{t+\alpha}
$$

with roots of the characteristic equation $\sum_{k=0}^{\alpha} b_{k} z^{k}=0$ outside the unit circle. $\left\{\eta_{1}\right\}$ is white noise with variance one. The spectral density of this model is

$$
f(\lambda)=\frac{1}{2 \pi} \frac{1}{\left|\sum_{\nu=0}^{\alpha} b_{v} e^{i v \lambda}\right|^{2}}
$$

Consider the space spanned by the random variables $\epsilon_{1}, \epsilon_{2}, \ldots, \epsilon_{n}$. Since $\eta_{\alpha+1}, \eta_{\alpha+2}, \ldots, \eta_{n}$ are orthonormal and orthogonal to $\epsilon_{1}, \epsilon_{2}, \ldots, \epsilon_{\alpha}$, we can use the Gram-Schmidt orthogonalization procedure on the vectors. With some weights $d_{v \mu}$, we construct some equations 


$$
\begin{aligned}
& d_{11} \epsilon_{1}=\rho_{1} \\
& d_{21} \epsilon_{1}+d_{22} \epsilon_{2}=\rho_{2} \\
& \text {................ } \\
& d_{\alpha 1} \epsilon_{1}+d_{\alpha 2} \epsilon_{2}+\cdots+d_{\alpha \alpha} \epsilon_{\alpha}=\rho_{\alpha} \\
& b_{0} \epsilon_{1}+b_{1} \epsilon_{2}+\cdots+b_{\alpha} \epsilon_{\alpha+1}=\rho_{\alpha+1}=\eta_{\alpha+1} \\
& 0 \cdot \epsilon_{1}+b_{0} \epsilon_{2}+\cdots+b_{\alpha} \epsilon_{\alpha+2}=\rho_{\alpha+2}=\eta_{\alpha+2} \\
& 0 \cdot \epsilon_{1}+0 \epsilon_{2}+\cdots+b_{0} \epsilon_{n-\alpha}+\cdots+b_{\alpha} \epsilon_{n}=\rho_{n}=\eta_{n}
\end{aligned}
$$

where the $\rho$ 's are orthonormal vectors. The above equations can be written by premultiplying $\epsilon=\left(\begin{array}{c}\epsilon_{1} \\ \vdots \\ \epsilon_{n}\end{array}\right)$ by $\Delta$

$$
\Delta \epsilon=\rho,
$$

where $\Delta$ is an nonsingular transformation. Taking the covariance matrix of both sides of (4.4.4), we have $\Delta R \Delta^{\prime}=I$ so that

$$
R=\Delta^{-1}\left(\Delta^{\prime}\right)^{-1} \Rightarrow R^{-1}=\Delta^{\prime} \Delta
$$

Except $\alpha^{2}$ elements, the others in the matrix $R^{-1}$ are

$$
\left\{R^{-1}\right\}_{v \mu}=\sum_{u=-\infty}^{\infty} \bar{b}_{v+u} b_{\mu+u}
$$

where $b_{v}=0$ if $v<0$ or $v>\alpha$. Next we apply generalized Parseval equality. Let

$$
\begin{aligned}
& g(\lambda)=\frac{1}{\sqrt{2 \pi}} \sum_{v=0}^{\alpha} b_{v} e^{i \nu \lambda}, \text { and } \\
& \qquad x(\lambda)=e^{i \nu \lambda} g(\lambda) \text { and } y(\lambda)=e^{i \mu \lambda} g(\lambda) .
\end{aligned}
$$

Then 


$$
\begin{aligned}
& a_{n}=\left\langle x, e_{n}\right\rangle=\int_{-\pi}^{\pi} e^{i \nu \lambda} g(\lambda) e^{i n \lambda} d \lambda=\int_{-\pi}^{\pi} e^{i(\nu+n) \lambda} g(\lambda) d \lambda, \\
& b_{n}=\left\langle y, e_{n}\right\rangle=\int_{-\pi}^{\pi} e^{i \mu \lambda} g(\lambda) e^{i n \lambda} d \lambda=\int_{-\pi}^{\pi} e^{i(\mu+n) \lambda} g(\lambda) d \lambda .
\end{aligned}
$$

Also $\langle x, y\rangle=\int_{-\pi}^{\pi} x(\lambda) \overline{y(\lambda)} d \lambda=\int_{-\pi}^{\pi} e^{i v \lambda} g(\lambda) e^{-i \mu \lambda} \overline{g(\lambda)} d \lambda=\int_{-\pi}^{\pi} e^{i(v-\mu) \lambda}|g(\lambda)|^{2} d \lambda$. If we have $g(\lambda)=\sum_{k=-\infty}^{\infty} \alpha_{k} e^{i k \lambda}$, then $\alpha_{k}=\int_{-\pi}^{\pi} e^{i k \lambda} g(\lambda) d \lambda$, where $\alpha_{k}$ is the $k$ th coefficient of $g(\lambda)$. Hence $a_{n}=\alpha_{n+v}$. Similarly, we have $b_{n}=\beta_{n+v}$. Therefore, we have

$$
\begin{aligned}
\langle x, y\rangle & =\sum_{n=-\infty}^{\infty} a_{n} \bar{b}_{n}=\sum_{n=-\infty}^{\infty} \alpha_{n+v} \bar{\beta}_{n+\mu}=\sum_{n=-\infty}^{\infty} b_{n+v} \bar{b}_{n+\mu} \\
& =\int_{-\pi}^{\pi} e^{i(v-\mu) \lambda}|g(\lambda)|^{2} d \lambda=\int_{-\pi}^{\pi} e^{i(v-\mu) \lambda} \frac{1}{2 \pi}\left|\sum_{\nu=0}^{\alpha} b_{v} e^{i v \lambda}\right|^{2} d \lambda \\
& =\frac{1}{4 \pi^{2}} \int_{-\pi}^{\pi} e^{i(v-\mu) \lambda} 2 \pi\left|\sum_{\nu=0}^{\alpha} b_{\nu} e^{i v \lambda}\right|^{2} d \lambda=\frac{1}{4 \pi^{2}} \int_{-\pi}^{\pi} e^{i(v-\mu) \lambda} \frac{1}{f(\lambda)} d \lambda .
\end{aligned}
$$

Let $\left\{r_{u, v}^{(-1)}\right\}=R^{-1}$ and $\theta_{m}=\frac{1}{4 \pi^{2}} \int_{-\pi}^{\pi} \frac{e^{i m \lambda}}{f(\lambda)} d \lambda$. Then we have

$$
\begin{aligned}
\left\{D_{n}^{-1} X^{\prime} R^{-1} X D_{n}^{-1}\right\}_{\nu \mu} & =\frac{\sum_{j, k=1}^{n} \bar{X}_{j}^{(\nu)} r_{j, k}^{(-1)} X_{k}^{(\mu)}}{\sqrt{\Phi_{n}^{(v)} \Phi_{n}^{(\mu)}}}, \text { let } j-k=m \\
& =\sum_{m=0}^{\alpha} \theta_{m} \frac{\sum_{k=1}^{n-m} X_{k}^{(\mu)} \bar{X}_{m+k}^{(\nu)}}{\sqrt{\Phi_{n}^{(\nu)} \Phi_{n}^{(\mu)}}}+\sum_{m=-\alpha}^{-1} \theta_{m} \frac{\sum_{j=1}^{n+m} X_{j-m}^{(\mu)} \bar{X}_{j}^{(\nu)}}{\sqrt{\Phi_{n}^{(\nu)} \Phi_{n}^{(\mu)}}}+\delta_{n} .
\end{aligned}
$$

where $\delta_{n}$ is the sum of at most $\alpha^{2}$ terms of the form

$$
r_{j, k}^{(-1)} \frac{\bar{X}_{j}^{(\nu)} X_{k}^{(\mu)}}{\sqrt{\Phi_{n}^{(\nu)} \Phi_{n}^{(\mu)}}}
$$

and $\delta_{n}$ tends to zero as $n \rightarrow \infty$. First, from $R^{-1}=\Delta^{\prime} \Delta$, we can see that $r_{j, k}^{(-1)}$ is bounded uniformly in $n$, 


$$
\left|r_{j, k}^{(-1)}\right| \leq(\alpha+1) G^{2}, \text { where } G^{2}=\max _{0 \leq i, j, k \leq \alpha}\left\{d_{i, j}, b_{k}\right\}
$$

Then

$$
\frac{\left|X_{u+1}^{(u)}\right|^{2}}{\Phi_{n}^{(v)}}=\frac{\Phi_{u+1}^{(v)}-\Phi_{u}^{(v)}}{\Phi_{n}^{(v)}}=\left[\frac{\Phi_{u+1}^{(v)}}{\Phi_{u}^{(v)}}-1\right] \frac{\Phi_{u}^{(v)}}{\Phi_{n}^{(v)}}
$$

If $u$ is bounded, $\frac{\Phi_{u}^{(v)}}{\Phi_{n}^{(v)}}$ tends to zero as $n \rightarrow \infty$. Otherwise, $\frac{\Phi_{u+1}^{(v)}}{\Phi_{u}^{(v)}}$ tends to one by the slowly increasing property of $\Phi_{n}^{(v)}$. By taking the limit of $D_{n}^{-1} X^{\prime} R^{-1} X D_{n}^{-1}$, we have

$$
\begin{aligned}
\lim _{n \rightarrow \infty} D_{n}^{-1} X^{\prime} R^{-1} X D_{n}^{-1} & =\lim _{n \rightarrow \infty}\left(\sum_{m=0}^{\alpha} \theta_{m} \frac{\sum_{k=1}^{n-m} X_{k}^{(\mu)} \bar{X}_{m+k}^{(\nu)}}{\sqrt{\Phi_{n}^{(\nu)} \Phi_{n}^{(\mu)}}}+\sum_{m=-\alpha}^{-1} \theta_{m} \frac{\sum_{j=1}^{n+m} X_{j-m}^{(\mu)} \bar{X}_{j}^{(\nu)}}{\sqrt{\Phi_{n}^{(\nu)} \Phi_{n}^{(\mu)}}}+\delta_{n}\right) \\
& =\sum_{m=0}^{\alpha} \theta_{m} \lim _{n \rightarrow \infty} \frac{\sum_{k=1}^{n-m} X_{k}^{(\mu)} \bar{X}_{m+k}^{(\nu)}}{\sqrt{\Phi_{n}^{(\nu)} \Phi_{n}^{(\mu)}}}+\sum_{m=-\alpha}^{-1} \theta_{m} \lim _{n \rightarrow \infty} \frac{\sum_{j=1}^{n+m} X_{j-m}^{(\mu)} \bar{X}_{j}^{(\nu)}}{\sqrt{\Phi_{n}^{(\nu)} \Phi_{n}^{(\mu)}}} \\
& =\sum_{m=0}^{\alpha} \theta_{m} R_{m}^{(\nu \mu)}+\sum_{m=-\alpha}^{-1} \theta_{m} R_{-m}^{(\nu \mu)}=\sum_{m=-\alpha}^{\alpha} \theta_{m} R_{-m}^{(\nu \mu)}
\end{aligned}
$$

Hence, we can use the spectral representation of $R_{-m}^{(\mu \nu)}$ as in (4.3.4) in the above limit to get

$$
\begin{aligned}
\lim _{n \rightarrow \infty} D_{n}^{-1} X^{\prime} R^{-1} X D_{n}^{-1} & =\sum_{m=-\alpha}^{\alpha} \theta_{m} \int_{-\pi}^{\pi} e^{-i m \lambda} d M(\lambda)=\int_{-\pi}^{\pi} \sum_{m=-\alpha}^{\alpha} \theta_{m} e^{-i m \lambda} d M(\lambda) \\
& =\int_{-\pi}^{\pi} \sum_{m=-\alpha}^{\alpha}\left[\frac{e^{-i m \lambda}}{4 \pi^{2}} \int_{-\pi}^{\pi} \frac{e^{i m \lambda}}{f(\lambda)} d \lambda\right] d M(\lambda) \\
& =\int_{-\pi}^{\pi} \frac{1}{2 \pi} \sum_{m=-\alpha}^{\alpha}\left[\frac{e^{-i m \lambda}}{2 \pi} r(m)\right] d M(\lambda) \\
& =\int_{-\pi}^{\pi} \frac{1}{2 \pi} \frac{1}{f(-\lambda)} d M(\lambda)=\frac{1}{2 \pi} \int_{-\pi}^{\pi} \frac{1}{f(-\lambda)} d M(\lambda) .
\end{aligned}
$$

We can extend from $A R(\alpha)$ to a general model of errors. For all $f>0$ (continuous or piecewise continuous) and for all $\delta>0$, there exist $g_{1}$ and $g_{2}$ which 
are spectral densities of some $A R(\alpha) \mathrm{s}$, such that

$$
g_{1} \leq f \leq g_{2},\left|g_{1}-g_{2}\right|<\delta
$$

(Grenander, U. (1954)). Hence, (4.4.6) is true by approximating it by MA or AR models.

Since

$$
\int_{-\pi}^{\pi} \frac{1}{f(-\lambda)} d M(\lambda) \geq \frac{\int_{-\pi}^{\pi} 1 d M(\lambda)}{\sup _{\lambda} f(-\lambda)}=\frac{M}{\sup _{\lambda} f(-\lambda)}
$$

and $M$ is nonsingular,$\frac{M}{\sup _{\lambda} f(-\lambda)}$ is also nonsingular and then so is $\int_{-\pi}^{\pi} \frac{1}{f(-\lambda)} d M(\lambda)$

$$
\lim _{n \rightarrow \infty} D_{n} E(\tilde{\beta}-\beta)(\tilde{\beta}-\beta)^{\prime} D_{n}=\lim _{n \rightarrow \infty}\left\{D_{n}^{-1} X^{\prime} R^{-1} X D_{n}^{-1}\right\}^{-1}=2 \pi\left\{\int_{-\pi}^{\pi} \frac{1}{f(-\lambda)} d M(\lambda)\right\}^{-1}
$$

Hence from the definition of BLUE, we have

$$
M^{-1} 2 \pi \int_{-\pi}^{\pi} f(-\lambda) d M(\lambda) M^{-1} \geq 2 \pi\left\{\int_{-\pi}^{\pi} \frac{1}{f(-\lambda)} d M(\lambda)\right\}^{-1}
$$

For real-valued case which is of greatest interest, the process $y_{t}$, the regression components $X_{t}^{(j)}$ and the errors $\epsilon_{t}$ are real-valued, such that the spectral density $f(\lambda)=f(-\lambda)$ and $d M(\lambda)=d M(-\lambda)$. Let

$$
T(\lambda)=M(\lambda)-M(-\lambda), \quad 0 \leq \lambda \leq \pi,
$$

where $T(\lambda)$ is a nondecreasing matrix with real elements. Since 


$$
\begin{aligned}
\int_{-\pi}^{\pi} f(\lambda) d M(\lambda) & =\int_{-\pi}^{0} f(\lambda) d M(\lambda)+\int_{0}^{\pi} f(\lambda) d M(\lambda) \\
& =\int_{-\pi}^{0} f(-u) d M(-u)+\int_{0}^{\pi} f(\lambda) d M(\lambda) \\
& =-\int_{0}^{\pi} f(\lambda) d M(-\lambda)+\int_{0}^{\pi} f(\lambda) d M(\lambda) \\
& =\int_{0}^{\pi} f(\lambda) d(M(\lambda)-M(-\lambda))=\int_{0}^{\pi} f(\lambda) d T(\lambda),
\end{aligned}
$$

and similarly,

$$
\int_{-\pi}^{\pi} \frac{1}{f(\lambda)} d M(\lambda)=\int_{0}^{\pi} \frac{1}{f(\lambda)} d T(\lambda)
$$

(4.4.2) and (4.4.7) can be written as

$$
\lim _{n \rightarrow \infty} D_{n} E(\hat{\beta}-\beta)(\hat{\beta}-\beta)^{\prime} D_{n}=T^{-1} 2 \pi \int_{0}^{\pi} f(\lambda) d T(\lambda) T^{-1}
$$

and

$$
\lim _{n \rightarrow \infty} D_{n} E(\tilde{\beta}-\beta)(\tilde{\beta}-\beta)^{\prime} D_{n}=2 \pi\left\{\int_{0}^{\pi} \frac{1}{f(\lambda)} d T(\lambda)\right\}^{-1}
$$

respectively, where $T=T(\pi)=M$.

In the same way as we defined the regression spectrum $S$ in the complex-valued case, using $M$ we can define it using $T$ by saying that $S$ is the set of points $\lambda$ for which there exist $\lambda_{1}, \lambda_{2}: \lambda_{1}<\lambda<\lambda_{2}$ and $\mathrm{T}\left(\lambda_{2}\right)-T\left(\lambda_{1}\right)=\Delta T(I)>0$, where $I=\left(\lambda_{1}, \lambda_{2}\right)$. However, $S$ is defined in the interval $0 \leq \lambda \leq \pi$ instead of $-\pi \leq \lambda \leq \pi$. 


\subsection{Asymptotic Efficiency Conditions of LSE}

Let

$$
N(\lambda)=M^{-1 / 2} M(\lambda) M^{-1 / 2}
$$

For $\lambda_{1}>\lambda_{2}$, we have $M\left(\lambda_{1}\right)-M\left(\lambda_{2}\right) \geq 0$ such that

$$
N\left(\lambda_{1}\right)-N\left(\lambda_{2}\right)=M^{-1 / 2}\left[M\left(\lambda_{1}\right)-M\left(\lambda_{2}\right)\right] M^{-1 / 2} \geq 0
$$

Hence, $N(\lambda)$ is a non-decreasing function. $N(\lambda)$ is normed so that

$$
\begin{aligned}
N(\pi)-N(-\pi) & =M^{-1 / 2}[M(\pi)-M(-\pi)] M^{-1 / 2} \\
& =M^{-1 / 2} M M^{-1 / 2}=I=\text { identity matrix. }
\end{aligned}
$$

If $\int_{H} d N(\lambda)=0$, we say that the set $H$ has zero $N$ measure and we identify sets differing only by a set of zero $N$ measure. We will say that $H=H^{\prime}$ if $N\left(H \Delta H^{\prime}\right)=0$, where $\Delta$ stands for symmetric difference here. We have if $H$ has zero $N$ measure,

$$
0=\int_{H} d N(\lambda)=\int_{H} d M^{-1 / 2} M(\lambda) M^{-1 / 2}=M^{-1 / 2} \int_{H} d M(\lambda) M^{-1 / 2} .
$$

Then we have $\int_{H} d M(\lambda)=0$, i.e. A set is zero $N$ measure if and only if it is zero $M$ measure.

Suppose we can decompose the set $S$ into two sets $E_{\mathrm{I}}$ and $E_{2}$, such that $E_{1} \subset S$ and $E_{2}=S-E_{1}$. If there is no set $E_{1} \subset S$ such that the matrices satisfy

$$
\left\{\begin{array}{l}
N\left(E_{1}\right)=\int_{E_{1}} d N(\lambda), N\left(E_{2}\right)=\int_{E_{2}} d N(\lambda)>0, \\
N\left(E_{1}\right) N\left(E_{2}\right)=0, \text { i.e. orthogonal, }
\end{array}\right.
$$

we say that $S$ cannot be decomposed and it has one element, the set $S$ itself. 
Otherwise, if there are sets $E_{1}$ and $E_{2}$ satisfying the above conditions, we can try to decompose them again in the same way, until the decompositions end in a finite number of steps. As $I$ is of order $p$ (which is finite), there are at almost $p$ elements $E_{i}$ such that:

$$
\left\{\begin{array}{l}
N\left(E_{i}\right)>0 \\
\sum N\left(E_{i}\right)=I \\
N\left(E_{i}\right) N\left(E_{j}\right)=0, i \neq j,
\end{array}\right.
$$

where $N\left(E_{i}\right)>0$ means $N\left(E_{i}\right)$ is a nonnegative definite matrix that is not zero. This is since all $N\left(E_{i}\right)$ are orthogonal projections (If we multiply $\sum N\left(E_{j}\right)=I$ by $N\left(E_{i}\right)$, we have $N\left(E_{i}\right) \sum N\left(E_{j}\right)=N\left(E_{i}\right) I \Rightarrow N^{2}\left(E_{i}\right)=N\left(E_{i}\right)$, i.e., $\quad N\left(E_{i}\right) \quad$ is a projection operator) and there are at most $p$ of such projections in $\mathbb{R}^{p}$.

Let $q$ be the number of elements in the final decomposition, $E_{1}, E_{2}, \cdots, E_{q}, q \leq p$. Note that none of the $E_{i}$ can be decomposed further. This final decomposition is the one will be considered from now on. The $E_{i}$ will be non-overlapping. Then we have

$$
\left\{\begin{array}{l}
N\left(E_{j}\right)=\int_{E_{j}} d N(\lambda)>0, j=1,2, \cdots, q, \\
\sum_{j=1}^{q} N\left(E_{j}\right)=I, \\
N\left(E_{i}\right) N\left(E_{j}\right)=0, \text { for } i \neq j,
\end{array}\right.
$$

Here we show that this maximum decomposition given above defines the elements of $S$ uniquely. Suppose the opposite is true, i.e. there are two different decompositions $A=\left\{E_{j}\right\}$ and $B=\left\{E_{j}^{\prime}\right\}$, which are form maximal decompositions of $S$ into non-overlapping sets with orthogonal nonnegative finite $N$ increments. Therefore, there 
must be one set in $A$ overlapping with two sets in $B$; that is

$$
\left\{\begin{array}{l}
N\left(E_{k} \cap E_{i}^{\prime}\right)>0 \\
N\left(E_{k} \cap E_{j}^{\prime}\right)>0
\end{array} .\right.
$$

We now need the following result to apply in our proof. Suppose $M_{1}, M_{2}, N_{1}, N_{2}$ are Hermitian non-negative definite $p \times p$ matrices, such that

$$
\left\{\begin{array}{l}
M=M_{1}+M_{2}, \\
N=N_{1}+N_{2}, \\
M N=0,
\end{array}\right.
$$

then $M_{i} N_{j}=0, i, j=1,2$.

Apply a unitary transformation to $M$, i.e. $M=U^{\prime} A U$, where $\mathrm{U}$ is a unitary matrix and $\mathrm{A}$ is a diagonal matrix with the first $m$ diagonal elements $d_{1}, d_{2}, \cdots, d_{m}>0$. As we know that $M N=U^{\prime} A U N=0$, we have $A U N U^{\prime}=0$, since $\mathrm{U}$ is invertible and $U^{\prime}=U^{-1}$. We will use the following results: We have a $p \times p$ matrix $H$ which is nonnegative definite and a diagonal matrix $\Delta$ with the first $m$ diagonal elements $d_{1}, d_{2}, \cdots, d_{m}>0$. We know that the product of them is zero:

$$
\Delta H=0 \Rightarrow\left[\begin{array}{cc}
\Delta & 0 \\
0 & 0
\end{array}\right]\left[\begin{array}{ll}
A & B \\
C & D
\end{array}\right]=\left[\begin{array}{cc}
\Delta A & \Delta B \\
0 & 0
\end{array}\right]=0
$$

Since $\Delta$ is a diagonal matrix with nonzero elements $d_{1}, d_{2}, \cdots, d_{m}$ which is invertible, we see that $\Delta^{-1} \Delta A=0$ and $\Delta^{-1} \Delta B=0$ and then $A$ and $B$ are both zero matrices. On using the nonnegative definiteness of the matrices, we see that the only non-zero elements of $H$ are those with subscripts $(r, s)$ where $m+1 \leq r, s \leq p$, i.e. 


$$
H=\left[\begin{array}{ll}
0 & 0 \\
0 & D
\end{array}\right]
$$

As we mentioned before, $A U N U^{\prime}=0$. Given that $N \geq 0$. Since $\left(\alpha^{\prime} U\right) N\left(U^{\prime} \alpha\right) \geq 0$ for any nonzero vector $\alpha, U N U^{\prime}$ is a nonnegative definite matrix. Hence, by previous result, $U N U^{\prime}$ is in the same form of $H$ which has non-zero elements with subscripts $(r, s)$ where $m+1 \leq r, s \leq p$.

We know that $N_{1}$ and $N_{2}$ are nonnegative definite matrices, so the diagonal elements of $U N_{1} U^{\prime}$ and $U N_{2} U^{\prime}$ should be nonnegative. Since

$$
U N U^{\prime}=U\left(N_{1}+N_{2}\right) U^{\prime}=U N_{1} U^{\prime}+U N_{2} U^{\prime}=\left[\begin{array}{ll}
0 & 0 \\
0 & K
\end{array}\right]
$$

where $K$ is some non-zero matrix, we can say that $U N_{1} U^{\prime}$ and $U N_{2} U^{\prime}$ are in the same form of $U N U^{\prime}$ and hence $A U N_{1} U^{\prime}=A U N_{2} U^{\prime}=0$. Therefore,

$$
\left\{\begin{array} { l } 
{ U M U ^ { \prime } U N _ { 1 } U ^ { \prime } = 0 } \\
{ U M U ^ { \prime } U N _ { 2 } U ^ { \prime } = 0 }
\end{array} \Rightarrow \left\{\begin{array}{l}
M N_{1}=0 \\
M N_{2}=0
\end{array}\right.\right.
$$

Next, we will apply the same idea as above to $M N_{1}=0$. We can use a unitary transformation to $N_{1}$ to carry it into diagonal form with the diagonal matrix $B_{1}$ with the last $n$ diagonal elements greater than zero, i.e. $M V^{\prime} B_{1} V=0$. Then we have $V M V^{\prime} B=0$, such that the only non-zero elements of $V M V^{\prime}$ are those with subscripts $(r, s)$ where $1 \leq r, s \leq p-n$. The same is clearly true of $V M_{1} V^{\prime}$ and $V M_{2} V^{\prime}$, since $M_{1}, M_{2}$ are nonnegative definite and $B M B^{\prime}=B M_{1} B^{\prime}+B M_{2} B^{\prime}$. Hence we get

$$
\left\{\begin{array} { l } 
{ B M _ { 1 } B ^ { \prime } B N _ { 1 } B ^ { \prime } = 0 } \\
{ B M _ { 2 } B ^ { \prime } B N _ { 1 } B ^ { \prime } = 0 }
\end{array} \Rightarrow \left\{\begin{array}{l}
M_{1} N_{1}=0 \\
M_{2} N_{1}=0
\end{array} .\right.\right.
$$


Similarly, we can use the same idea to $M N_{2}=0$ to get

$$
\left\{\begin{array}{l}
M_{1} N_{2}=0 \\
M_{2} N_{2}=0
\end{array}\right. \text {. }
$$

Therefore, we have proved that $M_{i} N_{j}=0, i, j=1,2$.

We can write $E_{k}=\left(E_{i} \cap E_{k}\right) \bigcup F=D \bigcup F$. Then $N\left(E_{k}\right)=N(D)+N(F)$ is orthogonal to every $N\left(E_{l}\right)$, for $l \neq k$. By the above result, $N(D)$ and $N(F)$ are also orthogonal to every $N\left(E_{l}\right)$, for $l \neq k$. Since we know that $N\left(E_{i}^{\prime}\right) N\left(\bigcup_{j \neq i} E_{j}^{\prime}\right)=0$, also by the above result it follows that $N\left(E_{i}^{\prime} \cap E_{k}\right) N(F)=0$, so that $A=\left\{E_{j}\right\}$ could be decomposed further, which is contrary to our assumption that $A=\left\{E_{j}\right\}$ is the final decomposition. Hence, we proved the uniqueness of the decomposition above.

As we mentioned in (4.3.9), that $\hat{\beta}$ is asymptotically efficient if

$$
\lim _{n \rightarrow \infty} D_{n} E(\hat{\beta}-\beta)(\hat{\beta}-\beta)^{\prime} D_{n}=\lim _{n \rightarrow \infty} D_{n} E(\tilde{\beta}-\beta)(\tilde{\beta}-\beta)^{\prime} D_{n} .
$$

Following the results we got in section 4.4 , a necessary and sufficient condition for the asymptotic efficiency of the least square estimator is that

$$
\begin{aligned}
& M^{-1} 2 \pi \int_{-\pi}^{\pi} f(-\lambda) d M(\lambda) M^{-1}=2 \pi\left\{\int_{-\pi}^{\pi} \frac{1}{f(-\mu)} d M(\mu)\right\}^{-1} \\
\Leftrightarrow & M^{-1} \int_{-\pi}^{\pi} f(-\lambda) d M(\lambda) M^{-1}=\left\{\int_{-\pi}^{\pi} \frac{1}{f(-\mu)} d M(\mu)\right\}^{-1} \\
\Leftrightarrow & M^{-1 / 2} \int_{-\pi}^{\pi} f(-\lambda) d M(\lambda) M^{-1 / 2}=M^{1 / 2}\left\{\int_{-\pi}^{\pi} \frac{1}{f(-\mu)} d M(\mu)\right\}^{-1} M^{1 / 2} \\
\Leftrightarrow & \int_{-\pi}^{\pi} f(-\lambda) M^{-1 / 2} d M(\lambda) M^{-1 / 2}=\left\{\int_{-\pi}^{\pi} \frac{1}{f(-\mu)} M^{-1 / 2} d M(\mu) M^{-1 / 2}\right\}^{-1} \\
\Leftrightarrow & \int_{-\pi}^{\pi} f(-\lambda) d N(\lambda)=\left\{\int_{-\pi}^{\pi} \frac{1}{f(-\mu)} d N(\mu)\right\}^{-1} \\
\Leftrightarrow & \int_{-\pi}^{\pi} f(-\lambda) d N(\lambda) \int_{-\pi}^{\pi} \frac{1}{f(-\mu)} d N(\mu)=I .
\end{aligned}
$$


According to the decomposition of $S$ into elements, we have (4.5.1) equivalent to

$$
\sum_{j=1}^{q} \int_{E_{j}} f(-\lambda) d N(\lambda) \int_{E_{j}} \frac{1}{f(-\mu)} d N(\mu)=I
$$

If $f(-\lambda)=c_{j}>0$ on $E_{j}$, then we need

$$
\begin{aligned}
& \sum_{j=1}^{q} \int_{E_{j}} c_{j} d N(\lambda) \int_{E_{j}} \frac{1}{c_{j}} d N(\mu)=I \\
& \Leftrightarrow \sum_{j=1}^{q} \int_{E_{j}} d N(\lambda) \int_{E_{i}} d N(\mu)=I \\
& \Leftrightarrow \sum_{j=1}^{q} N^{2}\left(E_{j}\right)=\sum_{j=1}^{q} N\left(E_{j}\right)=I
\end{aligned}
$$

so that we have asymptotic efficiency under this condition.

We are going to prove the converse is true as well. First, let us assume

$$
\int_{-\pi}^{\pi} f(-\lambda) d N(\lambda) \int_{-\pi}^{\pi} \frac{1}{f(-\mu)} d N(\mu)=I
$$

Let $U$ be an orthogonal matrix that satisfies

$$
N(\lambda)=U^{\prime} A(\lambda) U
$$

where $A(\lambda)$ is a diagonal matrix. So

$$
A(\lambda)=U N(\lambda) U^{\prime}
$$

From (4.5.2), we have

$$
\int_{-\pi}^{\pi} f(-\lambda) d\left\{U N(\lambda) U^{\prime}\right\}_{i i} \int_{-\pi}^{\pi} \frac{1}{f(-\mu)} d\left\{U N(\mu) U^{\prime}\right\}_{i i}=1, i=1,2, \cdots, p
$$

By Schwarz inequality applied with $g_{1}(\lambda)=\sqrt{f(\lambda)}$ and $g_{2}(\lambda)=1 / \sqrt{f(\lambda)}$, we have 


$$
\begin{aligned}
1 & =\int_{-\pi}^{\pi} 1 d\left\{U N(\lambda) U^{\prime}\right\}_{" i} \\
& =\int_{-\pi}^{\pi} g_{1}(\lambda) g_{2}(\lambda) d\left\{U N(\lambda) U^{\prime}\right\}_{i i} \\
& \leq \int_{-\pi}^{\pi}\left|g_{1}(\lambda)\right|^{2} d\left\{U N(\lambda) U^{\prime}\right\}_{i i} \int_{-\pi}^{\pi}\left|g_{2}(\lambda)\right|^{2} d\left\{U N(\lambda) U^{\prime}\right\}_{i i} \cdot \\
& =\int_{-\pi}^{\pi} f(-\lambda) d\left\{U N(\lambda) U^{\prime}\right\}_{i i} \int_{-\pi}^{\pi} \frac{1}{f(-\mu)} d\left\{U N(\mu) U^{\prime}\right\}_{i i}
\end{aligned}
$$

The equality holds if and only if the integrands are proportional, on the set on which the non-decreasing function $N_{i}(\lambda)=\left\{U N(\lambda) U^{\prime}\right\}_{i i}$ increases, i.e.

$$
g_{1}(\lambda)=k g_{2}(\lambda) \Rightarrow k \sqrt{f(-\lambda)}=\frac{1}{\sqrt{f(-\lambda)}} \Rightarrow f(-\lambda)=\frac{1}{k}
$$

where $k$ is some constant. For $i=1,2, \cdots, p, f(-\lambda)$ must be a constant $c_{\text {; }}$ on each of these $q$ sets $A_{i}$. Consider any chain of sets $A_{1}, A_{i_{1}}, \cdots, A_{t_{k}}$ such that

$$
\begin{aligned}
& N_{1}\left(A_{1} \bigcap A_{i_{1}}\right)>0 \\
& N_{i_{1}}\left(A_{i_{1}} \bigcap A_{i_{2}}\right)>0 \\
& \cdots \\
& N_{i_{q-1}}\left(A_{i_{k-1}} \bigcap A_{i_{q}}\right)>0 .
\end{aligned}
$$

Hence, $f(-\lambda)$ must equal the same constant $c_{1}$ on every set in the chain. Then $f(-\lambda)=c_{1}$ should be on the union of all sets $A_{i}$ which are in the chain containing the set $A_{1}$. We call the union of these sets $B_{1}$. Similarly, we can construct the non-overlapping set $B_{i}$, for $i=1,2, \cdots, r \leq p$ in the same way by finding the chain sets of $A_{i}$ and $f(-\lambda)=c_{i}$ is a constant on each of these sets.

Now we can write $(4.5 .2)$ in the form that

$$
\sum_{i=1}^{r} N^{2}\left(B_{i}\right)=I
$$


In addition, as we know that $N\left(B_{i}\right)>0$ and

$$
\int_{-\pi}^{\pi} d N(\lambda)=N(\pi)-N(-\pi)=I \Rightarrow \sum_{i=1}^{r} N\left(B_{i}\right)=I,
$$

we have $N\left(B_{i}\right) \leq I$ and $N^{2}\left(B_{i}\right) \leq N\left(B_{i}\right)$. Also, we can get the following equation from (4.5.3),

$$
\begin{aligned}
N^{2}\left(B_{i}\right)-N\left(B_{i}\right) & =\left(I-\sum_{j \neq i} N^{2}\left(B_{j}\right)\right)-\left(I-\sum_{j \neq i} N\left(B_{j}\right)\right) \\
& =\sum_{j \neq i} N\left(B_{j}\right)-\sum_{j \neq i} N^{2}\left(B_{j}\right) \\
& =\sum_{j \neq i}\left(N\left(B_{j}\right)-N^{2}\left(B_{j}\right)\right) \geq 0
\end{aligned}
$$

Hence we conclude that $N^{2}\left(B_{i}\right)=N\left(B_{i}\right)$, i.e., the $N\left(B_{i}\right)$ are projection operators. According to the theorem on page 74 of Halmos (1958), the sum of $N\left(B_{i}\right)$ 's being the identity matrix, which is also a projection operator, then $N\left(B_{i}\right)$ 's must be orthogonal, and each $B_{1}$ is the union of a number of elements of the spectrum $S$. Hence, we have proved that $f(-\lambda)$ is constant on each element.

\subsection{Polynomial and Trigonometric Regression}

Here we will see that for this type of design, the LSE will be asymptotically efficiency for all types of positive and piecewise continuous spectral densities. Let us consider a multiple polynomial and trigonometric regression case with regression variables 


$$
\begin{aligned}
& X_{1}^{(v)}=t^{v} e^{i \lambda_{1}}, v=0,1,2, \cdots, p_{1} \\
& X_{1}^{\left(p_{1}+v^{+}+1\right)}=t^{v} e^{i t \lambda_{2}}, v=0,1,2, \cdots, p_{2} \\
& \left.\begin{array}{l}
\cdots \ldots \ldots \ldots \ldots \ldots \ldots \ldots \ldots \ldots \ldots \ldots . . . \cdots \\
X_{1}^{\left(p_{1}+p_{2}+\cdots+p_{m-1}+m-1+v\right)}=t^{\nu} e^{i\left(\lambda_{m}\right.}, v=0,1,2, \cdots, p_{m}
\end{array}\right\},
\end{aligned}
$$

where $\lambda_{1}, \lambda_{2}, \cdots, \lambda_{m}$ are different frequencies in $(-\pi, \pi)$. Hence we have

$$
\begin{aligned}
\Phi_{n}^{(k)} & =\sum_{t=1}^{n}\left|t^{v} e^{i t \lambda_{s}}\right|^{2}=\sum_{i=1}^{n} t^{2 v} \\
& \approx \int_{t=1}^{n} t^{2 v} d t \text { as } n \rightarrow \infty \\
& =\left.\frac{t^{2 v+1}}{2 v+1}\right|_{t=1} ^{n}=\frac{n^{2 v+1}}{2 v+1}-\frac{1}{2 v+1} \\
& \approx \frac{n^{2 v+1}}{2 v+1}, \text { as } n \rightarrow \infty
\end{aligned}
$$

where the superscript $k$ corresponds to the pair of integers $(v, s), v$ is the power of $t$ and $s$ is the index of the frequency. We can also see that $\Phi_{n}^{(k)}$ is a slowly increasing sequence by the following:

$$
\begin{aligned}
\lim _{n \rightarrow \infty} \frac{\Phi_{n+h}^{(k)}}{\Phi_{n}^{(k)}} & =\lim _{n \rightarrow \infty} \frac{(n+h)^{2 v+1}}{2 v+1} \frac{2 v+1}{n^{2 v+1}} \\
& =\lim _{n \rightarrow \infty} \frac{(n+h)^{2 v+1}}{n^{2 v+1}}=\lim _{n \rightarrow \infty}\left(\frac{n+h}{n}\right)^{2 v+1}=1 .
\end{aligned}
$$

Then according to (4.3.3), let us consider 


$$
\begin{aligned}
& R_{h}^{(k, k)}=\lim _{n \rightarrow \infty} \frac{\sum_{t=1}^{n} X_{t+h}^{(v)} \bar{X}_{t}^{\left(v^{\prime}\right)}}{\sqrt{\Phi_{n}^{(k)} \Phi_{n}^{\left(k^{\prime}\right)}}}=\lim _{n \rightarrow \infty} \frac{\sum_{i=1}^{n}(t+h)^{v} e^{i(t+h) \lambda_{s}} t^{v^{\prime}} e^{-i t \lambda_{s}}}{\sqrt{\Phi_{n}^{(k)} \Phi_{n}^{\left(k^{\prime}\right)}}} \\
& =\lim _{n \rightarrow \infty} \frac{\sum_{t=1}^{n} t^{\nu+v^{\prime}}\left(1+\frac{h}{t}\right)^{v} e^{i(l+h) \lambda_{s}} e^{-i t \lambda_{s^{\prime}}}}{\sqrt{\Phi_{n}^{(k)} \Phi_{n}^{(k)}}} \\
& \approx \lim _{n \rightarrow \infty} \frac{\sum_{t=1}^{n} t^{\nu+v^{\prime}}\left(1+v \frac{h}{t}\right) e^{i(t+h) \lambda_{s}} e^{-i t \lambda_{s^{\prime}}}}{\sqrt{\frac{n^{2 v+1}}{2 v+1} \cdot \frac{n^{2 v^{\prime}+1}}{2 v^{\prime}+1}}} \\
& \approx \lim _{n \rightarrow \infty} \frac{\sum_{t=1}^{n} t^{\nu+\nu^{\prime}} e^{i(t+h) \lambda_{s}} e^{-i t \lambda_{s^{\prime}}}+o\left(\sum_{i=1}^{n} t^{\nu+v^{\prime}} e^{i(t+h) \lambda_{s}} e^{-i t \lambda_{s^{\prime}}}\right)}{\sqrt{\frac{n^{2 v+1}}{2 v+1} \cdot \frac{n^{2 v^{\prime}+1}}{2 v^{\prime}+1}}} \\
& \approx \lim _{n \rightarrow \infty} \frac{\sqrt{(2 v+1)\left(2 v^{\prime}+1\right)}}{n^{v+v^{\prime}+1}} \sum_{i=1}^{n} t^{v+v^{\prime}} e^{i\left(\lambda_{s}-\lambda_{s^{\prime}}\right)} e^{i h \lambda_{s^{\prime}}}
\end{aligned}
$$

If $\lambda_{s}=\lambda_{s}$, we have

$$
\begin{aligned}
R_{h}^{\left(k, k^{\prime}\right)} & =\lim _{n \rightarrow \infty} \frac{\sqrt{(2 v+1)\left(2 v^{\prime}+1\right)}}{n^{i+v^{\prime}+1}} \sum_{i=1}^{n} t^{v+v^{\prime}} e^{i h \lambda_{s^{\prime}}} \\
& \approx \frac{\sqrt{(2 v+1)\left(2 v^{\prime}+1\right)}}{n^{v+v^{\prime}+1}} e^{i h \lambda_{s^{\prime}}} \frac{n^{v^{\prime+v^{\prime}+1}}}{v+v^{\prime}+1} \\
& =\frac{\sqrt{(2 v+1)\left(2 v^{\prime}+1\right)}}{v+v^{\prime}+1} e^{i h \lambda_{s^{\prime}}} .
\end{aligned}
$$

If $\lambda_{s} \neq \lambda_{s^{\prime}}$, we have 


$$
\begin{aligned}
R_{h}^{\left(k, k^{\prime}\right)} & =\lim _{n \rightarrow \infty} \frac{\sqrt{(2 v+1)\left(2 v^{\prime}+1\right)}}{n^{v+v^{\prime}+1}} \sum_{t=1}^{n} t^{\nu+v^{\prime}} e^{i t\left(\lambda_{s}-\lambda_{s^{\prime}}\right)} e^{i h \lambda_{s^{\prime}} \cdot} \\
& \approx \lim _{n \rightarrow \infty} \frac{\sqrt{(2 v+1)\left(2 v^{\prime}+1\right)}}{n^{\nu+v^{\prime}+1}} e^{i h \lambda_{s^{\prime}}} \sum_{t=1}^{n} t^{\nu+v^{\prime}} e^{i\left(\lambda_{s}-\lambda_{s^{\prime}}\right)} \\
& =\lim _{n \rightarrow \infty} \frac{\sqrt{(2 v+1)\left(2 v^{\prime}+1\right)}}{n^{v+v^{\prime}+1}} e^{i h \lambda_{s^{\prime}}} \int_{t=1}^{n} t^{v+v^{\prime}} e^{i t\left(\lambda_{s}-\lambda_{s^{\prime}}\right)} d t \\
& =\lim _{n \rightarrow \infty} \frac{\sqrt{(2 v+1)\left(2 v^{\prime}+1\right)}}{n^{v+v^{\prime}+1}} e^{i h \lambda_{s^{\prime}}} \cdot\left(\left.\frac{t^{v+v^{\prime}} e^{i t\left(\lambda_{s}-\lambda_{s^{\prime}}\right)}}{i\left(\lambda_{s}-\lambda_{s^{\prime}}\right)}\right|_{t=1} ^{n}-\int_{t=1}^{n} \frac{t^{v^{+v^{\prime}-1}} e^{i t\left(\lambda_{s}-\lambda_{s^{\prime}}\right)}}{i\left(\lambda_{s}-\lambda_{s^{\prime}}\right)} d t\right) \\
& =\lim _{n \rightarrow \infty} \frac{\sqrt{(2 v+1)\left(2 v^{\prime}+1\right)}}{n^{v+v^{\prime}+1}} e^{i h \lambda_{s^{\prime}}} O\left(n^{v+v^{\prime}}\right)=0 .
\end{aligned}
$$

Hence we conclude that

$$
R_{h}^{\left(k, k^{\prime}\right)}=\frac{\sqrt{(2 v+1)\left(2 v^{\prime}+1\right)}}{v+v^{\prime}+1} \delta_{s s^{\prime}} e^{i h \lambda_{s^{\prime}}}, \text { where } \delta_{s s^{\prime}}=\left\{\begin{array}{l}
1, \text { if } s=s^{\prime} \\
0, \text { if } s \neq s^{\prime}
\end{array}\right.
$$

from the above results. For the spectral representation

$$
R_{h}=\int_{-\pi}^{\pi} e^{i h \lambda} d M(\lambda)
$$

the dimension of $R_{h}$ is $p \times p$, where $p=\left(p_{1}+1\right)+\left(p_{2}+1\right)+\cdots+\left(p_{m}+1\right)$

$=p_{1}+p_{2}+\cdots+p_{m}+m$. The entries of $R_{h}$ are $R_{h}\left(j, j^{\prime}\right)$, where

$$
\begin{aligned}
& j=p_{1}+p_{2}+\cdots+p_{s-1}+s-1+v, s \leq m, v=0,1, \cdots, p_{s} \\
& j^{\prime}=p_{1}+p_{2}+\cdots+p_{s^{\prime}-1}+s^{\prime}-1+v^{\prime}, s^{\prime} \leq m, v^{\prime}=0,1, \cdots, p_{s^{\prime}} .
\end{aligned}
$$

If $s \neq s^{\prime}$, we have

$$
R_{h}\left(j, j^{\prime}\right)=0
$$

and if $s=s^{\prime}$, we have

$$
R_{h}\left(j, j^{\prime}\right)=\frac{\sqrt{(2 v+1)\left(2 v^{\prime}+1\right)}}{v+v^{\prime}+1} e^{i h \lambda_{s}}
$$

Let $M_{s}$ be the $\left(p_{s}+1\right) \times\left(p_{s}+1\right)$ matrix 


$$
M_{s}=\left\{\frac{\sqrt{(2 v+1)\left(2 v^{\prime}+1\right)}}{v+v^{\prime}+1} ; v, v^{\prime}=0,1, \cdots, p_{s}\right\} .
$$

Then

$$
R_{h}=M_{1} e^{i h \lambda_{1}} \oplus M_{2} e^{i h \lambda_{2}} \oplus \cdots \oplus M_{m} e^{i h \lambda_{m}},
$$

the direct sum of the matrices $M_{1} e^{i h \lambda_{1}}, M_{2} e^{i h \lambda_{2}}, \cdots, M_{m} e^{i h \lambda_{m}}$,

$$
R_{h}=\left[\begin{array}{ccccc}
\overbrace{M_{1} e^{i h \lambda_{1}}}^{p_{1}+1} & 0 & \cdots & \cdots & 0 \\
0 & M_{2} e^{i h \lambda_{2}} & 0 & \cdots & 0 \\
\vdots & & \ddots & & \vdots \\
\vdots & & & \ddots & 0 \\
0 & \cdots & \cdots & 0 & \underbrace{M_{m} e^{i h \lambda_{m}}}_{p_{m}+1}
\end{array}\right] .
$$

From the spectral representation of $R_{h}$, we have

$$
\begin{aligned}
\int_{-\pi}^{\pi} e^{i h \lambda} d M(\lambda) & =R_{h} \\
& =M_{1} e^{i h \lambda_{1}} \oplus M_{2} e^{i h \lambda_{2}} \oplus \cdots \oplus M_{m} e^{i h \lambda_{m}} \\
& =\sum_{s=1}^{m} e^{i h \lambda_{s}} \Delta M\left(\lambda_{s}\right),
\end{aligned}
$$

where

$$
\begin{gathered}
\Delta M\left(\lambda_{1}\right)=\left[\begin{array}{cccc}
M_{1} & 0 & \cdots & 0 \\
0 & 0 & & \vdots \\
\vdots & & \ddots & \vdots \\
0 & \cdots & \cdots & 0
\end{array}\right], \\
\Delta M\left(\lambda_{2}\right)=\left[\begin{array}{cccc}
0 & 0 & \cdots & 0 \\
0 & M_{2} & & \vdots \\
\vdots & & \ddots & \vdots \\
0 & \cdots & \cdots & 0
\end{array}\right],
\end{gathered}
$$




$$
\Delta M\left(\lambda_{m}\right)=\left[\begin{array}{cccc}
0 & \cdots & & 0 \\
\vdots & \ddots & & \vdots \\
\vdots & & 0 & 0 \\
0 & \cdots & 0 & M_{m}
\end{array}\right]
$$

This indicates that $M(\lambda)$ has jumps only at $\lambda_{s}$, where $s=1,2, \cdots, m$. Thus, taking $h=0, M=M(\pi)-M(-\pi)$ is the direct sum of the nonsingular matrices $M_{1}, M_{2}, \cdots, M_{m}$. Since $M_{1}, M_{2}, \cdots, M_{m}$ are all nonsingular matrices (as we will see below), it follows that $M=R_{0}$ is also nonsingular. We now prove that $M_{s}$ is nonsingular. First, we write

$$
M_{s}=D\left\{\frac{1}{v+v^{\prime}+1} ; v, v^{\prime}=0,1, \cdots, p_{s}\right\} D=D A_{s} D
$$

where $D$ is a nonsingular diagonal matrix with the diagonal elements $\sqrt{(2 v+1)}, v=0,1, \cdots, p_{s}$. Suppose that $A_{s}$ were singular, then there would exist a non-zero $\left(p_{s}+1\right) \times 1$ vector $z$ such that

$$
\begin{aligned}
0 & =z^{\prime} A_{s} z=\sum_{v, v^{\prime}=0}^{p_{s}} \bar{z}_{v} z_{v^{\prime}} \frac{1}{v+v^{\prime}+1}=\sum_{v, v^{\prime}=0}^{p_{s}} \bar{z}_{v} z_{v^{\prime}} \cdot \int_{0}^{1} x^{v+v^{\prime}} d x \\
& =\int_{0}^{1} \sum_{v, v^{\prime}=0}^{p_{s}} \bar{z}_{v} z_{v^{\prime}} x^{v+v^{\prime}} d x=\int_{0}^{1}\left|\sum_{v=0}^{p_{s}} z_{v} x^{v^{2}}\right|^{2} d x
\end{aligned}
$$

which implies that

$$
\sum_{v=0}^{p_{s}} z_{v} x^{v} \equiv 0, \text { for all } x \text { in }[0,1]
$$

but then $z_{v}=0$, for all $v$ which contradicts the assumption.

The regression spectrum $S$ of $M(\lambda)$ consists merely of the jump points $\lambda_{1}, \lambda_{2}, \cdots, \lambda_{m}$. Of course any spectral density (of errors) being constant on each point $\lambda_{s}$, 
the least square estimator $\hat{\beta}$ of the regression coefficients is asymptotically efficient relative to BLUE in this context, regardless of the spectral density, provided it is piecewise continuous and positive. There are two simplest particular cases of the previous context.

\section{Example 1: Trigonometric Regression}

$$
y_{t}=\beta_{1} e^{i t \lambda_{1}}+\beta_{2} e^{i \lambda_{2}}+\cdots+\beta_{p} e^{i t \lambda_{p}}+\epsilon_{t}
$$

Applying the above results, if the frequencies $\lambda_{1}, \lambda_{2}, \cdots, \lambda_{p}$ are all different, the least square estimators of $\beta_{1}, \beta_{2}, \cdots, \beta_{p}$ are asymptotically efficient. According to (4.4.2), the asymptotic covariances of these estimators (normalized) will be

$$
\left\{\operatorname{Cov}\left(D_{n} \hat{\beta}_{n}\right)\right\}_{j, k}=\left\{M^{-1} 2 \pi \int_{-\pi}^{\pi} f(-\lambda) d M(\lambda) M^{-1}\right\}_{j, k} .
$$

We have

$$
\Phi_{n}^{(v)}=\sum_{i=1}^{n}\left|X_{t}^{\left({ }^{(n)}\right.}\right|^{2}=\sum_{i=1}^{n}\left|e^{i i \lambda}\right|^{2}=n,
$$

and

$$
D_{n}=\operatorname{diag}\left(n^{\frac{1}{2}}, n^{\frac{1}{2}}, \ldots, n^{\frac{1}{2}}\right)
$$

Then from (4.6.1), we get $M_{s}=1$ as $p_{s}=0$ and $v=v^{\prime}=0$, and hence

$$
\begin{aligned}
M & =M_{1} \oplus M_{2} \oplus \cdots \oplus M_{p} \\
& =I_{p} .
\end{aligned}
$$

Also, we have 


$$
\begin{aligned}
\int_{\pi}^{\pi} f(-\lambda) d M(\lambda) & =\sum_{s=1}^{p} f\left(-\lambda_{s}\right) \Delta M\left(\lambda_{s}\right) \\
& =\operatorname{diag}\left(f\left(-\lambda_{1}\right), f\left(-\lambda_{2}\right), \cdots f\left(-\lambda_{p}\right)\right) .
\end{aligned}
$$

All above results imply that

$$
\left\{\operatorname{Cov}\left(D_{n} \hat{\beta}_{n}\right)\right\}_{j, k} \sim 2 \pi f\left(-\lambda_{j}\right) \delta_{j, k}
$$

Hence, they are asymptotically uncorrelated with variances depending on the spectral density at the points $\lambda_{1}, \lambda_{2}, \cdots, \lambda_{p}$.

\section{Example 2: Polynomial Regression}

$$
y_{t}=\beta_{1} t+\cdots+\beta_{p} t^{p}+\epsilon_{t}
$$

There is only one frequency $\lambda=0$, and the corresponding jump of $M(\lambda)$ is

$$
\Delta M(0)=\left\{\frac{\sqrt{(2 v+1)\left(2 v^{\prime}+1\right)}}{v+v^{\prime}+1} ; v, v^{\prime}=0,1, \cdots, p\right\} .
$$

By applying the main result of this section, the least square estimators are asymptotically

efficient. The covariances are

$$
\left\{\operatorname{Cov}\left(D_{n} \hat{\beta}_{n}\right)\right\}_{j, k} \sim\left\{M^{-1} 2 \pi \int_{-\pi}^{\pi} f(-\lambda) d M(\lambda) M^{-1}\right\}_{j, k} .
$$

As we know that $\Phi_{n}^{(k)}=\sum_{t=1}^{n}\left|t^{\nu}\right|^{2}=\sum_{t=1}^{n} t^{2 \nu} \sim \frac{n^{2 v+1}}{2 v+1}$, we have

$$
D_{n}=\operatorname{diag}\left(\sqrt{\frac{n^{2 v+1}}{2 v+1}}, v=0,1, \cdots, p\right) \text {. }
$$

As

$$
M=\left\{\frac{\sqrt{(2 v+1)\left(2 v^{\prime}+1\right)}}{v+v^{\prime}+1} ; v, v^{\prime}=0,1, \cdots, p\right\},
$$


we have $M=\Delta M(0)$.

Also, since there is only one frequency, we have

$$
\int_{-\pi}^{\pi} f(-\lambda) d M(\lambda)=f(0) \Delta M(0)=f(0) M
$$

Hence, the covariance is

$$
\begin{aligned}
& \left\{\operatorname{Cov}\left(D_{n} \hat{\beta}_{n}\right)\right\}_{j, k} \sim\left\{M^{-1} 2 \pi \int_{-\pi}^{\pi} f(-\lambda) d M(\lambda) M^{-1}\right\}_{j, k} \\
& =\left\{2 \pi f(0) M^{-1}\right\}_{j, k} \\
& =2 \pi f(0)\left\{\frac{\sqrt{(2 v+1)\left(2 v^{\prime}+1\right)}}{v+v^{\prime}+1} ; v, v^{\prime}=0,1, \cdots, p\right\}_{j, k}^{-1} \\
& =2 \pi f(0)\left\{\operatorname{diag}(\sqrt{(2 v+1)}, v=0,1, \cdots, p)\left\{\frac{1}{v+v^{\prime}+1} ; v, v^{\prime}=0,1, \cdots, p\right\} \operatorname{diag}(\sqrt{(2 v+1)}, v=0,1, \cdots, p)\right\}_{j, k}^{-1} \\
& =2 \pi f(0)\left\{\{\operatorname{diag}(\sqrt{(2 v+1)}, v=0,1, \cdots, p)\}^{-1}\left\{\frac{1}{v+v^{\prime}+1} ; v, v^{\prime}=0,1, \cdots, p\right\}^{-1}\{\operatorname{diag}(\sqrt{(2 v+1)}, v=0,1, \cdots, p)\}^{-1}\right\}_{j, k} \\
& =2 \pi f(0)\left\{\operatorname{diag}\left((2 v+1)^{-\frac{1}{2}}, v=0,1, \cdots, p\right)\left\{\frac{1}{v+v^{\prime}+1} ; v, v^{\prime}=0,1, \cdots, p\right\}^{-1} \operatorname{diag}\left((2 v+1)^{-\frac{1}{2}}, v=0,1, \cdots, p\right)\right\}_{j, k} \\
& =2 \pi f(0)(2 j+1)^{-\frac{1}{2}}(2 k+1)^{-\frac{1}{2}}\left\{\frac{1}{\nu+v^{\prime}+1} ; v, v^{\prime}=0,1, \cdots, p\right\}_{j, k}^{-1} \text {. }
\end{aligned}
$$

So we can say that in this context, the (normalized) estimator of $\beta_{1}, \beta_{2}, \cdots, \beta_{p}$ are still correlated (efficient asymptotically) unlike the trigonometric design case. 


\section{M-Estimation of Linear Regression with Dependent Errors}

\subsection{Introduction}

Wu (2007) studied the asymptotic properties of M-estimation of linear regression with dependent errors, derived Bahadur representations of M-estimators and established a central limit theorem. We will obtain the central limit theorem for LSE and BLUE using some results of that paper.

Consider the linear model (3.0.1), we call the estimator minimizing

$$
\sum_{i=1}^{n} \rho\left(y_{t}-X_{t}^{\prime} \beta\right)
$$

an M-estimator, a name which stands for maximum-likelihood-type estimator (Huber (1981), page 43), where $\rho$ is a convex function. Note that the choice $\rho(x ; \theta)=-\log f(x ; \theta)$, where $f$ is the p.d.f. of the errors, corresponds to classical MLE for $\beta$. There are several important examples of M-estimators, such as Huber's estimator with

$$
\rho(x)=\left\{\begin{array}{ll}
\frac{1}{2} x^{2} & \text { if }|x| \leq \mathrm{k}, \\
k|x|-\frac{k^{2}}{2} & \text { if }|x|>\mathrm{k},
\end{array}, \mathrm{k}>0\right.
$$

The $\mathcal{L}^{q}$ regression estimator with 


$$
\rho(x)=|x|^{q}, 1 \leq q \leq 2,
$$

and the regression quantiles with

$$
\rho(x)=\rho_{\alpha}(x)=\alpha x^{+}+(1-\alpha)(-x)^{+}, 0<\alpha<1,
$$

where $x^{+}=\max (x, 0)$. The least absolute deviation (LAD) estimator is the value that minimizes (5.0.1), corresponding to $q=1(\rho(x)=|x|)$ or $\alpha=\frac{1}{2}\left(\rho(x)=\frac{1}{2}|x|\right)$ in the last two forms of $\rho$. In addition, the least square estimate is also a special case of the M-estimate with

$$
\rho(x)=x^{2}
$$

We are interested in the asymptotic behavior of M-estimation of linear regression with dependent errors. Suppose $\hat{\beta}_{n}$ minimizes $(5.0 .1)$ and $\beta_{0}$ is the true parameter. We establish a Bahadur representation that yields a consistent estimator of $\hat{\beta}_{n}$, and a central limit theorem for $\hat{\beta}_{n}-\beta_{0}$ for (3.0.1). The Bahadur representations are used to study the asymptotic behavior of an estimator by approximating it by a linear combination.

Suppose the errors $\left(\epsilon_{t}\right)$ form a stationary causal process in (3.0.1). By causal process, we mean that we can use a measurable function $G$ and some independent and identically distributed (i.i.d) random variables $e_{k}, k \in \mathbb{Z}$ ( $\mathbb{Z}$ is the integer set) to interpret the random variable $\epsilon_{t}$ like

$$
\epsilon_{i}=G\left(\ldots, e_{i-1}, e_{i}\right)
$$

This family contains the very popular class of linear processes (or infinite moving average process), 


$$
\epsilon_{i}=\sum_{j=0}^{\infty} a_{j} e_{i-j}
$$

which will be considered as an application for the rest of this chapter. Without loss of generality, let $a_{0}=1$.

Denote $\mathcal{F}_{k}=\left(\ldots, e_{k-1}, e_{k}\right)$. The conditional distribution function of $\epsilon_{1}$ at $u$ given $\mathcal{F}_{0}$ is

$$
F_{i}\left(u \mid \mathcal{F}_{0}\right)=P\left(\epsilon_{i} \leq u \mid \mathcal{F}_{0}\right)
$$

and the respective conditional density function is $f_{i}\left(u \mid \mathcal{F}_{0}\right)$. A function $g \in \mathcal{C}^{\prime}$ if $g$ has derivatives up to $l$ th-order and $g^{(l)}$ is continuous. The $l$ th-other derivative of the conditional density function is denoted by

$$
f_{i}^{(l)}\left(u \mid \mathcal{F}_{0}\right)=\frac{\partial^{\prime} f_{i}\left(u \mid \mathcal{F}_{0}\right)}{\partial u^{l}}
$$

if it exists. Let $e_{0}^{*}$ be an independent copy of $e_{0}, \mathcal{F}_{k}^{*}=\left(\ldots, e_{-1}, e_{0}^{*}, e_{1}, \ldots, e_{k}\right)$ and $\epsilon_{i}^{*}=G\left(\mathcal{F}_{k}^{*}\right)$. If $j<0, \mathcal{F}_{j}^{*}=\mathcal{F}_{j}$. Then $\epsilon_{i}^{*}$ and $\epsilon_{i}$ are identically distributed.

\subsection{Regularity Conditions}

As the M-estimators are regression equivariant, assume that without loss of generality $\beta_{0}=0$ in this chapter. The regression equivariant property of M-estimators follows from

$$
\sum_{i=1}^{n} \rho\left(y_{t}-X_{i}^{\prime} \beta\right)=\sum_{i=1}^{n} \rho\left(\left\{y_{i}+X_{i}^{\prime} v\right\}-X_{i}^{\prime}\{\beta+v\}\right)
$$


If we have $\hat{\beta}$ minimizing $\sum_{i=1}^{n} \rho\left(y_{1}-X_{i}^{\prime} \beta\right)$, we have

$$
\begin{aligned}
& \sum_{i=1}^{n} \rho\left(y_{t}-X_{i}^{\prime} \hat{\beta}\right) \leq \sum_{i=1}^{n} \rho\left(y_{t}-X_{i}^{\prime} \beta\right) \\
\Leftrightarrow & \sum_{i=1}^{n} \rho\left(\left\{y_{l}+X_{i}^{\prime} v\right\}-X_{i}^{\prime}\{\hat{\beta}+v\}\right) \leq \sum_{i=1}^{n} \rho\left(\left\{y_{i}+X_{i}^{\prime} v\right\}-X_{i}^{\prime}\{\beta+v\}\right) .
\end{aligned}
$$

Hence, we see that $\hat{\beta}+v$ minimizes $\sum_{i=1}^{n} \rho\left(\left\{y_{t}+X_{t}^{\prime} v\right\}-X_{t}^{\prime}\{\beta+v\}\right)$.

Let $X=\left(X_{1}, X_{2}, \cdots, X_{n}\right)^{\prime}$ and $\Sigma_{n}=X^{\prime} X$, which is nonsingular for large $\mathrm{n}$ by assumption. Rescale the model (3.0.1) into a more convenient form, such as

$$
y_{i}=Z_{i, n}^{\prime} \theta+\epsilon_{i},
$$

where $Z_{i, n}=\sum_{n}^{-1 / 2} X_{i}$ and $\theta=\sum_{n}^{1 / 2} \beta$. Hence the study of the asymptotic behavior of $\hat{\beta}_{n}$ is equivalent to that of $\hat{\theta}=\sum_{n}^{1 / 2} \hat{\beta}_{n} . \hat{\theta}$ is also obtained by the minimization of

$$
\sum_{i=1}^{n} \rho\left(\epsilon_{i}-Z_{i, n}^{\prime} \theta\right)=\sum_{i=1}^{n} \rho\left(y_{i}-Z_{i, n}^{\prime} \theta\right)
$$

as $\beta_{0}=0$ by assumption. We have

$$
\begin{aligned}
\sum_{i=1}^{n} Z_{i, n} Z_{i, n}^{\prime} & =\sum_{i=1}^{n} \sum_{n}^{-1 / 2} X_{i} X_{i}^{\prime}\left(\sum_{n}^{-1 / 2}\right)^{\prime}=\sum_{n}^{-1 / 2}\left(\sum_{i=1}^{n} X_{i} X_{i}^{\prime}\right) \sum_{n}^{-1 / 2} \\
& =\left(X^{\prime} X\right)^{-1 / 2} \sum_{i=1}^{n} X_{i} X_{i}^{\prime}\left(X^{\prime} X\right)^{-1 / 2} \\
& =\left(X^{\prime} X\right)^{-1 / 2}\left(X^{\prime} X\right)\left(X^{\prime} X\right)^{-1 / 2}=I_{p}
\end{aligned}
$$

the $p \times p$ identity matrix. We define

$$
\zeta_{n}=\sum_{i=1}^{n}\left|Z_{i, n}\right|^{q} \text { and } \xi_{n}=\sum_{i=1}^{n}\left|X_{i}\right|^{q}
$$

Suppose that $\rho^{\prime}=\psi \quad$ exists and define the $k$ th-step-ahead predicted function 


$$
\psi_{k}\left(t ; \mathcal{F}_{0}\right)=E\left[\psi\left(\epsilon_{k}+t\right) \mid \mathcal{F}_{0}\right], k \geq 0
$$

There are four regularity conditions on $\rho, X_{i}$ and the errors $\epsilon_{i}$ :

(A1) $\rho$ is a convex function, $E\left[\psi\left(\epsilon_{1}\right)\right]=0$ and $\left\|\psi\left(\epsilon_{1}\right)\right\|^{2}>0$;

There will be no limit on the variance of the error process $\left(\epsilon_{i}\right)$.

(A2) $\varphi(t)=E\left[\psi\left(\epsilon_{1}+t\right)\right]$ has a positive derivative at $t=0$;

Under this condition, $\theta$ is estimable (or separable): Let $F$ be a cumulative distribution function. If we have $F_{\theta}=F_{\theta^{\prime}}$, then $\theta=\theta^{\prime}$.

(A3) $m(t)=\left\|\psi\left(\epsilon_{1}+t\right)-\psi\left(\epsilon_{1}\right)\right\|$ is continuous at $t=0$;

This condition is not very restrictive and holds if the distribution of the error $\epsilon_{i}$ is continuous and for some $t_{0}>0\left\|\psi\left(\epsilon_{i}+t_{0}\right)\right\|+\left\|\psi\left(\epsilon_{i}-t_{0}\right)\right\|<\infty$. We know that $\psi$ is nondecreasing, and therefore it has countably many discontinuity points. We have $\psi\left(\epsilon_{i}(\omega)+t\right) \rightarrow \psi\left(\epsilon_{i}(\omega)\right)$ as $t \rightarrow 0$ for almost every $\omega$. For $0 \leq t \leq t_{0}$, we have

$$
\begin{aligned}
& \epsilon_{i}-t_{0} \leq \epsilon_{i}+t \leq \epsilon_{i}+t_{0} \\
& \Leftrightarrow-\left(\epsilon_{i}+t_{0}\right) \leq-\left(\epsilon_{i}+t\right) \leq-\left(\epsilon_{i}-t_{0}\right),
\end{aligned}
$$

so that $\left|\epsilon_{i}+t\right| \leq \max \left(\epsilon_{i}+t_{0},-\left(\epsilon_{i}-t_{0}\right)\right) \leq \max \left(\left|\epsilon_{i}+t_{0}\right|,\left|\epsilon_{i}-t_{0}\right|\right)$. Since $\psi$ is nondecreasing, we have if $0 \leq t \leq t_{0}$,

$$
\left\{\begin{array}{l}
\psi\left(\epsilon_{i}-t_{0}\right) \leq \psi\left(\epsilon_{i}+t\right) \leq \psi\left(\epsilon_{i}+t_{0}\right) \\
-\psi\left(\epsilon_{i}+t_{0}\right) \leq-\psi\left(\epsilon_{i}+t\right) \leq-\psi\left(\epsilon_{i}-t_{0}\right)
\end{array}\right.
$$


then

$$
\begin{aligned}
\left|\psi\left(\epsilon_{i}+t\right)\right| & \leq \max \left[\psi\left(\epsilon_{i}+t_{0}\right),-\psi\left(\epsilon_{i}-t_{0}\right)\right] \\
& \leq\left|\psi\left(\epsilon_{i}+t_{0}\right)\right|+\left|\psi\left(\epsilon_{i}-t_{0}\right)\right| ;
\end{aligned}
$$

and if $-t_{0} \leq t \leq 0$,

$$
\left\{\begin{array}{l}
\psi\left(\epsilon_{i}+t_{0}\right) \leq \psi\left(\epsilon_{i}+t\right) \leq \psi\left(\epsilon_{i}-t_{0}\right) \\
-\psi\left(\epsilon_{i}-t_{0}\right) \leq-\psi\left(\epsilon_{i}+t\right) \leq-\psi\left(\epsilon_{i}+t_{0}\right)
\end{array}\right.
$$

then

$$
\begin{aligned}
\left|\psi\left(\epsilon_{i}+t\right)\right| & \leq \max \left[\psi\left(\epsilon_{i}-t_{0}\right),-\psi\left(\epsilon_{i}+t_{0}\right)\right] \\
& \leq\left|\psi\left(\epsilon_{i}+t_{0}\right)\right|+\left|\psi\left(\epsilon_{i}-t_{0}\right)\right| .
\end{aligned}
$$

Hence we have

$$
\left|\psi\left(\epsilon_{i}+t\right)\right| \leq\left|\psi\left(\epsilon_{i}+t_{0}\right)\right|+\left|\psi\left(\epsilon_{i}-t_{0}\right)\right|, \quad \forall|t| \leq t_{0} .
$$

As

$$
\left\|\psi\left(\epsilon_{i}+t_{0}\right)\right\|+\left\|\psi\left(\epsilon_{i}-t_{0}\right)\right\|=\left(\int_{\Omega}\left|\psi\left(\epsilon_{i}+t_{0}\right)\right|^{2} d P\right)^{1 / 2}+\left(\int_{\Omega}\left|\psi\left(\epsilon_{i}-t_{0}\right)\right|^{2} d P\right)^{1 / 2}<\infty
$$

then $\left|\psi\left(\epsilon_{i}+t_{0}\right)\right|+\left|\psi\left(\epsilon_{i}-t_{0}\right)\right|=g$ is integrable as well. Hence we get

$$
E\left[\psi\left(\epsilon_{i}+t\right)\right] \rightarrow E\left[\psi\left(\epsilon_{i}\right)\right] \text { as } t \rightarrow 0,
$$

by the Lebesgue dominated convergence theorem.

From (5.1.4), we also have

$$
\left|\psi\left(\epsilon_{i}+t\right)-\psi\left(\epsilon_{i}\right)\right| \leq\left|\psi\left(\epsilon_{i}+t\right)\right|+\left|\psi\left(\epsilon_{i}\right)\right| \leq 2 g,
$$

where $2 g$ is integrable as well. Applying the Lebesgue dominated convergence theorem again, results in

$$
\left\|\psi\left(\epsilon_{i}+t\right)-\psi\left(\epsilon_{i}\right)\right\| \rightarrow E[0]=0 \text { as } t \rightarrow 0 .
$$


(A4) $r_{n}:=\max _{i \leq n}\left|Z_{i, n}\right|=\max _{i \leq n}\left(X_{i}^{\prime} \sum_{n}^{-1} X_{i}\right)^{\frac{1}{2}}=o(1)$.

With this condition, the diagonal elements of the matrix $X \sum_{n}^{-1} X^{\prime}$ are uniformly negligible in $i\left(\max _{i \leq n}\left(X_{i}^{\prime} \Sigma_{n}^{-1} X_{i}\right)^{\frac{1}{2}} \rightarrow 0\right)$, since $\left(X \Sigma_{n}^{-1} X^{\prime}\right)_{i i}=X_{i} \Sigma_{n}^{-1} X_{t}^{\prime} \rightarrow 0$ as $n \rightarrow \infty$. Let $1 \leq i_{1} \leq i_{2} \cdots \leq i_{p}$ and $X_{i_{1}}, \cdots, X_{i_{p}}$ be linear independent. Then $Q=\left(X_{i_{1}}, \cdots, X_{i_{p}}\right)$ is nonsingular. If $\sum_{n}$ is nonnegative definite, $\sum_{n}^{-1}$ is also nonnegative definite and so is $Q^{\prime} \Sigma_{n}^{-1} Q$. By the nonnegative definiteness of matrices, if the diagonal elements of $Q^{\prime} \sum_{n}^{-1} Q$ go to zero, $Q^{\prime} \sum_{n}^{-1} Q=A_{n} \rightarrow 0$. Hence we get $\Sigma_{n}^{-1}=\left(Q^{\prime}\right)^{-1} A_{n} Q^{-1} \rightarrow 0$. Consequently the eigenvalues of $\Sigma_{n}$ diverge to $\infty$ as $n \rightarrow \infty$.

\subsection{Bahadur Representation}

We introduce the theorem about the asymptotic normality of Bahadur representations of

M-estimators under certain conditions. In this theorem, we will approximate $\hat{\theta}_{n}$ by the linear form $T_{n}=\sum_{i=1}^{n} \psi\left(\epsilon_{i}\right) Z_{i, n}$.

THEOREM: Assume (A1)-(A4) and for some $t_{o}>0$,

$$
\sum_{i=1}^{\infty} \sup _{|l| \leq t_{0}}\left\|E\left[\psi\left(\epsilon_{i}+t\right) \mid \mathcal{F}_{0}\right]-E\left[\psi\left(\epsilon_{i}^{*}+t\right) \mid \mathcal{F}_{0}^{*}\right]\right\|<\infty
$$

Then we have 


$$
\varphi^{\prime}(0) \hat{\theta}_{n}-\sum_{i=1}^{n} \psi\left(\epsilon_{i}\right) Z_{i, n}=o_{\mathrm{P}}(1)
$$

and $\hat{\theta}_{n}=O_{p}(1)$. Additionally, if the limit

$$
\lim _{n \rightarrow \infty} \sum_{i=1}^{n-|k|} Z_{i, n} Z_{i+k, n}^{\prime}=\Delta_{k}
$$

exists for each $k \in \mathbb{Z}$, then

$$
\varphi^{\prime}(0) \hat{\theta}_{n} \Rightarrow N(0, \Delta), \quad \text { where } \Delta=\sum_{k \in \mathbb{Z}} E\left[\psi\left(\epsilon_{0}\right) \psi\left(\epsilon_{k}\right)\right] \Delta_{k} .
$$

As $\hat{\theta}=\sum_{n}^{\frac{1}{2}} \hat{\beta} \Rightarrow N\left(0,\left(\varphi^{\prime}(0)\right)^{-2} \Delta\right)$ and $\sum_{n}^{\frac{1}{2}} \rightarrow \infty$, we must have $\hat{\beta} \stackrel{P}{\longrightarrow} 0=\beta_{0}$. Therefore $\hat{\beta}$ is consistent.

Next, we will give a sufficient condition for (5.2.1). We define that $\psi\left(u ; t_{0}\right)=\left|\psi\left(u+t_{0}\right)\right|+\left|\psi\left(u-t_{0}\right)\right|$ and recall that the conditional distribution function of $\epsilon_{t}$ at $u$ given $\mathcal{F}_{0}$ is given by (5.0.4), and the respective conditional density function is $f_{i}\left(u \mid \mathcal{F}_{0}\right)$.

PROPOSITION 1: Condition (5.2.1) holds under

$$
\sum_{i=1}^{\infty} \bar{\omega}(i)<\infty, \text { where } \bar{\omega}(i)=\iint_{\bar{j}} \mid f_{i}\left(u \mid \mathcal{F}_{0}\right)-f_{i}\left(u \mid \mathcal{F}_{0}^{*}\right) \| \psi\left(u ; t_{0}\right) d u
$$

Proof of Proposition 1: This result can be seen as follows: using the fact that

$$
E\left[\psi\left(\epsilon_{i}+t\right) \mid \mathcal{F}_{0}\right]=\int_{\mathbb{E}} \psi(u+t) f_{\imath}\left(u \mid \mathcal{F}_{0}\right) d u
$$


and

$$
E\left[\psi\left(\epsilon_{i}^{*}+t\right) \mid \mathcal{F}_{0}^{*}\right]=\int_{\mathbb{i}_{*}^{*}} \psi(u+t) f_{i}\left(u \mid \mathcal{F}_{0}^{*}\right) d u
$$

We get for $|t| \leq t_{n}$,

$$
\begin{aligned}
\left\|E\left[\psi\left(\epsilon_{i}+t\right) \mid \mathcal{F}_{0}\right]-E\left[\psi\left(\epsilon_{i}^{*}+t\right) \mid \mathcal{F}_{0}^{*}\right]\right\| & =\left\|\int_{\mathbb{R}} \psi(u+t) f_{i}\left(u \mid \mathcal{F}_{0}\right) d u-\int_{\mathbb{E}} \psi(u+t) f_{i}\left(u \mid \mathcal{F}_{0}^{*}\right) d u\right\| \\
& \leq\left\|\int_{\mathbb{R}}\left|\psi(u+t) f_{i}\left(u \mid \mathcal{F}_{0}\right)-\psi(u+t) f_{i}\left(u \mid \mathcal{F}_{0}^{*}\right)\right| d u\right\| \\
& \leq \int\left\|_{\mathbb{R}}\left|f_{i}\left(u \mid \mathcal{F}_{0}\right)-f_{i}\left(u \mid \mathcal{F}_{0}^{*}\right) \|\right| \psi(u+t) \mid d u\right. \\
& \leq \int\left\|_{\mathbb{R}}\left|f_{i}\left(u \mid \mathcal{F}_{0}\right)-f_{i}\left(u \mid \mathcal{F}_{0}^{*}\right) \|\right| \psi\left(u ; t_{0}\right) \mid d u=\bar{\omega}(i)\right.
\end{aligned}
$$

and hence

$$
\sup _{|n| \leq t_{o}}\left\|E\left[\psi\left(\epsilon_{i}+t\right) \mid \mathcal{F}_{0}\right]-E\left[\psi\left(\epsilon_{i}^{*}+t\right) \mid \mathcal{F}_{0}^{*}\right]\right\| \leq \bar{\omega}(i)
$$

Interpretation of (5.2.5): If the conditional distribution $\left[\epsilon_{i} \mid \mathcal{F}_{0}\right]$ (of $\epsilon_{i}$ given $\mathcal{F}_{0}$ ) does not depend on $e_{0}$, then $f_{i}\left(u \mid \mathcal{F}_{0}\right)-f_{i}\left(u \mid \mathcal{F}_{0}^{*}\right)=0$. We use the function $\psi=\rho^{\prime}$ as weights in computing the distance between the two predictive distributions $\left[\epsilon_{i} \mid \mathcal{F}_{0}\right]$ and $\left[\epsilon_{i} \mid \mathcal{F}_{0}^{*}\right]$. Then the quantity $\bar{\omega}(i)=\int_{\mathbb{G}}\left\|f_{i}\left(u \mid \mathcal{F}_{0}\right)-f_{i}\left(u \mid \mathcal{F}_{0}^{*}\right)\right\| \psi\left(u ; t_{0}\right) d u$ can be interpreted as the cumulative contribution of $t_{0}$ in predicting $\epsilon_{i}$, which is finite. If the sum of $\bar{\omega}(i)$ is finite, we say that we have short range dependence.

There are two more conditions on the behavior of the function $\psi_{1}\left(s ; \mathcal{F}_{i}\right)$ at the neighborhood of $s=0$, that are necessary to obtain convergence rates for M-estimators 
of linear regression with dependent errors.

(A5) There exists an $t_{0}>0$ such that

$$
L_{i}:=\sup _{|s|,|| \mid \leq t_{0}, s \neq i} \frac{\left|\psi_{1}\left(s ; \mathcal{F}_{i}\right)-\psi_{1}\left(t ; \mathcal{F}_{i}\right)\right|}{|s-t|} \in \mathcal{L}^{\prime}
$$

Under this condition, the function $\psi_{1}\left(s ; \mathcal{F}_{i}\right),|s| \leq t_{0}$, is stochastically Lipschitz and hence continuous at a neighborhood of 0 , but the function $\psi$ itself does not have to be so.

(A6) Let $\psi_{1}\left(t ; \mathcal{F}_{i}\right) \in \mathbb{C}^{\prime}, l \geq 0, \sup _{|l| \leq t_{0}} \mid \psi_{1}^{(l)}\left(t ; \mathcal{F}_{i}\right) \|<\infty$ and

$$
\sum_{i=0}^{\infty} \sup _{|l| \leq t_{0}}\left\|E\left[\psi_{1}^{(\prime)}\left(t ; \mathcal{F}_{i}\right) \mid \mathcal{F}_{0}\right]-E\left[\psi_{1}^{(l)}\left(t ; \mathcal{F}_{i}^{*}\right) \mid \mathcal{F}_{0}^{*}\right]\right\|<\infty
$$

This is a generalization of condition (5.2.1) and also we are going to have a sufficient condition for (5.2.7) in Proposition 2.

PROPOSITION 2: Assume that $f_{1}\left(\cdot \mid \mathcal{F}_{i}\right) \in \mathcal{C}^{l}, l \geq 0$, and

$$
\sum_{i=1}^{\infty} \bar{\omega}_{l}(i)<\infty, \text { where } \bar{\omega}_{l}(i)=\iint_{\mathbb{E}}\left\|f_{1}^{(l)}\left(u \mid \mathcal{F}_{i}\right)-f_{1}^{(l)}\left(u \mid \mathcal{F}_{i}^{*}\right)\right\| \psi\left(u ; t_{0}\right) d u
$$

Then

$$
\sum_{i=0}^{\infty} \sup _{|l| t_{0}}\left\|\psi_{1}^{(l)}\left(t ; \mathcal{F}_{i}\right)-\psi_{1}^{(l)}\left(t ; \mathcal{F}_{i}^{*}\right)\right\|<\infty
$$

and (5.2.7) holds. 
Proof of Proposition 2: The proof of Proposition 2 will use the Lemma 1 given below, whose proof is given at the end. Recall the projection operators $\mathcal{P}_{k} \cdot=E\left(\cdot \mid \mathcal{F}_{k}\right)-E\left(\cdot \mid \mathcal{F}_{k-1}\right)$ and $\mathcal{F}_{k}^{*}=\left(\ldots, e_{-1}, e_{0}^{*}, e_{1}, \ldots, e_{k}\right)$.

LEMMA 1: Assume that the process $X_{t}=g\left(\mathcal{F}_{t}\right) \in \mathcal{L}^{2}$. Let $g_{n}\left(\mathcal{F}_{0}\right)=E\left[g\left(\mathcal{F}_{n}\right) \mid \mathcal{F}_{0}\right], n \geq 0 \quad$. Then $\quad\left\|g_{n}\left(\mathcal{F}_{0}\right)-g_{n}\left(\mathcal{F}_{0}^{*}\right)\right\| \leq\left\|g\left(\mathcal{F}_{n}\right)-g\left(\mathcal{F}_{n}^{*}\right)\right\| \quad$ and $\left\|\mathcal{P}_{0} X_{n}\right\| \leq\left\|g_{n}\left(\mathcal{F}_{0}\right)-g_{n}\left(\mathcal{F}_{0}^{*}\right)\right\| \leq 2\left\|\mathcal{P}_{0} X_{n}\right\| .(\mathrm{Wu}(2005))$

According to Lemma 1 and $\psi_{1}\left(t ; \mathcal{F}_{i}\right)=E\left[\psi\left(\epsilon_{t}+t\right) \mid \mathcal{F}_{i}\right]=\int_{i} \psi(v) f_{i}\left(v-t \mid \mathcal{F}_{i}\right) d v$, for $|t| \leq t_{0}$, we have

$$
\begin{aligned}
& \left\|E\left[\psi_{1}^{(l)}\left(t ; \mathcal{F}_{i}\right) \mid \mathcal{F}_{0}\right]-E\left[\psi_{1}^{(l)}\left(t ; \mathcal{F}_{i}^{*}\right) \mid \mathcal{F}_{0}^{*}\right]\right\| \\
& \leq\left\|\psi_{1}^{(l)}\left(t ; \mathcal{F}_{i}\right)-\psi_{1}^{(l)}\left(t ; \mathcal{F}_{i}^{*}\right)\right\| \\
& =\left\|\int_{\mathbb{R}^{*}} \psi(v) f_{i}\left(v-t \mid \mathcal{F}_{i}\right) d v-\int_{\mathbb{F}} \psi(v) f_{i}\left(v-t \mid \mathcal{F}_{i}^{*}\right) d v\right\| \\
& =\left\|\int_{\mathbb{R}} \psi(v)\left[f_{i}\left(v-t \mid \mathcal{F}_{i}\right)-f_{i}\left(v-t \mid \mathcal{F}_{i}^{*}\right)\right] d v\right\| \\
& \leq \int|\psi(v)|\left\|f_{i}\left(v-t \mid \mathcal{F}_{i}\right)-f_{i}\left(v-t \mid \mathcal{F}_{i}^{*}\right)\right\| d v .
\end{aligned}
$$

Let $u=v-t$. Since $\psi$ is nondecreasing, we have 


$$
\begin{aligned}
& \int_{\mathbb{E}}|\psi(v)|\left\|f_{i}\left(v-t \mid \mathcal{F}_{i}\right)-f_{i}\left(v-t \mid \mathcal{F}_{i}^{*}\right)\right\| d v \\
& =\int_{\mathbb{\pi}}|\psi(u+t)||| f_{i}\left(u \mid \mathcal{F}_{i}\right)-f_{i}\left(u \mid \mathcal{F}_{i}^{*}\right) \| d v \\
& \leq \int_{\bar{\tau}}\left(\left|\psi\left(u+t_{0}\right)\right|+\left|\psi\left(u-t_{0}\right)\right|\right)|| f_{i}\left(u \mid \mathcal{F}_{i}\right)-f_{i}\left(u \mid \mathcal{F}_{i}^{*}\right) \| d v \quad \forall|t| \leq t_{0} \\
& =\int_{\bar{\pi}} \psi\left(u ; t_{0}\right)\left\|f_{i}\left(u \mid \mathcal{F}_{i}\right)-f_{i}\left(u \mid \mathcal{F}_{i}^{*}\right)\right\| d v=\bar{\omega}_{l}(i)
\end{aligned}
$$

Therefore, (5.2.8) holds.

Proof of Lemma 1: (i ) $\left\|g_{n}\left(\mathcal{F}_{0}\right)-g_{n}\left(\mathcal{F}_{0}^{*}\right)\right\| \leq\left\|g\left(\mathcal{F}_{n}\right)-g\left(\mathcal{F}_{n}^{*}\right)\right\|$

Since $\mathcal{F}_{n}^{*}=\left(\ldots, e_{-1}, e_{0}^{*}, e_{1}, \ldots, e_{n}\right)$,

$$
\begin{aligned}
& E\left[g\left(\mathcal{F}_{n}\right)-g\left(\mathcal{F}_{n}^{*}\right) \mid \mathcal{F}_{-1}, e_{0}^{*}, e_{0}\right] \\
& =E\left[g\left(\mathcal{F}_{n}\right) \mid \mathcal{F}_{-1}, e_{0}\right]-E\left[g\left(\mathcal{F}_{n}^{*}\right) \mid \mathcal{F}_{-1}, e_{0}^{*}\right] \\
& =E\left[g\left(\mathcal{F}_{n}\right) \mid \mathcal{F}_{0}\right]-E\left[g\left(\mathcal{F}_{n}^{*}\right) \mid \mathcal{F}_{0}^{*}\right] \\
& =g_{n}\left(\mathcal{F}_{0}\right)-g_{n}\left(\mathcal{F}_{0}^{*}\right),
\end{aligned}
$$

which by Jensen's Inequality implies

$$
\begin{aligned}
& \left\|g_{n}\left(\mathcal{F}_{0}\right)-g_{n}\left(\mathcal{F}_{0}^{*}\right)\right\| \\
& =\left\|E\left[g\left(\mathcal{F}_{n}\right)-g\left(\mathcal{F}_{n}^{*}\right) \mid \mathcal{F}_{-1}, e_{0}^{*}, e_{0}\right]\right\| \\
& \leq E\left[\left\|g\left(\mathcal{F}_{n}\right)-g\left(\mathcal{F}_{n}^{*}\right)\right\| \mathcal{F}_{-1}, e_{0}^{*}, e_{0}\right] \\
& =\left\|g\left(\mathcal{F}_{n}\right)-g\left(\mathcal{F}_{n}^{*}\right)\right\| .
\end{aligned}
$$

(ii) $\left\|\mathcal{P}_{0} X_{n}\right\| \leq\left\|g_{n}\left(\mathcal{F}_{0}\right)-g_{n}\left(\mathcal{F}_{0}^{*}\right)\right\| \leq 2\left\|\mathcal{P}_{0} X_{n}\right\|$

We have $E\left[g_{n}\left(\mathcal{F}_{0}\right) \mid \mathcal{F}_{-1}\right]=E\left[E\left[g\left(\mathcal{F}_{n}\right) \mid \mathcal{F}_{0}\right] \mid \mathcal{F}_{-1}\right]=E\left[g\left(\mathcal{F}_{n}\right) \mid \mathcal{F}_{-1}\right]$ and 


$$
\begin{aligned}
E\left[g_{n}\left(\mathcal{F}_{0}^{*}\right) \mid \mathcal{F}_{0}\right] & =E\left[E\left[g\left(\mathcal{F}_{n}\right) \mid \mathcal{F}_{0}^{*}\right] \mid \mathcal{F}_{0}\right] \\
& =E\left[E\left[g\left(\mathcal{F}_{n}\right) \mid \mathcal{F}_{-1}\right] \mid \mathcal{F}_{0}\right]\left(e_{0}^{*} \text { is independent of } \mathcal{F}_{n}\right) \\
& =E\left[g\left(\mathcal{F}_{n}\right) \mid \mathcal{F}_{-1}\right] \\
& =E\left[E\left[g\left(\mathcal{F}_{n}\right) \mid \mathcal{F}_{0}\right] \mid \mathcal{F}_{-1}\right] \\
& =E\left[g_{n}\left(\mathcal{F}_{0}\right) \mid \mathcal{F}_{-1}\right]
\end{aligned}
$$

Then

$$
\begin{aligned}
\left\|\mathcal{P}_{0} X_{n}\right\| & =\left\|E\left[g\left(\mathcal{F}_{n}\right) \mid \mathcal{F}_{0}\right]-E\left[g\left(\mathcal{F}_{n}\right) \mid \mathcal{F}_{-1}\right]\right\| \\
& =\left\|g_{n}\left(\mathcal{F}_{0}\right)-E\left[g_{n}\left(\mathcal{F}_{0}^{*}\right) \mid \mathcal{F}_{0}\right]\right\| \\
& =\left\|E\left[g_{n}\left(\mathcal{F}_{0}\right) \mid \mathcal{F}_{0}\right]-E\left[g_{n}\left(\mathcal{F}_{0}^{*}\right) \mid \mathcal{F}_{0}\right]\right\| \\
& =\left\|E\left[g_{n}\left(\mathcal{F}_{0}\right)-g_{n}\left(\mathcal{F}_{0}^{*}\right) \mid \mathcal{F}_{0}\right]\right\| \\
& \leq\left\|g_{n}\left(\mathcal{F}_{0}\right)-g_{n}\left(\mathcal{F}_{0}^{*}\right)\right\| \\
& =\left\|g_{n}\left(\mathcal{F}_{0}\right)-E\left[g_{n}\left(\mathcal{F}_{0}\right) \mid \mathcal{F}_{-1}\right]+E\left[g_{n}\left(\mathcal{F}_{0}\right) \mid \mathcal{F}_{-1}\right]-g_{n}\left(\mathcal{F}_{0}^{*}\right)\right\| \\
& \leq\left\|g_{n}\left(\mathcal{F}_{0}\right)-E\left[g_{n}\left(\mathcal{F}_{0}\right) \mid \mathcal{F}_{-1}\right]\right\|+\left\|E\left[g_{n}\left(\mathcal{F}_{0}\right) \mid \mathcal{F}_{-1}\right]-g_{n}\left(\mathcal{F}_{0}^{*}\right)\right\| \\
& =2\left\|\mathcal{P}_{0} X_{n}\right\|
\end{aligned}
$$

since $g_{n}\left(\mathcal{F}_{0}\right)-E\left[g_{n}\left(\mathcal{F}_{0}\right) \mid \mathcal{F}_{-1}\right]$ and $E\left[g_{n}\left(\mathcal{F}_{0}\right) \mid \mathcal{F}_{-1}\right]-g_{n}\left(\mathcal{F}_{0}^{*}\right)$ have same distribution.

\subsection{Application to Infinite Moving Average Errors}

Here we will give a simpler sufficient condition than in Proposition 2 . Let $F_{e}$ be the distribution function of $e_{0}$ and $f_{e}$ be the density function of $e_{0}$. Also, let the weighted measure $w_{\gamma}(d u)=(1+|u|)^{\gamma} d u, \gamma \in \mathbb{R}$.

PROPOSITION 3: Let $1<\gamma<q$. Assume $E\left(e_{0}\right)=0, \quad e_{0} \in \mathcal{L}^{q}$, 
$k_{\gamma}=\int_{\vec{x}} \psi^{2}(u) \times w_{-\gamma}(d u)<\infty$ and

$$
\sum_{k=0}^{p+1} \iint_{\mathbb{R}} f_{e}^{(k)}(v) \mid w_{\gamma}(d v)<\infty
$$

Then $\bar{\omega}_{l}(i)=O\left(\left|a_{i}\right|^{q^{\prime / 2}}\right), 0 \leq l \leq p$, where $q^{\prime}=\min (2, q)$, and therefore (5.2.8) holds under

$$
\sum_{j=0}^{\infty}\left|a_{j}\right|^{q^{1 / 2}}<\infty
$$

This proposition will allow dependent and heavy-tailed errors, since $q^{1 / 2}=\min (1, q / 2) \leq 1$ which means the errors do not need to have a finite second moment. If we apply least square procedure to estimate the unknown $\beta$, this will result in estimators that are very sensitive to small changes in the values of the design $X_{t}$ or the errors $\epsilon$. Hence, it may be more appropriate to apply the M-estimation technique here. Since the moving average process is a popular model for heavy-tailed processes, we apply it in this study. The proof of Proposition 3 is from Wu (2006).

Proof of Proposition 3: We will do the proof for $k=0$. With the variable change $t=u-e$, 


$$
\begin{aligned}
\sup _{|e| \leq t_{0}} \int_{\mathbb{K}} \frac{\psi^{2}(t+e)}{(1+|t|)^{\gamma}} d t & =\sup _{|e| \leq t_{0}} \int_{\mathbb{E}} \frac{\psi^{2}(u)}{(1+|u-e|)^{\gamma}} d u \\
& \leq \sup _{|e| I_{0}} \int_{\mathbb{R}} \frac{\psi^{2}(t)}{(1+|u|)^{\gamma}}(1+|e|)^{\gamma} d u \\
& =\left(1+\left|t_{0}\right|\right)^{\gamma} k_{y}<\infty .
\end{aligned}
$$

The inequality above is true, because

$$
\begin{aligned}
& |u| \Rightarrow|u-e+e| \leq|u-e|+|e| \\
& \Leftrightarrow 1+|u| \leq 1+|u-e|+|e|+|u-e| \cdot|e| \\
& \Leftrightarrow 1+|u| \leq(1+|u-e|)(1+|e|) \\
& \Leftrightarrow \frac{1}{1+|u-e|} \leq \frac{1+|e|}{1+|u|} .
\end{aligned}
$$

Then from (5.2.11), we observe that $\int_{\mathbb{E}} \psi^{2}\left(t ; t_{0}\right) w_{-\gamma}(d t)<\infty$. Let $Z_{n}=\sum_{j=1}^{\infty} a_{j} e_{n-j}$,

$Z_{n}^{*}=Z_{n}-a_{n} e_{0}+a_{n} e_{0}^{*}$, and $U_{n}=Z_{n}-a_{n} e_{0}=Z_{n}^{*}-a_{n} e_{0}^{*}$. Also let

$$
R_{n}=\int\left[f_{\mathbb{R}}\left(t-U_{n}\right)-f_{e}\left(t-U_{n}-a_{n} e_{0}\right)\right]^{2} w_{r}(d t) .
$$

By Schwarz's inequality,

$$
\begin{aligned}
\bar{\omega}_{0}(n-1)^{2} & =\left(\iint_{\mathbb{E}}\left\|f_{1}\left(u \mid \mathcal{F}_{n-1}\right)-f_{1}\left(u \mid \mathcal{F}_{n-1}^{*}\right)\right\| \psi\left(u ; t_{0}\right) d u\right)^{2} \\
& \leq \int_{\mathbb{i}}\left\|f_{1}\left(u \mid \mathcal{F}_{n-1}\right)-f_{1}\left(u \mid \mathcal{F}_{n-1}^{*}\right)\right\|^{2} w_{\gamma}(d u) \cdot \int_{\bar{F}} \psi^{2}\left(u ; t_{0}\right) w_{-\gamma}(d u),
\end{aligned}
$$

where $F_{1}\left(u \mid \mathcal{F}_{n-1}\right)=\operatorname{Pr}\left(\epsilon_{n} \leq u \mid \mathcal{F}_{n-1}\right)$.

As we know that

$$
\begin{aligned}
F_{1}\left(u \mid \mathcal{F}_{n-1}\right) & =\operatorname{Pr}\left(\epsilon_{n} \leq u \mid \mathcal{F}_{n-1}\right) \\
& =\operatorname{Pr}\left(Z_{n}+e_{n} \leq u \mid \mathcal{F}_{n-1}\right) \\
& =\operatorname{Pr}\left(e_{n} \leq u-Z_{n} \mid \mathcal{F}_{n-1}\right) \\
& =\operatorname{Pr}\left(e_{n} \leq u-Z_{n}\right)=F_{e}\left(u-Z_{n}\right)
\end{aligned}
$$

we have 


$$
\begin{aligned}
\bar{\omega}_{0}(n-1)^{2} & =\int_{\bar{x}^{*}} \psi^{2}\left(u ; t_{0}\right) w_{-\gamma}(d u) \cdot \int_{\mathbb{E}} f_{e}\left(u-Z_{n}\right)-f_{e}\left(u-Z_{n}^{*}\right) \|^{2} w_{\gamma}(d u) \\
& \leq C \cdot \int\left[\left(\left\|f_{e}\left(u-Z_{n}\right)-f_{e}\left(u-Z_{n}^{*}+a_{n} e_{0}^{*}\right)\right\|\right)+\left(\left\|f_{e}\left(u-Z_{n}^{*}+a_{n} e_{0}^{*}\right)-f_{e}\left(u-Z_{n}^{*}\right)\right\|\right)\right]^{2} w_{y}(d u) \\
& =C \cdot \int\left(2 \cdot\left\|f_{e}\left(u-Z_{n}\right)-f_{e}\left(u-Z_{n}^{*}+a_{n} e_{0}^{*}\right)\right\|\right)^{2} w_{\gamma}(d u) \\
& =C \cdot \int_{\mathbb{R}} E\left[f_{e}\left(u-U_{n}-a_{n} e_{0}\right)-f_{e}\left(u-U_{n}\right)\right]^{2} w_{\gamma}(d u) \\
& =C \cdot E \int\left[f_{e}\left(u-U_{n}-a_{n} e_{0}\right)-f_{e}\left(u-U_{n}\right)\right]^{2} w_{\gamma}(d u) \\
& =C \cdot E\left(R_{n}\right),
\end{aligned}
$$

ere $\mathrm{C}$ is a constant that may change from line to line. Observe that

$$
\int_{\mathbb{E}} f_{e}^{2}(t-u) w_{\gamma}(d t) \leq(1+|u|)^{\gamma} \int_{\mathbb{E}} f_{e}^{2}(t) w_{\gamma}(d t)=I_{0}(1+|u|)^{\gamma},
$$

where $I_{k}=\int_{\bar{\varepsilon}}\left|f_{e}^{(k)}(t)\right|^{2} w_{y}(d t)$, since

$$
\begin{aligned}
& |s+u| \leq|s|+|u| \\
& \Rightarrow 1+|s+u| \leq 1+|s|+|u|+|s| \cdot|u| \\
& \Rightarrow 1+|s+u| \leq(1+|s|)(1+|u|) \\
& \Rightarrow \int_{\mathbb{R}} f_{e}^{2}(s)(1+|s+u|)^{\gamma} d t \leq \int_{\mathbb{R}} f_{e}^{2}(s)(1+|s|)^{\gamma}(1+|u|)^{\gamma} d t .
\end{aligned}
$$

By substituting $s=t-u$ and $t=s+u$ into the above inequality, we get the inequality we are seeking. Then

$$
\begin{aligned}
R_{n} & =\int_{\mathbb{x}}\left[f_{e}^{2}\left(t-U_{n}\right)+f_{e}^{2}\left(t-U_{n}-a_{n} e_{0}\right)-2 f_{e}^{2}\left(t-U_{n}\right) f_{e}^{2}\left(t-U_{n}-a_{n} e_{0}\right)\right] w_{y}(d t) \\
& \leq \int_{j} f_{e}^{2}\left(t-U_{n}\right) w_{\gamma}(d t)+\int_{\mathbb{R}} f_{e}^{2}\left(t-U_{n}-a_{n} e_{0}\right) w_{\gamma}(d t) \\
& \leq C \cdot\left[\left(1+\left|U_{n}\right|\right)^{\gamma}+\left(1+\left|U_{n}+a_{n} e_{0}\right|\right)^{\gamma}\right] \\
& \leq C \cdot\left[\left(1+\left|U_{n}\right|\right)^{y}+\left|a_{n} e_{0}\right|^{\gamma}\right] .
\end{aligned}
$$

On the other hand, since 


$$
\begin{aligned}
\int_{v}\left|\int_{0}^{a_{n} e_{0}}\left[f_{e}\left(t-U_{n}-v\right)\right]^{2} d v\right| w_{\gamma}(d t) & \leq\left|\int_{0}^{a_{n} e_{0}}\left[\int_{d=0}^{\prime}\left(t-U_{n}-v\right)\right]^{2} w_{\gamma}(d t) d v\right| \\
& \leq I_{1}\left|\int_{0}^{a_{n} e_{0}}\left(1+\left|U_{n}+v\right|\right)^{\gamma} d v\right| \\
& \leq C \cdot\left|a_{n} e_{0}\right|\left[\left(1+\left|U_{n}\right|\right)^{\gamma}+\left|a_{n} e_{0}\right|^{\gamma}\right]
\end{aligned}
$$

and $\int_{0}^{s} f_{e}^{\prime}(t-u-v) d v=f_{e}(t-u)-f_{e}(t-u-s)$, we have

$$
\begin{aligned}
& R_{n}=\int_{\mathbb{j}}\left[f_{e}\left(t-U_{n}\right)-f_{e}\left(t-U_{n}-a_{n} e_{0}\right)\right]^{2} w_{\gamma}(d t) \\
& =\int\left(\int_{e}^{a_{n} e_{0}}\left[f_{e}^{\prime}\left(t-U_{n}-v\right)\right] d v\right)^{2} w_{y}(d t) \\
& \left.\leq \int_{(}^{a_{n} e_{0}}\left[\int_{0}^{\prime}\left[t-U_{n}-v\right)\right]^{2} d v \cdot \int_{0}^{a_{n} e_{0}} 1^{2} d v\right) w_{y}(d t) \\
& =\left|a_{n} e_{0}\right| \iint_{i \mathrm{k}} \int_{0}^{a_{n} e_{0}}\left[f_{e}^{\prime}\left(t-U_{n}-v\right)\right]^{2} d v \mid w_{\gamma}(d t) \\
& \leq C \cdot\left|a_{n} e_{0}\right|^{2}\left[\left(1+\left|U_{n}\right|\right)^{\gamma}+\left|a_{n} e_{0}\right|^{\gamma}\right] \text {. }
\end{aligned}
$$

To summarize, we have

$$
R_{n} \leq \min \left\{C \cdot\left[\left(1+\left|U_{n}\right|\right)^{y}+\left|a_{n} e_{0}\right|^{\gamma}\right], C \cdot\left|a_{n} e_{0}\right|^{2}\left[\left(1+\left|U_{n}\right|\right)^{y}+\left|a_{n} e_{0}\right|^{\gamma}\right]\right\} \cdot
$$

Since $a_{n} e_{0}$ and $U_{n}$ are independent and $E\left(\left|U_{n}\right|^{q}\right)<\infty$ and $1<\gamma<q$, by above inequality,

$$
\begin{aligned}
E\left(R_{n}\right) & \leq C \cdot E\left[\min \left(\left|a_{n} e_{0}\right|^{2}, 1\right) \cdot\left(1+\left|U_{n}\right|\right)^{\gamma}\right]+C \cdot E\left[\min \left(\left|a_{n} e_{0}\right|^{2}, 1\right) \cdot\left|a_{n} e_{0}\right|^{\gamma}\right] \\
& \leq C \cdot E\left[\left|a_{n} e_{0}\right|^{q^{\prime}} \cdot\left(1+\left|U_{n}\right|\right)^{\gamma}\right]+C \cdot E\left[\left|a_{n} e_{0}\right|^{\min (2, q-\gamma)} \cdot\left|a_{n} e_{0}\right|^{\gamma}\right] \\
& =O\left(\left|a_{n}\right|^{q^{\prime}}\right) .
\end{aligned}
$$

The general case with $k \geq 1$ follows similarly. 
The following lemma is useful to study the asymptotic behavior of M-estimators.

LEMMA 2: Let $T_{n}=\sum_{i=1}^{n} \psi\left(\epsilon_{i}\right) Z_{i, n}$.

(i) Assume $E\left[\psi\left(\epsilon_{i}\right)\right]=0,\left\|\psi\left(\epsilon_{i}\right)\right\|<\infty$ and that (5.2.10) holds,

$$
\sum_{i=1}^{\infty}\left\|E\left[\psi\left(\epsilon_{i}\right) \mid \mathcal{F}_{0}\right]-E\left[\psi\left(\epsilon_{i}^{*}\right) \mid \mathcal{F}_{0}^{*}\right]\right\|<\infty
$$

Then $\left\|T_{n}\right\|=O(1)$.

(ii) If in addition (5.2.3) and (A4) hold, then $T_{n} \Rightarrow N(0, \Delta)$.

\subsection{Application to Linear processes}

First, we are going to apply Lemma 2 to establish a central limit theorem for Least square estimator when errors come from a linear process. The errors follows a moving average process

$$
\epsilon_{i}=\sum_{j=0}^{\infty} a_{j} e_{i-j} .
$$

Recall that for LSE, $\rho\left(\epsilon_{i}\right)=\epsilon_{i}^{2}$ and $\psi\left(\epsilon_{i}\right)=2 \epsilon_{i}$. Then we have $E\left[\psi\left(\epsilon_{i}\right)\right]=E\left[2 \epsilon_{i}\right]=0,\left\|\psi\left(\epsilon_{i}\right)\right\|=\left\|2 \epsilon_{i}\right\|<\infty$ and 


$$
\begin{aligned}
& \sum_{i=1}^{\infty}\left\|E\left[\psi\left(\epsilon_{i}\right) \mid \mathcal{F}_{0}\right]-E\left[\psi\left(\epsilon_{i}^{*}\right) \mid \mathcal{F}_{0}^{*}\right]\right\| \\
& =\sum_{i=1}^{\infty}\left\|E\left[2 \epsilon_{i} \mid \mathcal{F}_{0}\right]-E\left[2 \epsilon_{i}^{*} \mid \mathcal{F}_{0}^{*}\right]\right\| \\
& =2 \sum_{i=1}^{\infty}\left\|E\left[\sum_{j=0}^{i-1} a_{j} e_{i-j}+a_{i} e_{0}+\sum_{j=i+1}^{\infty} a_{j} e_{i-j} \mid \mathcal{F}_{0}\right]-E\left[\sum_{j=0}^{i-1} a_{j} e_{i-j}+a_{i} e_{0}^{*}+\sum_{j=i+1}^{\infty} a_{j} e_{i-j} \mid \mathcal{F}_{0}^{*}\right]\right\| \\
& =2 \sum_{i=1}^{\infty}\left\|a_{i} e_{0}-a_{i} e_{0}^{*}\right\|=2 \sum_{i=1}^{\infty}\left(E\left|a_{i} e_{0}-a_{i} e_{0}^{*}\right|^{2}\right)^{1 / 2}=2 \sum_{i=1}^{\infty}\left|a_{i}\right| E\left|e_{0}-e_{0}^{*}\right|^{2} \\
& =2 \sum_{i=1}^{\infty}\left|a_{i}\right|\left(E\left|e_{0}\right|^{2}+E\left|e_{0}^{*}\right|^{2}\right)=C \cdot \sum_{i=1}^{\infty}\left|a_{i}\right|^{2 \infty} .
\end{aligned}
$$

The conditions (5.2.3) and (A4) are on the design matrices. Hence if the design matrix satisfies (A4), we get $T_{n}=\sum_{i=1}^{n} 2 \epsilon_{i} \sum_{n}^{-\frac{1}{2}} X_{i} \Rightarrow N\left(0, \Delta_{L S E}\right)$, where

$$
\begin{aligned}
\Delta_{L S E} & =\sum_{k \in \mathbb{Z}}\left\{E\left[\psi\left(\epsilon_{0}\right) \psi\left(\epsilon_{k}\right)\right] \cdot \lim _{n \rightarrow \infty} \sum_{i=1}^{n-|k|} Z_{i, n} Z_{i+k, n}^{\prime}\right\} \\
& =\sum_{k \in \mathbb{Z}}\left\{E\left[2 \epsilon_{0} \cdot 2 \epsilon_{k}\right] \cdot \lim _{n \rightarrow \infty} \sum_{i=1}^{n-|k|} \sum_{n}^{-\frac{1}{2}} X_{i}\left(\sum_{n}^{-\frac{1}{2}} X_{i+k}\right)^{\prime}\right\} \\
& =4 \sum_{k \in \mathbb{Z}}\left\{E\left[\epsilon_{0} \epsilon_{k}\right] \cdot \lim _{n \rightarrow \infty} \sum_{n}^{-\frac{1}{2}}\left(\sum_{i=1}^{n-|k|} X_{i} X_{i+k}^{\prime}\right) \sum_{n}^{-\frac{1}{2}}\right\} .
\end{aligned}
$$

We can easily find that $\quad \hat{\beta}=\sum_{i=1}^{n} \epsilon_{i} X_{i} \Rightarrow N\left(0, \sum_{k \in \mathbb{Z}}\left\{E\left[\epsilon_{0} \epsilon_{k}\right] \cdot \lim _{n \rightarrow \infty}\left(\sum_{i=1}^{n-|k|} X_{i} X_{i+k}^{\prime}\right)\right\}\right) \quad$ from Theorem 1 and the above results.

For Best Linear Unbiased Estimator we can use the model in (3.1.1),

$$
z_{t}=B_{t}^{\prime} \beta+g_{\imath}
$$

We know that $R$ is the covariance matrix of $\epsilon$ and $K^{\prime} K=K K=R$. Also we have $z=K^{-1} y, B=K^{-1} X$ and $g=K^{-1} \epsilon . B_{t}=\left(B_{t 1}, B_{12}, \cdots, B_{t k}\right)^{\prime}$ regression variables, and 
$g=\left\{g_{1}\right\}_{t=1}^{n}$ is the vector of the new errors which has zero-mean and is uncorrelated.

For BLUE, we have $\rho\left(g_{i}\right)=g_{i}^{2}$ and $\psi\left(g_{i}\right)=2 g_{i}=2\left(K^{-1} \epsilon\right)_{i}$. Here we assume that $g=\left\{g_{l}\right\}_{t=1}^{n}$ follows an normal distribution with zero-mean (note that it would be better to adapt the procedure for non-normal case for future study). Then we have $E\left[\psi\left(g_{i}\right)\right]=0$, $\|\psi(g)\|_{i}=\|2 g\|_{i}<\infty$ and $\sum_{i=1}^{\infty}\left\|E\left[\psi\left(g_{i}\right) \mid \mathcal{F}_{0}\right]-E\left[\psi\left(g_{i}^{*}\right) \mid \mathcal{F}_{0}^{*}\right]\right\|<\infty$

Here $\Gamma_{n}=B_{n}^{\prime} B_{n}=\left(K^{-1} X\right)^{\prime}\left(K^{-1} X\right)=X^{\prime} R^{-1} X$. Conditions (5.2.3) and (A4) hold, so we have, where

$$
\begin{aligned}
\Delta_{B L U E} & =\sum_{k \in \mathbb{Z}} E\left[\psi\left(g_{0}\right) \psi\left(g_{k}\right)\right] \cdot \lim _{n \rightarrow \infty} \sum_{i=1}^{n-k \mid} \tilde{Z}_{i, n} \tilde{Z}_{i+k, n}^{\prime} \quad E\left[g_{0} g_{k}\right]=0, \text { if } k \neq 0 \\
& =4 E\left[g_{0}^{2}\right] \lim _{n \rightarrow \infty} \sum_{i=1}^{n}\left(\Gamma_{n}^{-1 / 2} B_{i}\right)\left(\Gamma_{n}^{-1 / 2} B_{i}\right)^{\prime} \\
& =4 E\left[g_{0}^{2}\right] \lim _{n \rightarrow \infty} \sum_{i=1}^{n}\left(B_{n}^{\prime} B_{n}\right)^{-1 / 2} B_{i} B_{i}^{\prime}\left(B_{n}^{\prime} B_{n}\right)^{-1 / 2} \\
& =4 E\left[g_{0}^{2}\right] \lim _{n \rightarrow \infty}\left(B_{n}^{\prime} B_{n}\right)^{-1 / 2}\left(\sum_{i=1}^{n} B_{i} B_{i}^{\prime}\right)\left(B_{n}^{\prime} B_{n}\right)^{-1 / 2} \\
& =4 E\left[g_{0}^{2}\right] \lim _{n \rightarrow \infty}\left(B_{n}^{\prime} B_{n}\right)^{-1 / 2} B_{n}^{\prime} B_{n}\left(B_{n}^{\prime} B_{n}\right)^{-1 / 2} \\
& =4 E\left[g_{0}^{2}\right] I_{p}=4 I_{p}
\end{aligned}
$$

Hence we get $\tilde{\beta}=\sum_{i=1}^{n} g_{i} B_{i}$ can be considered for large $\mathrm{n}$ as $N\left(0,\left(X^{\prime} R^{-1} X\right)^{-1}\right)$ from Theorem 1 and the above results. Also, $E\left[g_{0}^{2}\right]=1$. 


\section{Computer Simulation}

\subsection{Introduction}

We investigated the asymptotic efficiency of the LSE relative to the BLUE in linear regression with dependent errors in chapter 4 , and the central limit theorem for LSE and BLUE in chapter 5. Theoretically, we know that LSE is asymptotic efficient relative to BLUE, if and only if the spectral density of the errors is constant on each of the elements of $S$ (the regression spectrum). In addition, both LSE and BLUE follow the central limit theorem (chapter 5).

In this chapter, we illustrate the above conclusions based on the simulated data. In section 6.1, we will compare the covariance matrices of LSE and BLUE to see if the difference between them is small enough to be ignored. Next, in section 6.2 , we will generate data coming from a certain distribution, say a $\mathrm{t}$ distribution or a normal distribution, in order to simulate the Bahadur representations for LSE and BLUE $n$ times, say $n=500$. We can then use the normality test to determine if the estimator follows a normal distribution. 


\subsection{Simulation for Asymptotic Efficiency}

This section compares asymptotic covariance matrices of LSE and BLUE. First, we construct a design matrix, $\mathrm{X}$, which is either Polynomial regression or trigonometric regression. As we mentioned before, a trigonometric regression is of the form

$$
X_{t}^{(v)}=a \cos \left(\lambda_{v} t\right)
$$

and a polynomial regression of the form

$$
X_{t}^{(v)}=t^{v}
$$

According to the conclusion we made in this part, we need to design a covariance matrix,

$R$. Suppose the spectral density is

$$
f(\lambda)=\left\{\begin{array}{l}
a \text { if } \pi>|\lambda|>k \\
b \text { if } 0<|\lambda|<k
\end{array}, \text { where } k \in(0, \pi)\right.
$$

Then if $h=0$,

$$
r(0)=\int_{-\pi}^{\pi} e^{0} f(\lambda) d \lambda=\int_{-\pi}^{\pi} f(\lambda) d \lambda=a\left(\int_{k}^{\pi} d \lambda+\int_{-\pi}^{-k} d \lambda\right)+b \int_{-k}^{k} d \lambda=2 a(\pi-k)+2 b k .
$$

And if $h \neq 0$,

$$
\begin{aligned}
r(h) & =\int_{-\pi}^{\pi} e^{i h \lambda} f(\lambda) d \lambda=a\left(\int_{k}^{\pi} e^{i h \lambda} d \lambda+\int_{-\pi}^{-k} e^{i h \lambda} d \lambda\right)+b \int_{-k}^{k} e^{i h \lambda} f(\lambda) d \lambda \\
& =\frac{a}{h}\left(\left.[\sin (h \lambda)-i \cos (h \lambda)]\right|_{-\pi} ^{-k}+\left.[\sin (h \lambda)-i \cos (h \lambda)]\right|_{k} ^{\pi}\right)+\left.\frac{b}{h}[\sin (h \lambda)-i \cos (h \lambda)]\right|_{-k} ^{k} \\
& =\frac{a}{h}\{([\sin (-h k)-i \cos (-h k)]-[\sin (-h \pi)-i \cos (-h \pi)]) \\
& -([\sin (h k)-i \cos (h k)]-[\sin (h \pi)-i \cos (h \pi)])\} \\
& +\frac{b}{h}[([\sin (h k)-i \cos (h k)]-[\sin (-h k)-i \cos (-h k)])] \\
& =\frac{a}{h}(-2 \sin (h k))+\frac{b}{h}(2 \sin (h k))=2 \frac{b-a}{h} \sin (h k) .
\end{aligned}
$$


Using the following equations

$$
E(\hat{\beta}-\beta)(\hat{\beta}-\beta)^{\prime}=\left(X^{\prime} X\right)^{-1} X^{\prime} R X\left(X^{\prime} X\right)^{-1}
$$

and

$$
E(\tilde{\beta}-\beta)(\tilde{\beta}-\beta)^{\prime}=\left(X^{\prime} R^{-1} X\right)^{-1}
$$

we can obtain the covariance matrices of LSE and BLUE, and their differences. To simplify the simulation, we limit the design matrix $X$ to the one dimensional case. When the sample size becomes very large, the difference should be becomes almost negligible. The detailed simulation code and results are provided in Appendix 1.

\subsection{Simulation for Central Limit Theorem}

According to LEMMA 2, asymptotically $T_{n}$ follows a normal distribution, so the estimator of $\beta$ also follows a normal distribution. In this section, we only need to test the normality of $T_{n}$. To simplify the calculation, we only do the one dimensional case for both polynomial regression and trigonometric regression. For LSE, we will use

$$
T_{n}^{\mathrm{LSE}}=\sum_{i=1}^{n} 2 \epsilon_{i} \sum_{n}^{-\frac{1}{2}} X_{i},
$$

and for BLUE we will use

$$
T_{n}^{B L U E}=\sum_{i=1}^{n} 2 g_{i}\left(\Gamma_{n}\right)^{-\frac{1}{2}} B_{i},
$$

where $g=\left\{g_{l}\right\}_{i=1}^{n}$ follows normal distribution with zero-mean and $B_{i}$ is a 
transformation of $x_{i}$ as we stated in section 5.4.

Hence there are four tests to be done:

1. LSE in polynomial regression;

2. LSE in trigonometric regression;

3. BLUE in polynomial regression; and

4. BLUE in trigonometric regression.

For each case, we will generate 500 samples. Using the samples, we will generate a histogram and a QQ plot, and test them with the Shapiro-Wilk and Kolmogorov-Smirnov tests.

The final results show that all of samples from the four test cases follow a normal distribution based on the plots and tests. The detailed simulation code and results can be found in Appendix 2. 


\section{Conclusion}

The problem of asymptotic efficiency of the least square estimator (LSE) relative to the best linear unbiased estimator (BLUE) in linear regression is revealed when we consider dependent errors. In Chapter 4, we studied the Grenander \& Rosenblatt's (1984) approach and discovered that the LSE of $\beta$, the regression coefficients is asymptotically efficient relative to the BLUE, if and only if the spectral density is constant on each of the elements of $S$ (the regression spectrum). But for particular case of design such as polynomial or trigonometric ones, the LSE is always asymptotically efficient regardless of the form of spectral density (provided it is positive and piecewise continuous). It is also worthwhile examining the M-estimation based on Wu's (2007) paper, in order to derive some Bahadur representations and CLT for LSE and BLUE in Chapter 5, and many more estimators useful with infinite variance errors.

The conclusions in Chapter 4 and Chapter 5 are illustrated by a computer simulation. In section 6.1, we simulated the covariance matrices for both LSE and BLUE of $\beta$ in polynomial or trigonometric design cases, and we found that the difference between them was quite small. This result confirmed the conclusion reached in Chapter 4 . Also, in section 6.2, we simulated a large size of $T_{n}$ 's for four different cases. Each of them followed asymptotically a normal distribution. This result was shown by histograms, QQ plots, the Shapiro-Wilk test, and the Kolmogorov-Smirnov test. Hence, the conclusion in 
Chapter 5 is also matched with the computer simulation.

In order to develop the CLT for BLUE, some limitations to our conclusion are in Chapter 5. We need to assume that $g=\left\{g_{l}\right\}_{t=1}^{n}$ follows a normal distribution with zero-mean. Hence, a further study should be made without the normality assumption. 


\section{References}

Ambrosio, L., Fusco, N. \& Pallara, D. (2000) Functions of Bounded Variation and Free

Discontinuity Problems. Oxford Mathematical Monographs. The Clarendon Press, Oxford University Press, New York.

Billingley, P. (1995). Probability and Measure. 3rd Ed. Wiley. New York.

Brockwell, P. J. \& Davis, R. A. (2002). Introduction to Time Series and Forecasting. Springer-Berlag, New York. 15.

Brockwell, P. J. \& Davis, R. A. (1991). Time Series: Theory and Methods. $2^{\text {nd }}$ Ed. Springer-Verlag, New York. 117.

Grenander, U. (1954). On Estimation of Regression Coefficients in the Case of an Autocorrelated Disturbance. Ann. Mathe. Statist. 25, 252-272.

Grenander, U. \& Rosenblatt, M. (1984). Statistical Analysis of Stationary Time Series. Chelsea Publishing Company, New York. 86-87, 226-248.

Halmos, P. R. (1958). Finite Dimensional Vector Spaces. D. Van Nostrand company, INC, New York, 74.

Huber, P. J. (1981). Robust Statistics. Wiley, New York.

Montgomery, D. C., Peck, E. A., and Vining, G. G. (2001). Introduction to Linear Regression Analysis, 3rd Ed. Wiley Series in Probability and Statistics, New York. 604-605. 
Rousseeuw, P. J. \& Leroy, A. M. (2003). Robust Reggression and Outlier Detection. Wiley, New York.

Wu, Wei Biao. (2005). Nonlinear system theory: Another look at dependence. Proc. Natl. Acad. Sci. USA 102 14,150-14,154. MR2172215

Wu, Wei Biao. (2006). M-estimation of linear models with dependent errors. Available at http://arxiv.org/abs/math/0412268.

Wu, Wei Biao. (2007). M-estimation of linear models with dependent errors. Ann. Stat. 35, 495-521. 


\section{Appendix 1: Simulation Code and Result for Section 6.1}

\section{Polynomial Regression}

Simulation code for polynomial regression case:

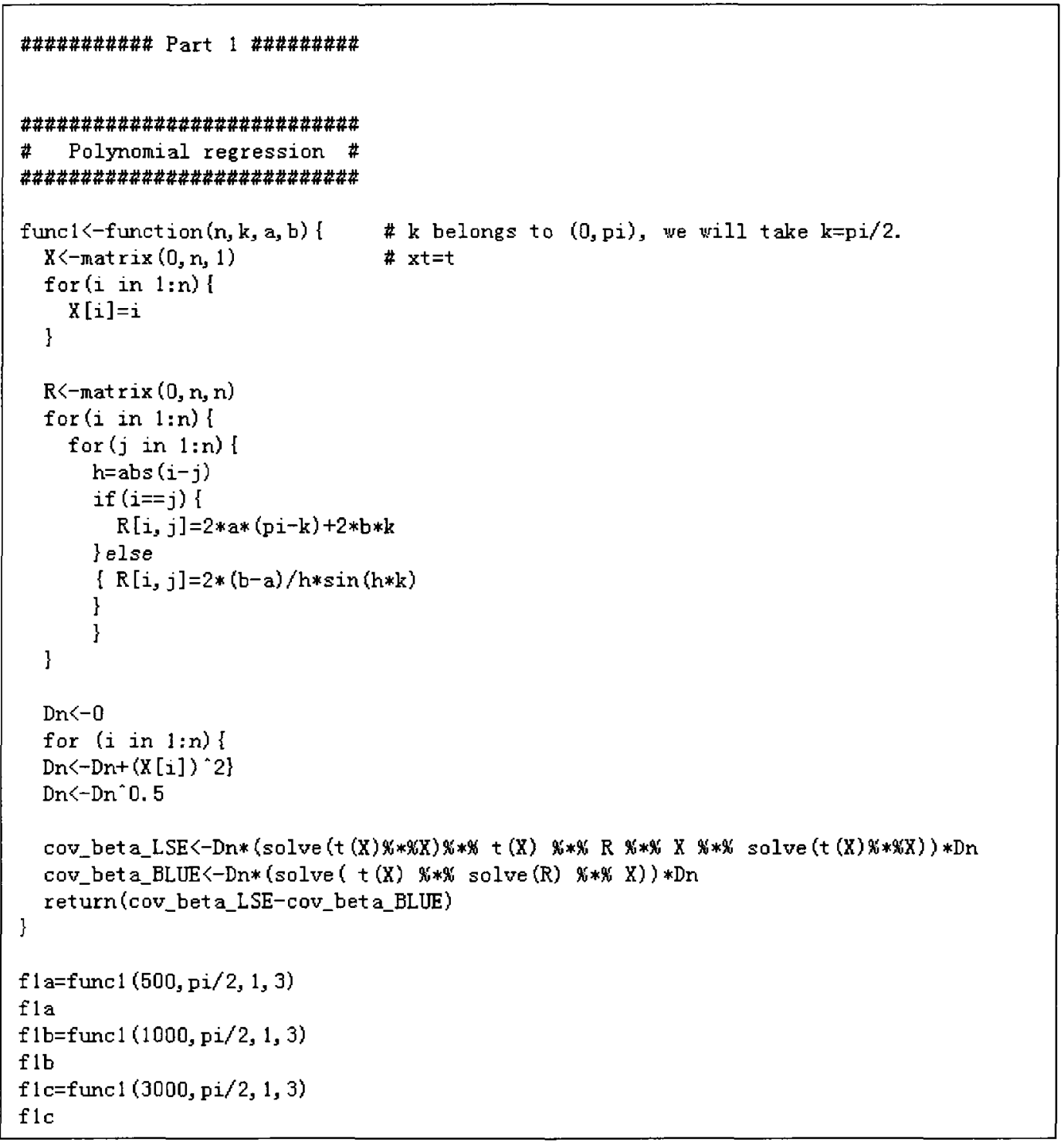


The returned the value is the difference between the covariance of LSE and covariance of BLUE. The following are four test cases with increasing sample size and their results:

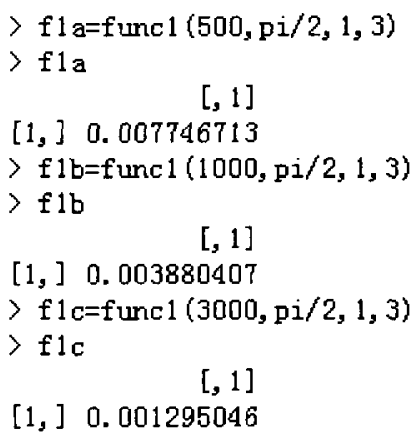

If we take $k=\frac{\pi}{2}, a=1$, and $b=3$, the computer returns the differences as:

\begin{tabular}{|l|l|}
\hline Sample Size (n) & Covariance Matrix Difference between LSE and BLUE \\
\hline 500 & 0.007764713 \\
\hline 1000 & 0.003880407 \\
\hline 3000 & 0.001295046 \\
\hline
\end{tabular}

We can see that as the sample size increases, the differences between the covariance matrices decreases. Hence we can say that the LSE is asymptotically efficient relative to BLUE in those cases. 


\section{Trigonometric Regression}

Simulation code for trigonometric regression case:

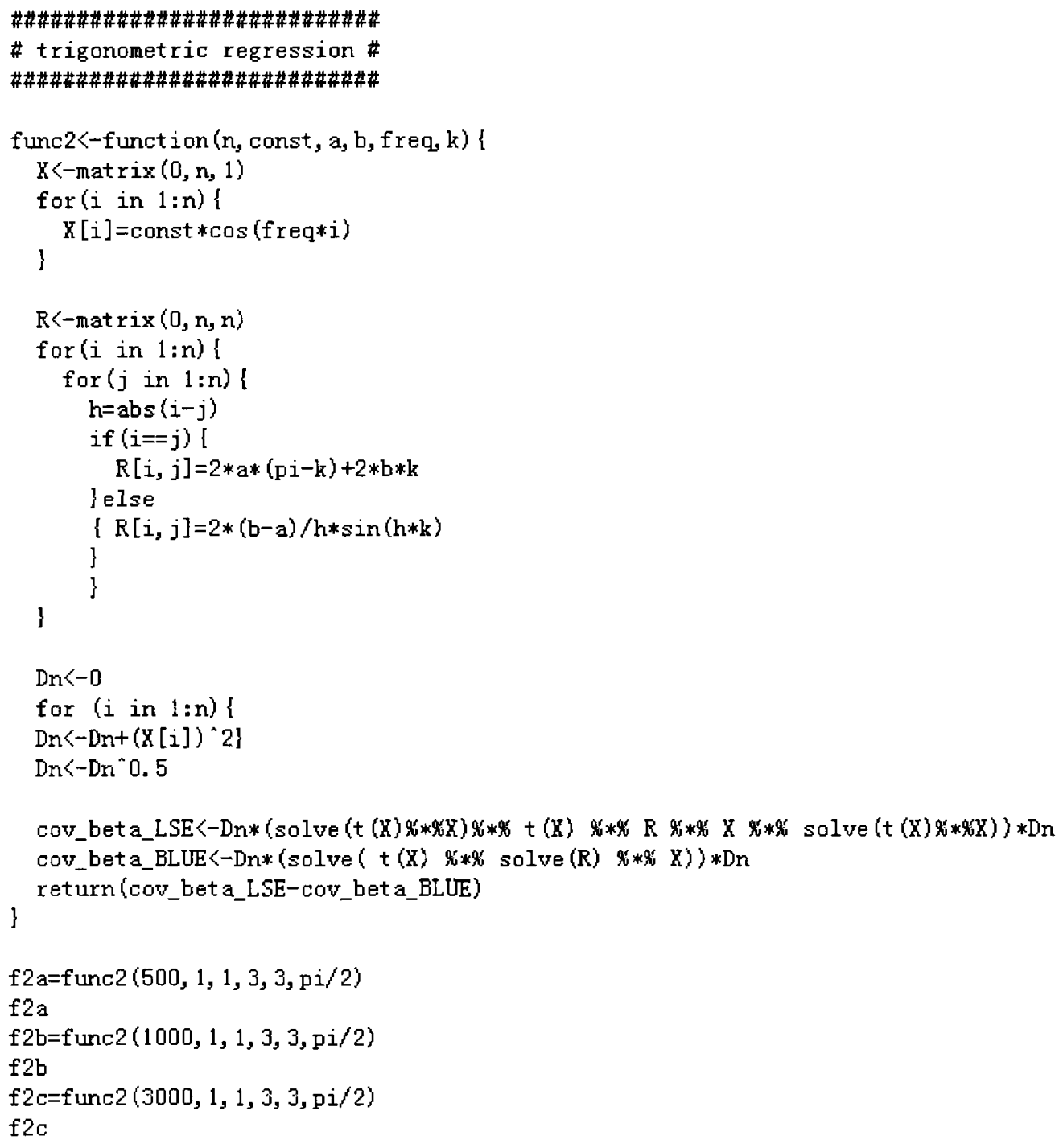

The returned the value is the difference between the covariance of LSE and covariance of BLUE. There are four test cases with different parameters: 
$\mathrm{f} 2 \mathrm{a}=\mathrm{func} 2(500,1,1,3,3, \mathrm{pi} / 2)$

$>\mathrm{f} 2 \mathrm{a}$

$[1]$,

$>\mathrm{f} 2 \mathrm{~b}=$ func $2(1000,1,1,3,3, \mathrm{pi} / 2)$

$>f 2 b$

$>\mathrm{f} 2 \mathrm{c}=\mathrm{func} 2(3000,1,1,3,3, \mathrm{pi} / 2)$

$>f 2 c$

$[1]$,

If we take const $=1, a=1, b=3$, freq $=3, k=\frac{\pi}{2}$ and, the computer returns the differences as:

\begin{tabular}{|l|l|}
\hline Sample Size $(\mathrm{n})$ & Covariance Matrix Difference between LSE and BLUE \\
\hline 500 & 0.00377273 \\
\hline 1000 & 0.003456541 \\
\hline 3000 & 0.0009288768 \\
\hline
\end{tabular}

We can see that as the sample size increases, the differences between the covariance

matrices decreases. Hence we can say that the LSE is asymptotical efficient relative to BLUE in those cases. Note that because of the limitation of software $\mathrm{R}$, the sample size could not be made greater. 


\section{Appendix 2: Simulation Code and Result for Section 6.2}

\section{LSE in Polynomial Regression}

Simulation code for polynomial regression case:

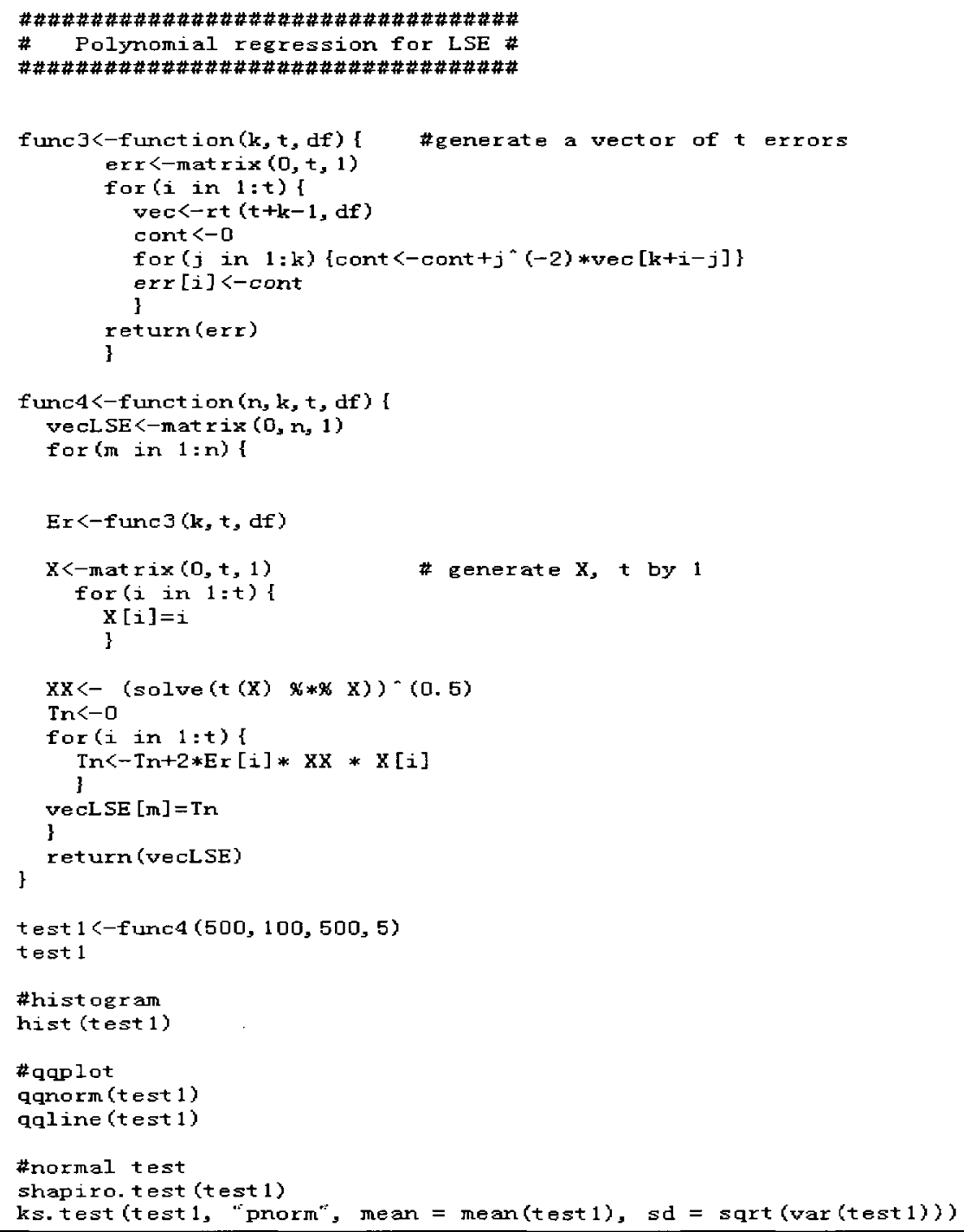


We are going to generate $t=500$ errors follows a moving average process

$$
\epsilon_{t}=\sum_{j=1}^{\infty} \frac{1}{j^{2}} e_{t-j}
$$

where $e_{1}$ follows a student-t distribution with degree of freedom of 5 . Also, we take 100

instead of $\infty$. Then using the 500 errors and a design matrix $\mathrm{X}$ with polynomial regression, we obtain a $T_{n}$. Repeating the above process 500 times, we generate a sample size of 500 . The computer output is shown below:

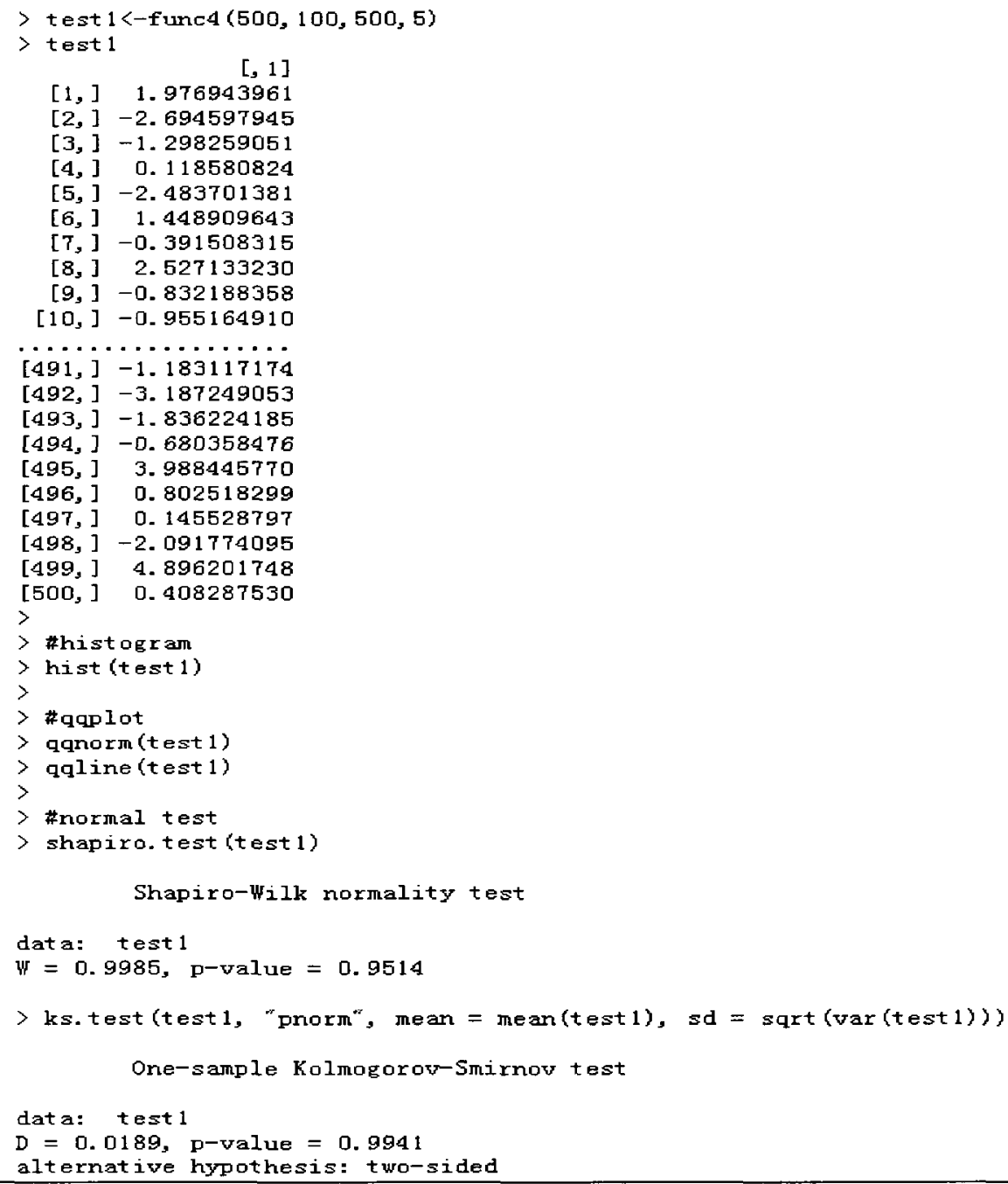


The histogram and QQ plot:

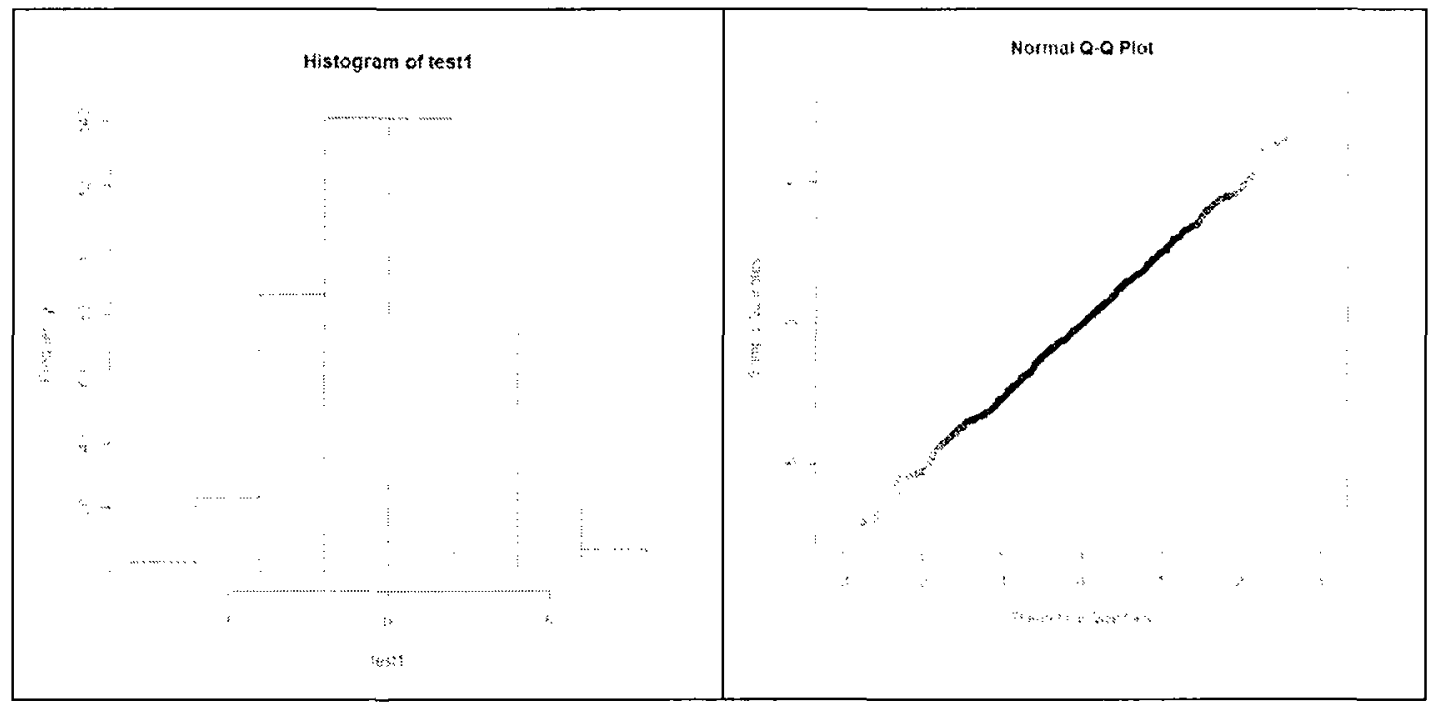

Hypothesis tests:

\begin{tabular}{|l|l|l|}
\hline & Test Statistic & p-value \\
\hline Shapiro-Wilk Normality Test & $\mathrm{W}=0.9985$ & 0.9514 \\
\hline One-sample Kolmogorov-Smirnov Test & $\mathrm{D}=0.0189$ & 0.9941 \\
\hline
\end{tabular}

In all plots and tests, the results show that the large sample LSE follow normal distributions. 


\section{LSE in Trigonometric Regression}

Simulation code for trigonometric regression case:

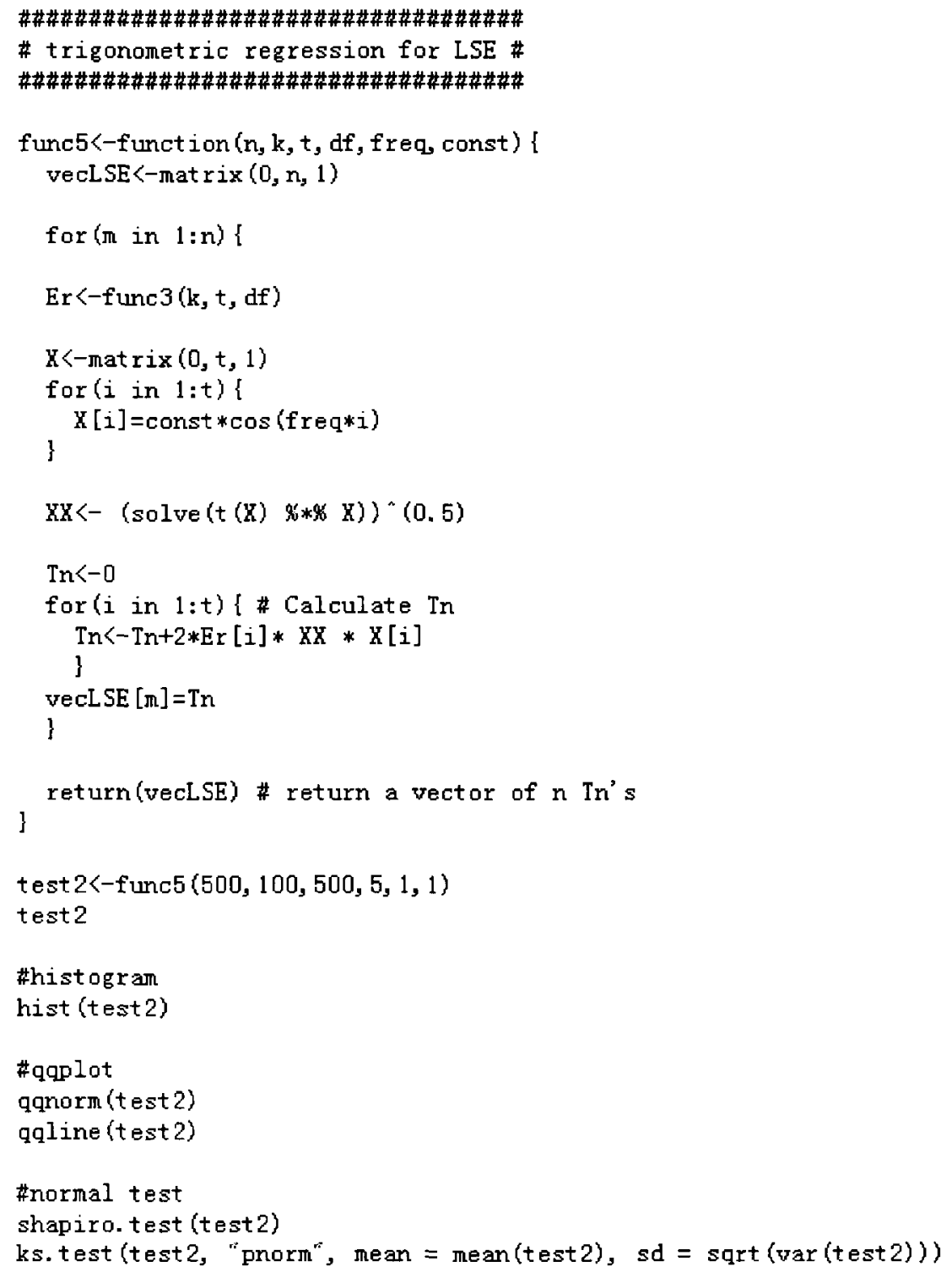

We deploy the same methods as used previously to generate 500 errors. $T_{n}$ can be obtained by the errors, and a design matrix $X$ can be obtained with trigonometric 
regression. Taking a frequency of 1 and constant of 0 , the above process was repeated

500 times. The computer output is shown below:

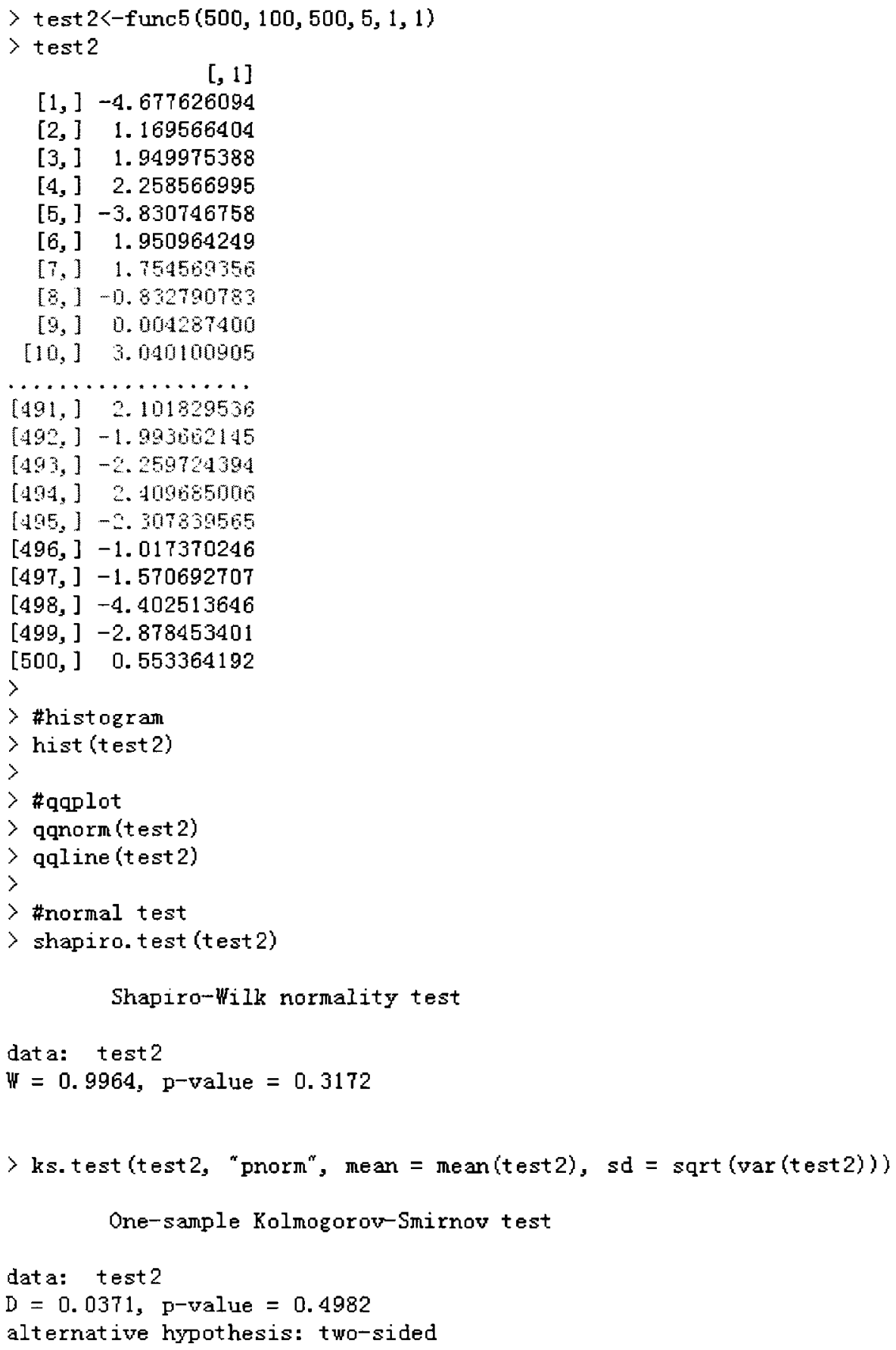


The histogram and QQ plot:

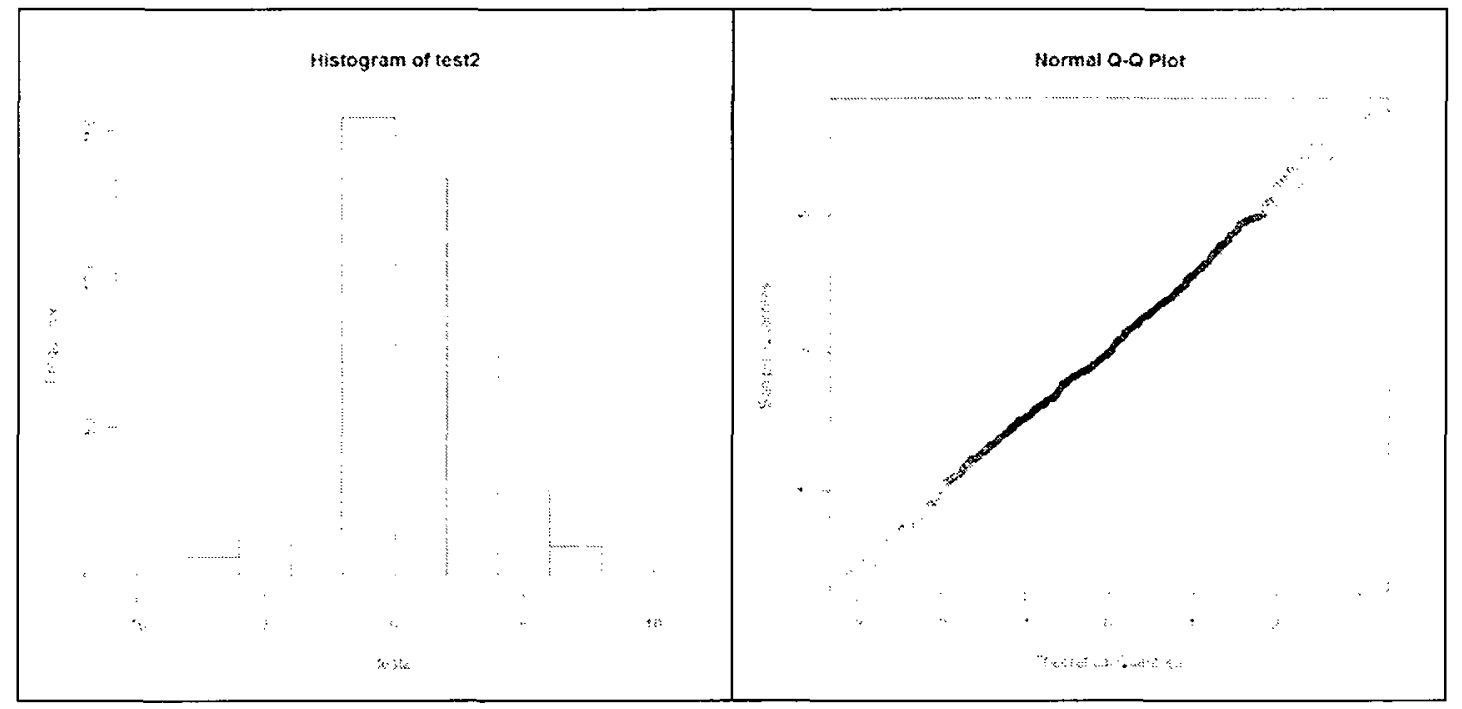

Hypothesis tests:

\begin{tabular}{|l|l|l|}
\hline & Test Statistic & p-value \\
\hline Shapiro-Wilk Normality Test & $\mathrm{W}=0.9964$ & 0.3172 \\
\hline One-sample Kolmogorov-Smirnov Test & $\mathrm{D}=0.0371$ & 0.4982 \\
\hline
\end{tabular}

In all plots and the tests, the results show that the large sample LSE follow normal distributions. 


\section{BLUE in Polynomial Regression}

Simulation code for polynomial regression case:

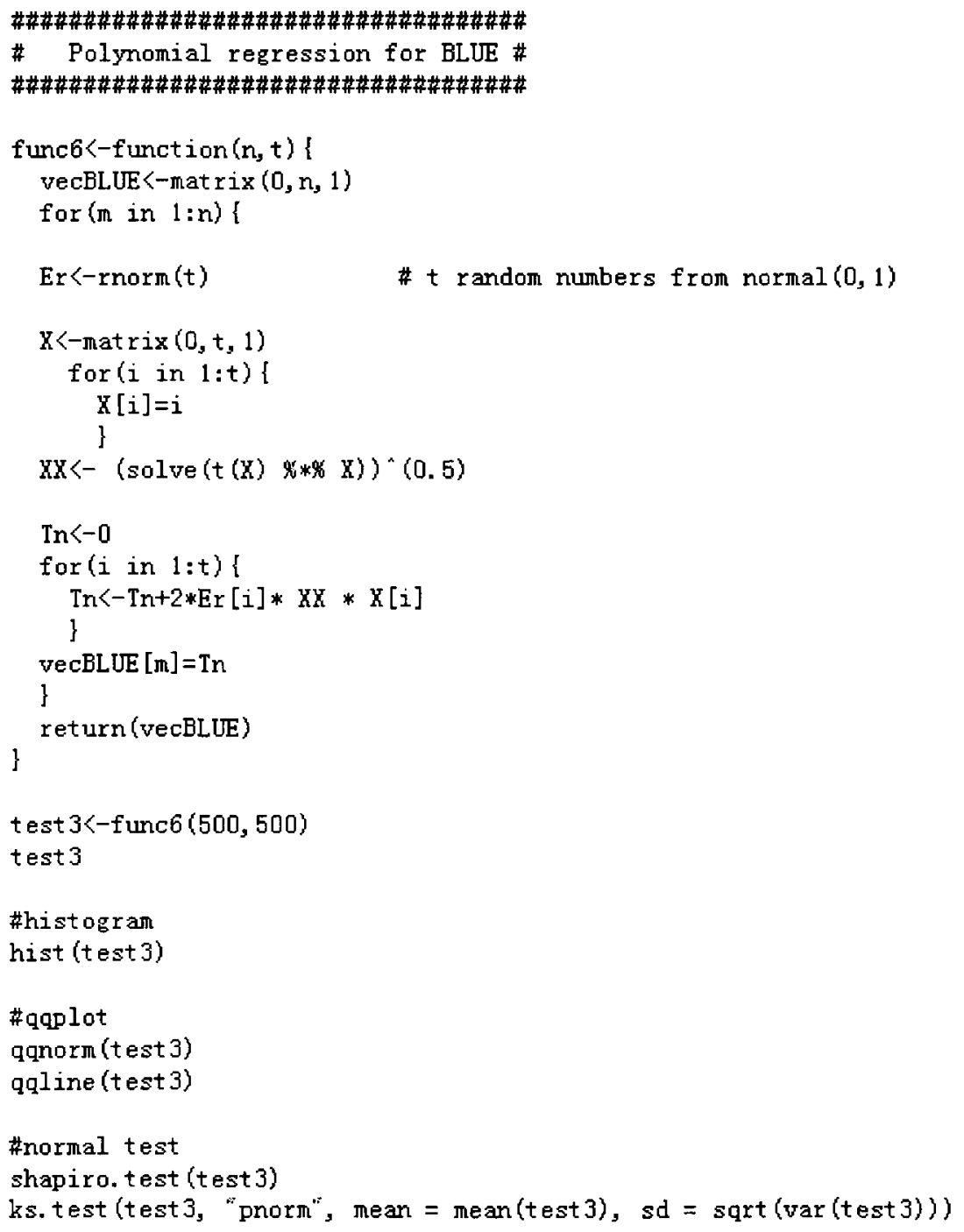

As we mentioned before, $g=\left\{g_{t}\right\}_{t=1}^{n}$ follows a normal distribution with zero-mean, and we can generate a sample of $500 g_{\imath}$ from Normal $(0,1)$. Repeating the process, we obtain $T_{n}$ for 500 times. The computer output is shown below: 


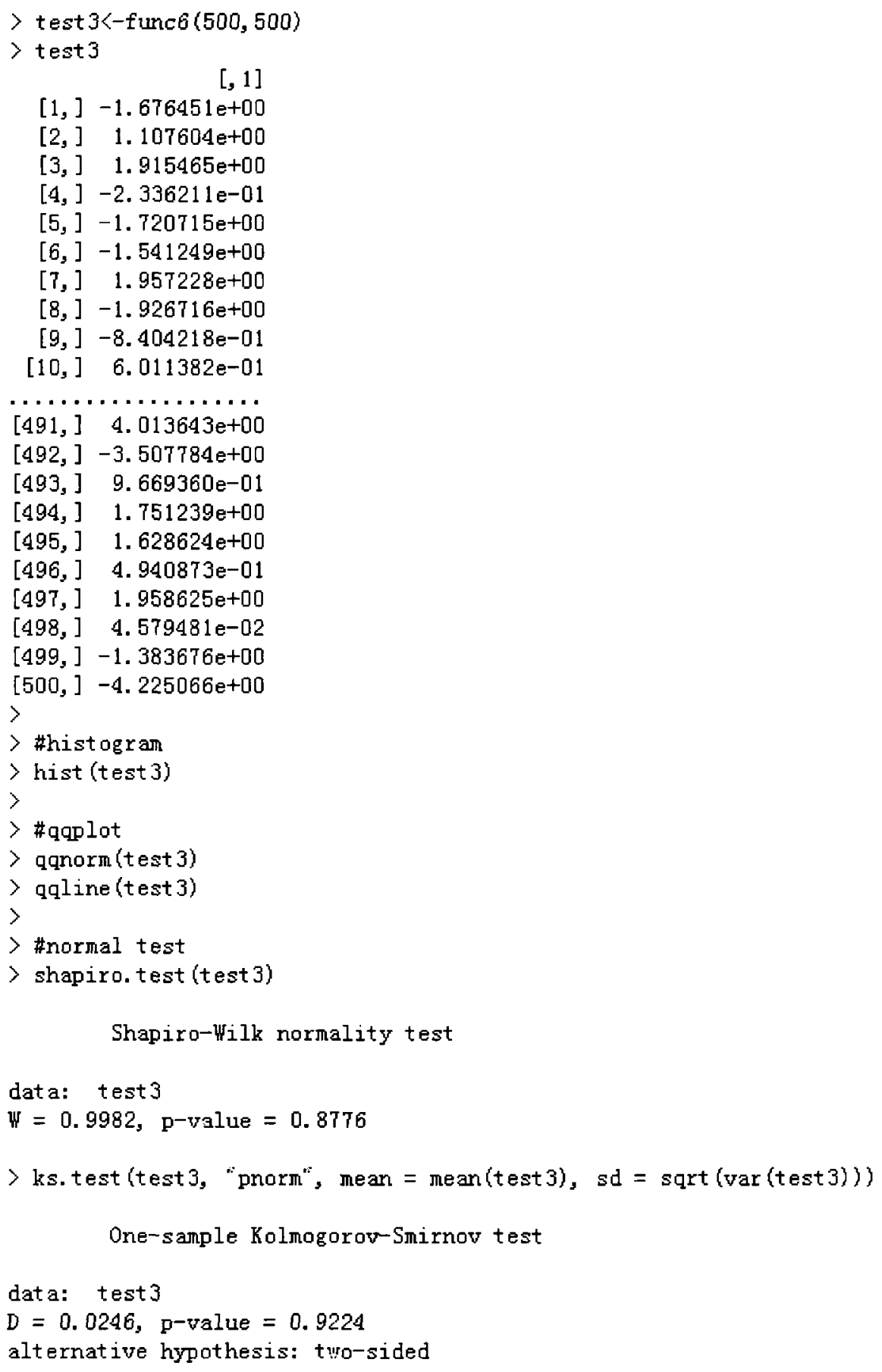

The histogram and QQ plot: 


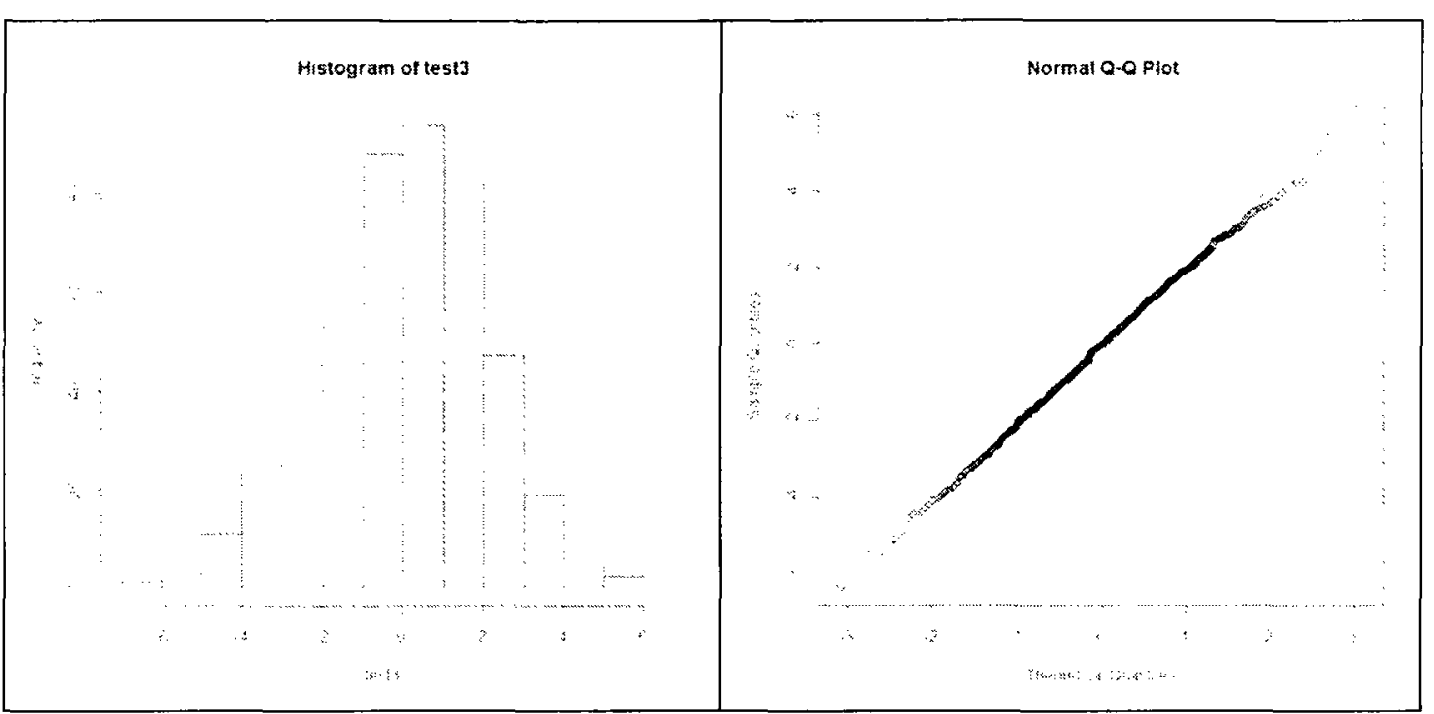

Hypothesis tests:

\begin{tabular}{|l|l|l|}
\hline & Test Statistic & p-value \\
\hline Shapiro-Wilk Normality Test & $\mathrm{W}=0.9982$ & 0.8776 \\
\hline One-sample Kolmogorov-Smirnov Test & $\mathrm{D}=0.0246$ & 0.9224 \\
\hline
\end{tabular}

In all plots and tests, the results show that the large sample BLUE follow normal distributions. 


\section{BLUE in Trigonometric Regression}

Simulation code for trigonometric regression case:

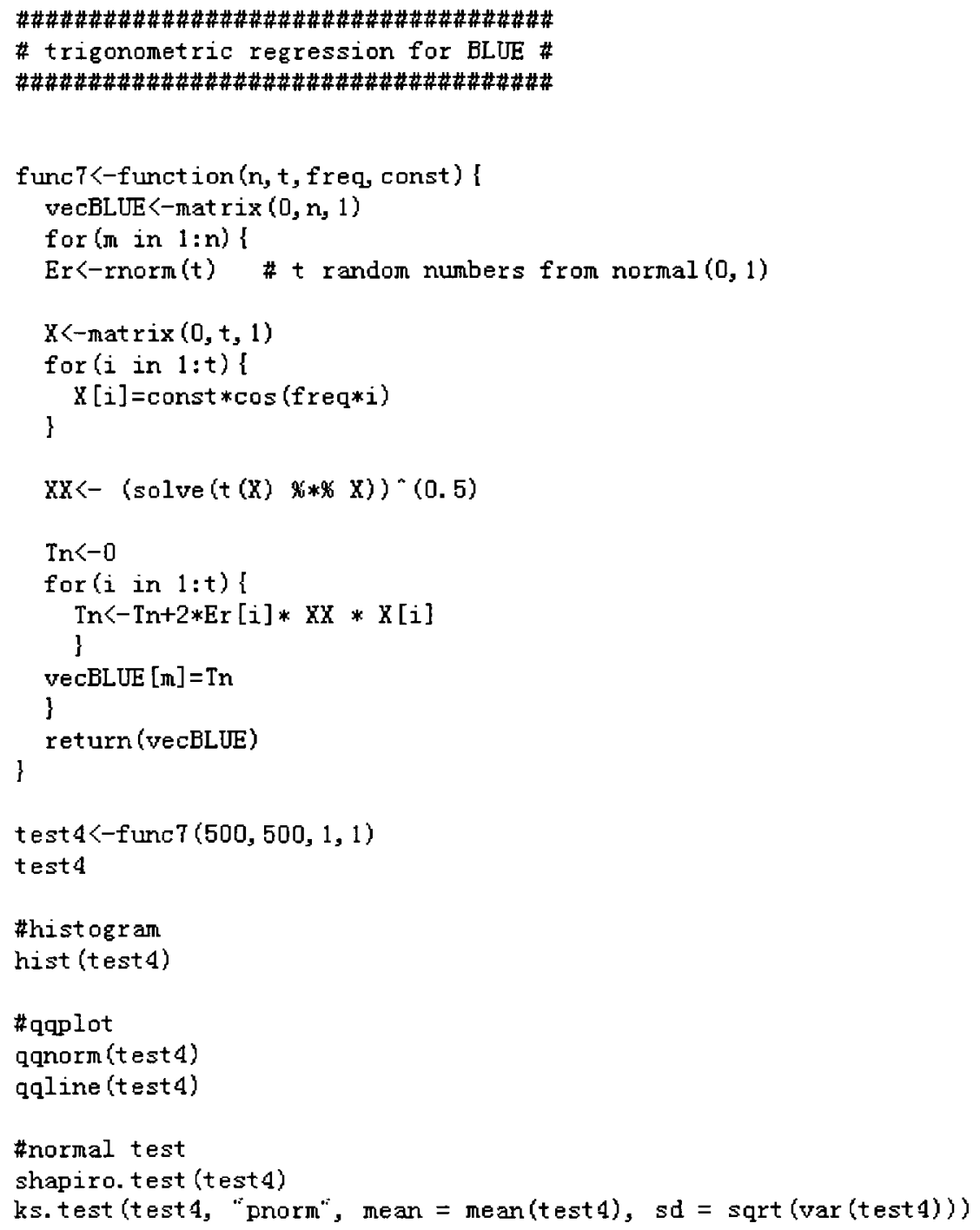

Using Normal $(0,1)$, we generate 500 random errors. $T_{n}$ can be obtained by the errors, and a design matrix $\mathrm{X}$ can be obtained with trigonometric regression. We take a frequency of 1 and constant of 0 . The above process was repeated 500 times. The 
computer output is shown below:

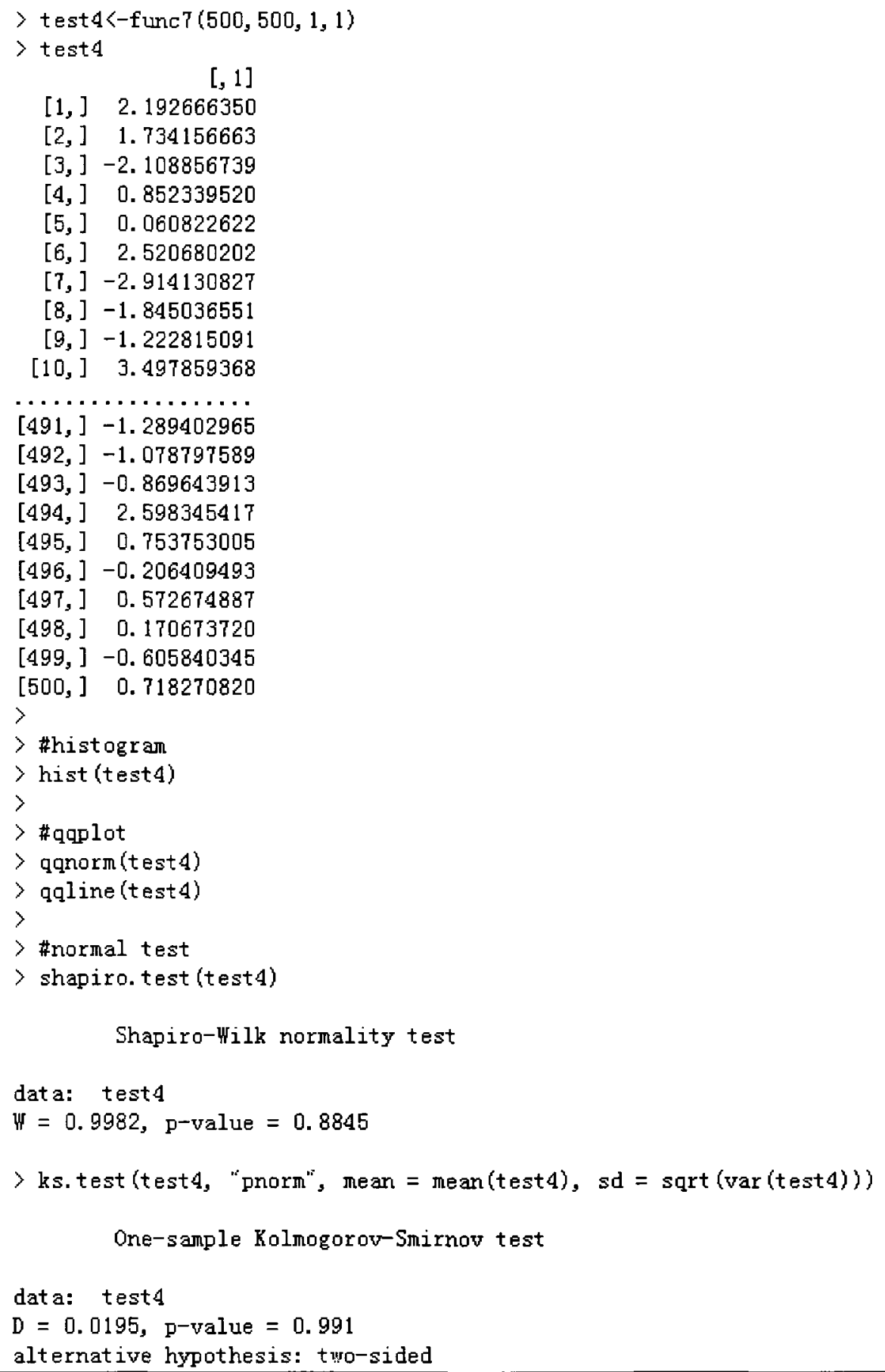

The histogram and QQ plot: 


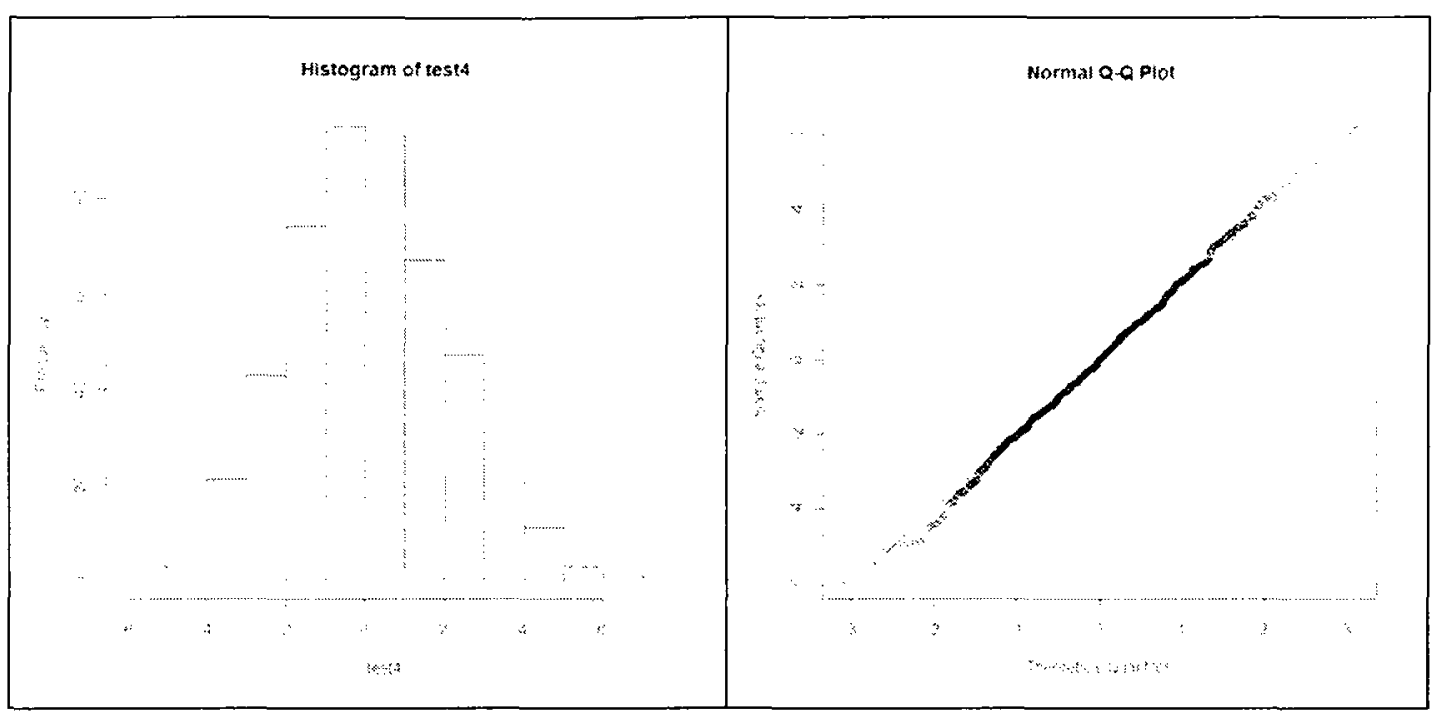

Hypothesis tests:

\begin{tabular}{|l|l|l|}
\hline & Test Statistic & $\mathrm{p}$-value \\
\hline Shapiro-Wilk Normality Test & $\mathrm{W}=0.9982$ & 0.8845 \\
\hline One-sample Kolmogorov-Smirnov Test & $\mathrm{D}=0.0195$ & 0.991 \\
\hline
\end{tabular}

In all plots and tests, the results show that the large sample BLUE follow normal distributions. 